\title{
Driven granular gas with van der Waals interaction
}

\author{
Dissertation \\ for the award of the degree \\ "Doctor rerum naturalium" \\ of the Georg-August-Universität Göttingen
}

within the doctoral program ProPhys

of the Georg-August University School of Science (GAUSS)

submitted by

Qiong Bai

from Shanxi, China

Göttingen, 2018 


\title{
Thesis Committee
}

Prof. Dr. Stephan Herminghaus

Dynamik komplexer Fluide,

Max-Planck-Institut für Dynamik und Selbstorganisation, Göttingen

\section{Prof. Dr. Annette Zippelius}

Institut für Theoretische Physik, Georg-August-Universität Göttingen

\section{Prof. Dr. Marco G. Mazza}

Interdisciplinary Centre for Mathematical Modelling,

Loughborough University, Loughborough, United Kingdom

Dynamik komplexer Fluide,

Max-Planck-Institut für Dynamik und Selbstorganisation, Göttingen

\section{Members of the Examination Board}

\author{
Reviewer: Prof. Dr. Marco G. Mazza
}

Interdisciplinary Centre for Mathematical Modelling,

Loughborough University, Loughborough, United Kingdom

Dynamik komplexer Fluide,

Max-Planck-Institut für Dynamik und Selbstorganisation, Göttingen

\section{Second Reviewer: Prof. Dr. Stephan Herminghaus}

Dynamik komplexer Fluide,

Max-Planck-Institut für Dynamik und Selbstorganisation, Göttingen

\section{Further members of the Examination Board:}

\section{Prof. Dr. Annette Zippelius}

Institut für Theoretische Physik, Georg-August-Universität Göttingen

\section{Prof. Dr. Stefan Klumpp}

Institut für Nichtlineare Dynamik,, Georg-August-Universität Göttingen

\section{Dr. Michael Wilczek}

Theorie turbulenter Strömungen,

Max-Planck-Institut für Dynamik und Selbstorganisation, Göttingen 
Priv.-Doz. Dr. Salvatore R. Manmana

Institut für Theoretische Physik, Georg-August-Universität Göttingen

Date of Oral Examination: September 24th, 2018 


\section{Abstract}

Granular matter is a collection of solid particles which inelastically collide with each other with possible size range from $1 \mu \mathrm{m}$ (like clays) to $1 \mathrm{~km}$ (like some asteroids). Due to the characteristic of dissipation, a myriad of interesting phenomena are observed in granular system, such as pattern formation, "Brazil nut effect", and phase separations. Most of them find a direct connection to our daily life or industrial processes. Additional cohesive forces between grains may significantly change the behavior of the system. For granular particles with sizes smaller than $100 \mu \mathrm{m}$, one adhesive interaction, the van der Waals force, starts to play a role. It has been recognized that van der Waals interaction plays an important role in the generation of asteroids. However, detailed explorations of the influence by van der Waals interactions on granular materials are still lacking.

In this thesis we try to fill this gap and numerically study how van der Waals interactions affect the collective behavior of excited granular gas. We perform time-driven molecular dynamics simulations. The system we simulate is composed of monodisperse spherical particles with a diameter of $70 \mu \mathrm{m}$, which are confined by two parallel walls separated by a fixed distance. The restitution coefficient $\epsilon$ quantifies the dissipation, and the Hamaker constant sets the strength of the van der Waals interaction. The whole system is vibrated sinusoidally in the direction perpendicular to the walls, and the particles gain energy via collisions with the two walls. Particles will feel conservative attractive forces when they approach each other (modeled by the Hamaker theory) and inelastically collide with each other when they touch (modeled by the spring-dashpot model).

We map the phase diagram of shaking amplitude $A$ and average filling fraction $\bar{\phi}$, and a new solid-like and gas coexistence regime is found, together with cluster and homogeneous states. We characterize the different granular phases by means of a set of order parameters, such as bond orientational order parameter, coordination number, and connection number. The solid-like part of the coexistence part could transform from a random close packing state (with local filling fraction 0.64) to a poly-crystalline state (with hcp or fcc local structures) with $A$ increasing. The boundary line of the transition can be modulated by the restitution coefficient $\epsilon$ and the Hamaker constant, but is independent of $\bar{\phi}$. We show that the transition is due to different quenching speeds, a combined effect of the energy dissipation during collisions and energy injection through the walls. 



\section{Contents}

$\begin{array}{lll}1 & \text { Introduction } & 1\end{array}$

1.1 What is granular matter? . . . . . . . . . . . . . . . . . . 1

1.2 Classical questions in granular system . . . . . . . . . . . . . . . 2

$1.2 .1 \quad$ Angle of repose . . . . . . . . . . . . . . . 2

1.2 .2 Segregation . . . . . . . . . . . . . . . 3

1.3 Granular systems as models . . . . . . . . . . . . . . . . . . 5

1.4 Current research points of interest . . . . . . . . . . . . . . . 5

1.4.1 Granular system with non-spherical shapes . . . . . . . . . . . . 6

1.4 .2 Methods to excit granular system. . . . . . . . . . . . . 8

1.4 .3 Phase transitions in granular system . . . . . . . . . . . . 8

1.4 .4 Phase separations in granular system . . . . . . . . . . . . . . . 9

1.4 .5 Granular system with extra interactions . . . . . . . . . . . . . 10

1.5 Research methods . . . . . . . . . . . . . . . . . . . . 11

1.5 .1 Theoretical . . . . . . . . . . . . . . . 11

1.5 .2 Experimental . . . . . . . . . . . . . . . . . 12

1.5 .3 Numerical . . . . . . . . . . . . . . . . . . . 12

1.6 Aims and scope of the thesis . . . . . . . . . . . . . . . . 13

1.6 .1 Aims . . . . . . . . . . . . . . . . . . . . 13

1.6 .2 Scope $\ldots \ldots \ldots \ldots \ldots \ldots$

2 Theoretical and computational methods $\quad 17$

$2.1 \quad$ Governing equations . . . . . . . . . . . . . . . . . . . . . . . 18

2.2 The force models $\ldots \ldots \ldots \ldots$

$2.2 .1 \quad$ Particle-particle contact force . . . . . . . . . . . . . . . . 19

$2.2 .2 \quad$ Particle-particle non-contact forces . . . . . . . . . . . . . . 21

2.2 .3 The particle-wall forces . . . . . . . . . . . . . . . . 23

2.3 Structure of the simulation algorithm . . . . . . . . . . . . . . . . . . . 24

2.3 .1 The particle propagation . . . . . . . . . . . 26 
2.3 .2 The neighbor list . . . . . . . . . . . . . . . . 27

2.4 Choice of parameters . . . . . . . . . . . . . . . . . . . 31

2.5 Van der Waals force shift $\ldots \ldots \ldots \ldots$. . . . . . . . . . . . . . 32

2.6 Observables . . . . . . . . . . . . . . . . . . . . . . . . . . . . . . . . . . . . 33

2.6 .1 The radial distribution function $g(r) \ldots \ldots . \ldots . \ldots . . \ldots 33$

2.6 .2 The coordination number $Z$. . . . . . . . . . . 34

\begin{tabular}{|lll}
\hline 2.6 .3 & The bond orientational order parameters: local $q_{6}$ and global $Q_{6}$ & 34
\end{tabular}

2.6 .4 The connection number $\xi \ldots \ldots \ldots$. . . . . . . . . . 37

2.7 Conclusion $\ldots \ldots \ldots \ldots \ldots$

3 System with vdW interaction $\quad 41$

3.1 Collisions between two particles with van der Waals interactions . . . . . 41

3.2 The system without van der Waals interaction . . . . . . . . . . . . 41

3.3 Theoretical prediction $\ldots \ldots \ldots \ldots \ldots$

3.4 Simulation details $\ldots \ldots \ldots$. . . . . . . . . . . . . . . . . . 44

$3.4 .1 \quad$ Energy convergence . . . . . . . . . . . . . . . . . 44

3.4 .2 Hysteresis . . . . . . . . . . . . . . . . . . . . . 45

3.4 .3 Stabilization of the phases . . . . . . . . . . . . . 45

3.5 Conclusion . . . . . . . . . . . . . . . . . . . . . . . . . . 46

4 Phase diagram 53

4.1 Phase diagram with Snapshots . . . . . . . . . . . . . . . . . 53

4.2 Characterization of phases . . . . . . . . . . . . . . . . . 55

4.2 .1 Local filling fraction . . . . . . . . . . . . . . . 55

$4.2 .2 \mathrm{RDF} \ldots \ldots \ldots \ldots \ldots \ldots$

$4.2 .3 \quad q_{6}$ and coordination number $Z$. . . . . . . . . . . . . . . 58

4.2 .4 Connection number $\xi \ldots \ldots \ldots \ldots \ldots$

4.3 Conclusion . . . . . . . . . . . . . . . . . . . . . . . . 63

$\begin{array}{lll}5 & \text { Dynamics } & 65\end{array}$

5.1 The time evolution of coexistence phases . . . . . . . . . . . . . . 65

5.2 Crystal structure growth . . . . . . . . . . . . . . . . . 67

5.3 The stability of the crystal structure . . . . . . . . . . . . . . . . . 69

$5.3 .1 \quad$ Irreversibility . . . . . . . . . . . . . . . . 69

5.3 .2 Influence of gravity . . . . . . . . . . . . . . . 71

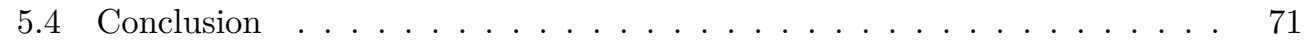


$\begin{array}{lll}6 & \text { Coexistence regions } & 75\end{array}$

$6.1 \quad$ Influences of dissipation . . . . . . . . . . . . . . . . . . 75

6.2 The influence by the van der Waals interaction strength . . . . . . . . . . 78

$\begin{array}{lll}7 & \text { Conclusion } & 81\end{array}$

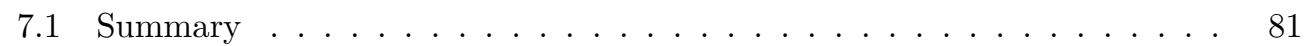

7.2 Discussion and outlook . . . . . . . . . . . . . . . . . . . . 83

7.2 .1 Polydispersity . . . . . . . . . . . . . . . . . 83

7.2 .2 Frequency influence . . . . . . . . . . . . . . . 83

\begin{tabular}{ll}
\hline Bibliography & 85
\end{tabular}

\begin{tabular}{ll}
\hline Acknowlegements & 95
\end{tabular} 


\section{Introduction}

\subsection{What is granular matter?}

A granular material is a collection of discrete solid, macroscopic particles. The characteristics of granular matter is that energy is dissipated during particles' collisions.

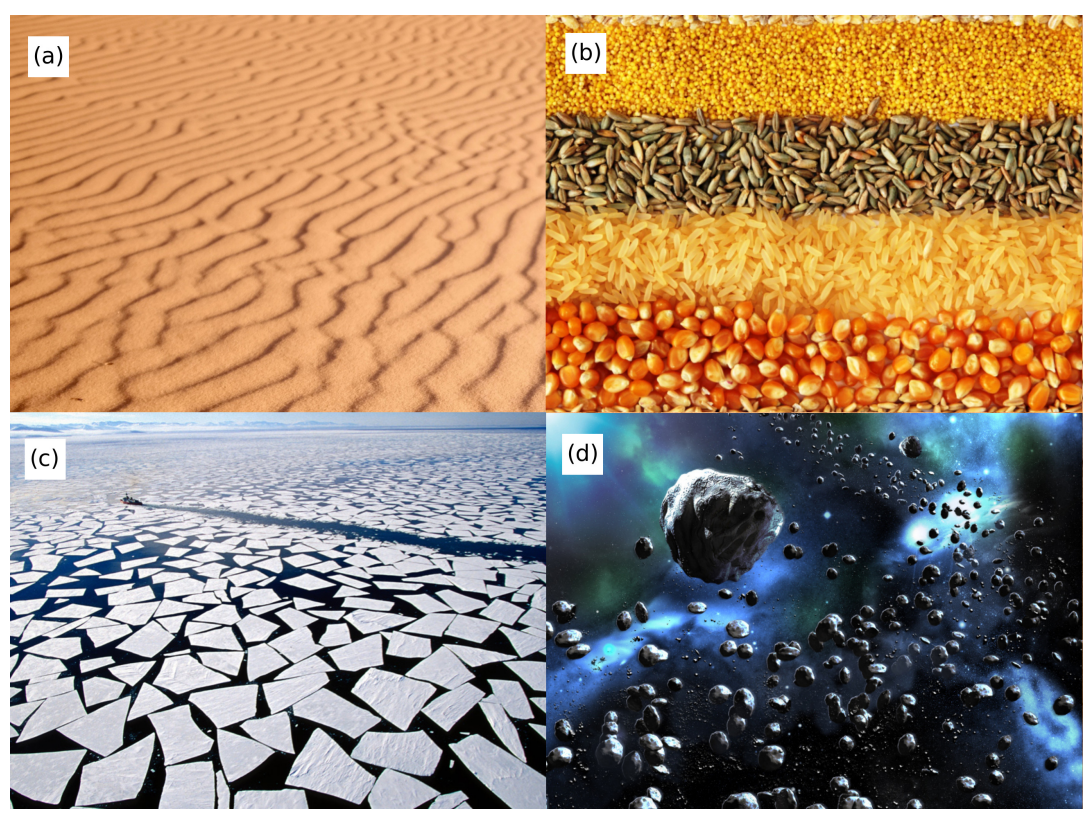

Figure 1.1: The examples of granular matters. (a) sands ${ }^{1}$, (b) grains ${ }^{2}$, (c) icebergs ${ }^{3}$, (d) asteroids $^{4}$.

As shown in Fig. 1.1, the grains in a granular material could be sand, our daily food including rice, coffee beans and pastas, or, at larger scales, icebergs in ice floes and asteroids in the solar system. Granular matter is not a phase of matter. Its motion can be described as gas-like, liquid-like or solid-like depending on the averaged kinetic energy 
of the particles; granular matter exhibits its own unique properties in each of these states which are different from the traditional ones for atomic or molecular systems.

Plant seeds can float in the wind as gas-like particles, and finally rest in random places where new plants will grow. Industrial dust floats very easily and frequently causes smog and air pollution in London, New York in 1950s, or Beijing nowadays. Sand can flow like a liquid in the hourglass. On the other hand, mud-and-stone flow can take one's life away in seconds. Granular materials are handled every day in the pharmaceutical industry, agriculture, and mining industry. It has been estimated that about $10 \%$ of the global energy production is used to to keep and transport raw materials in granular form. Granular matter is thus a field worth understanding more for both its practical applications, but also because it is an ideal system to study generalizations of statistical mechanics to nonequilibrium settings.

\subsection{Classical questions in granular system}

\subsubsection{Angle of repose}

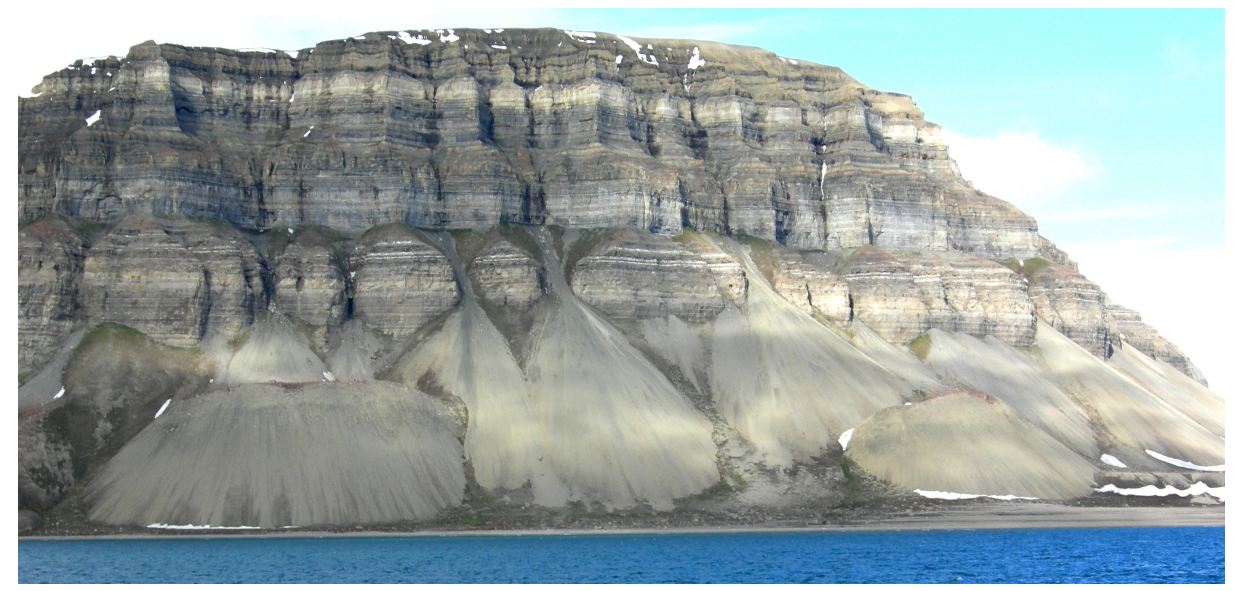

Figure 1.2: Talus cones on north shore of Isfjord, Svalbard, Norway, showing angle of repose for coarse sediment ${ }^{1}$.

When we pour a bucket of grains onto a horizontal surface, a pile will form. The steepest inner angle of the pile relative to the horizontal plane without sliding is the angle of repose, or critical angle of repose $\theta_{c}$. In Fig. 1.2, we show an example the angle of repose generated by nature. $\theta_{c}$ can range from $0^{\circ}$ to $90^{\circ}$. It can be affected by the morphology 
of the granular particles and additional solvents. The study of the angle of repose is very important. For example, it can be used to design the equipment to process and install the granular material. It can also be used to estimate if a slope will collapse or not. One well-known case are the avalanches in mountainous areas.

A question that attracts interest about the angle of repose is: what would happen when the inner angle between the pile surface and horizontal surface $\theta$ exceed $\theta_{c}$. Such configuration can be implemented, for example, if we tilt the container of a sand pile which is at rest with the angle of repose. It turns out that the pile can still be stable as long as $\theta<\theta_{\max }$, where $\theta_{\max }$ is the maximal stable slope angle. When the slope is increased above $\theta_{\max }$, particles will start to slide and an avalanche may occur till $\theta$ decrease to the angle of repose $\theta_{c}(\mathbb{1})$. Experiments, theoretical predictions and numerical calculations have improved our understanding of $\theta_{\max }$ and $\theta_{c}(2,3,4)$. However, to date no simple way has been figured out to calculate these two angles.

Another important issue deals with the relaxation of granular piles by means of perturbations. A granular pile is usually stable when the slope is smaller than the angle of repose. However, it is still a metastable state, and the truly stable state must have a horizontal surface in the field of gravity. One pile can be relaxed by perturbations till it reaches the true stable state. Recently, D. Tsuji et al.(5) performed experiments on a vertically vibrated pile and provided a nonlinear relaxation dynamics theory to explain the observed relaxing process. The actual relaxation was successfully reproduced by the continuity equations. They applied this relaxation dynamics model to the crater relaxation process caused by impact-induced seismic shaking. The maximum crater diameter erased by the shaking they estimated is comparable with the actual grain size. However, at present this relaxation dynamics model can be applied only in a restricted range and to estimate the relaxation time more precise discusses and computations are needed.

\subsubsection{Segregation}

In nature and also in industrial application equally-sized grains are nearly non-existing. Instead, mixtures of similar grains of different sizes are extremely common. Another particular property of mixtures of granular media is their response to stirring or jostling. Unlike a system of two miscible liquids, which can mix well with each other and can form a homogeneous solution, a granular mixture system may separate according to particle size when is shaken or stirred. This kind of demixing phenomena are generally referred to as segregation $(7,8,9)$. When a can containing one large ball and a number of smaller 

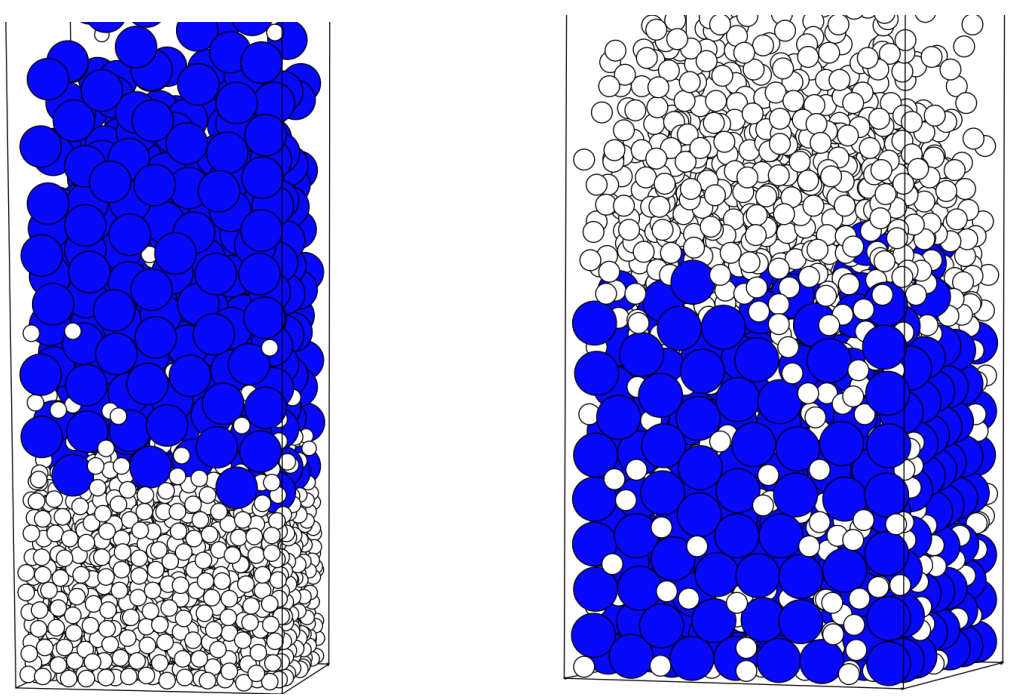

Figure 1.3: The diagrammatic of the Brazil-nut effect (left) and the reverse Brazil-nut effect (right) in mixtures of granular particles of different sizes. Reprinted from (6).

ones is shaken, the large ball may rise to the top. This is the well known "Brazil-nut effect" (10; 11), in which the smaller particles infiltrate into the voids created underneath larger ones.

In 1998, T. Shinbrot et al. (12) observed that the size segregation could happen in reverse. They performed experiments with deep beds filled with fine spheres and two larger intruders; they applied rather large amplitude vibrations, with the typical amplitudes encountered during transportation of solid mixtures in industrial processes. The two intruders have exactly the same size, shape and material but differ in weight. They found that the heavy intruder rises to the top of the bed, while the lighter intruder sinks to the bottom. They explained that inertia causes the heavier intruder to lift off the bed, permitting smaller particles to infiltrate beneath; the lighter intruder could move more easily, preventing the infiltration. In Fig. 1.3, we show two images illustrating the Brazil-nut effect and the reverse Brazil-nut effect from (무).

Segregation is a very complicated process, and it can be effected in counterintuitive ways by the size, shape, density, mixture ratios, applied driven and so on. It can also happen in the lateral direction in a $2 \mathrm{D}$ system (13, 14) and one concrete example is the size sorting between the small pebbles and larger boulders on the surface of the asteroid Itokawa (15). A number of mechanisms have been proposed to explain different segregation phenomena (16; 17), however, more inquiries are necessary. Understanding 
segregation is very important, especially for the material separation and the challenge of mixing powders in industrial processes.

\subsection{Granular systems as models}

The abundant, complex phenomena and properties of granular systems have considerable relevance also to other areas of physics where they are used as models of specific features. A notable example is the work of Bernal (18) who in 1959 carried out his pioneering work and experimentally investigated granular packing with the goal of modeling liquid structures. In 1966, De Gennes (19) used sand pile avalanches as a macroscopic picture for the motion of flux lines in superconductors. In 1990, Clauss et al. (20) found that the nonlinear dynamical phenomena observed in granular system are relevant to the breakdown in semiconductors. The heterogeneous distribution of inter-particle forces within the granular material, known as force chains, generate a complex force chain network (see Fig. 1.4 (21), where each particle is considered as a node. Understanding the force chain in granular system could open a window for achieving a better understanding of complex networks. In additionally, the self-organization paradigm(22, 23, 24) and the slow relaxation present in granular dynamics are considered to have a broad applicability to a host of natural phenomena.

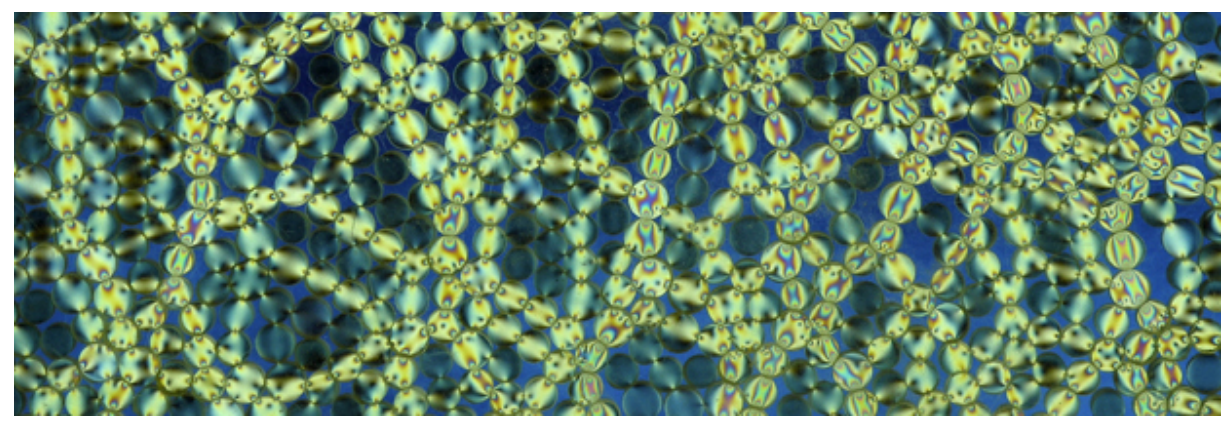

Figure 1.4: The force chains generated in a 2 dimension system. Reprinted from (21).

\subsection{Current research points of interest}

While a number of granular phenomena have been explained and even applied, an abundant number are still lacking explanations and thorough investigations. Reviewing all 
recent work on granular systems is prohibitive, so here we only select some recent representative works and give a short and simple review.

\subsubsection{Granular system with non-spherical shapes}

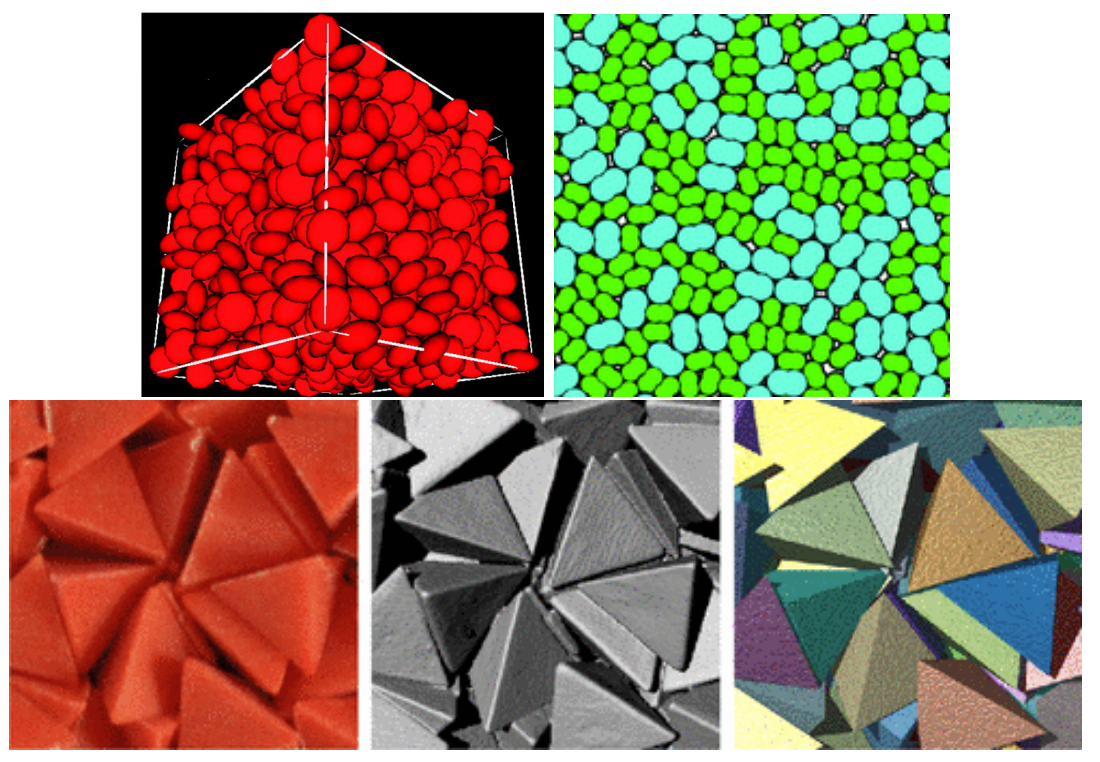

Figure 1.5: The packing for granular particles with special shapes. Top left: The prolate ellipsoid; Top right: the 2D dimer; Bottom: tetrahedra. Reprinted from (25, 26, 27), .

Plenty of work on granular materials, experimental or theoretically, are about systems with spherical particles in 3D or disk in 2D. However, most of real world granular particles have different shapes than rotational invariant shapes like a sphere or a disk. Crops in agriculture, pills and capsules in pharmaceutics usually have elongated or flattened shapes. It have been observed that the particle shape in granular media significantly affects their collective behaviors $(28,29,30)$. The study of packing made of particles with particular shape, which is important for the industries involved in granular processing, emerged in the 1940s. Since then, scientists found that non-spherical shapes can generally achieve larger maximal packing densities than spheres, due to their complicated contact ways. Simulations have found that the densest random packing fractions are at $\phi \approx 0.735$ for prolate ellipsoids (26), at $\phi \approx 0.885$ for $2 \mathrm{D}$ dimers (26), and at $\phi \approx 0.7858$ for tetrahedra (27). Examples for these packing structures are shown in Fig. 1.5 One exception to these very efficient packings are the spaghetti-like thin rods, which pack randomly much 
worse than spheres. A generic theoretical investigation of this field needs to address the complex issue of searching over the very large parameter space of object shapes.

Besides the direct studies on granular materials, particles with particular shape could be used as discrete models to explain physical processes related to other systems. One recent work reported by K. Asencio et al. (31) found a final state of cubic particles resembling the phases observed in colloids. In their experiments, particles were poured in a cylindrical container and the whole system was excited by twists. After a number of sufficiently large intensive twists, a densest final state compatible with the boundary conditions was reached: a series of concentric rings stacked in horizontal layers. Another example is the work recently reported by B. Kou et al. (32). They found both correlation and anti-correlation between different types of clusters in a granular system, which was recognized as a possible approach to learn more about the relaxation dynamics in a system with glassy dynamics. The system they experimentally studied was a 3D hard ellipsoid granular packing driven by oscillatory shear. The elliptic grains had an aspect ratio of 1.5 , a shape that makes crystallization difficult in the system and allows for strong coupling of translational and rotational degrees of freedom.

Recently, B. Nadller et al. (33) developed a purely kinematic continuum model to predict the averaged particle orientation and ordering based on the velocity field and the particle shape. They use a second order orientational tensor $\mathbf{A}$ to describe statistically the particle's orientation at a given points of space and time. The tensor $\mathbf{A}$ has two invariants based on its eigenvalues. One is named as $\zeta$, representing the degree of particle alignment, and the other one is $\alpha$, the misalignment angle. The gradient of velocity field is split into two parts. The symmetrical part $\mathbf{D}$ captures the rate of deformation, and the anti-symmetrical part $\mathbf{W}$ describes the vorticity. A model for $\mathbf{A}$ includes $\mathbf{D}$ and $\mathbf{W}$. There are only two parameters in the model: $\lambda$ which governs the tendency to align with the rate of deformation $D$, and $\Psi$ which is the relaxation parameter. For simplicity, both of them are assumed to be only function of the shape ratio. Nadller et al. (33) compare the results from the model with discrete element method simulations for a simple steady state system under shear and the parameters in the model are set up by comparison with the simulations. They found that the two-parameter model is sufficient to obtain the full orientational tensor $\mathbf{A}$ and capture the essential transient features, which is in good agreement with the simulations. The model provides a foundation to further development of stress response model of the orientational tensor $\mathbf{A}$ for granular materials, and generalize to other flow field. This treatment is not limited to granular system, it can be also adopted to the study of, e.g., biological cells, and polymers. 


\subsubsection{Methods to excit granular system}

Because of the dissipative nature of the granular collisions, after sufficient time, a granular system without any external stimulation will reach a steady state where all particles are motionless. In order to study a granular material, scientists usually excite the system in different ways, such as vibrating (34), shearing (35), rotating (36) and so on. A variety of phenomena take place in the driven granular system, such as pattern formation, convective flows, heaping instabilities, front propagation and so on (37, 38, 39).

Recently, A. K. Mukhopadhyay et al. (40) proposed a new scheme, using a periodically shaken two-dimensional dissipative lattice to drive a multi-species system. The lattice is defined by a periodic potential

$$
V(x, y)=V \cos k_{x} x\left(1+\cos k_{y} y\right)
$$

which is driven via external biharmonic forces

$$
f_{x, y}(t)=d_{x, y}[\cos \omega t+0.25 \cos (2 \omega t+\pi / 2)]
$$

acting in the $x, y$ directions. As the space parity and time-reversal symmetry are broken, particle transport is possible. They found that one system driven by this lattice could separate in a five-dimensional phase space $\left(x, y, p_{x}, p_{y}, t\right)$. The separation depends on the physical characteristics of the species in the mixtures, such as mass, size, shape and so on. The mechanism suggested in (40) is based on a ideal periodic potential field. According to their experience, localized perturbations and weak long-range interactions may improve the segregation efficiency. This segregation mechanism could be applied to the separation of multi-species mixture with particle ranging from atoms to granular grains. More importantly, they supplied a new method to inject energy into the system rather than walls, or any other regular containers. A granular system in other periodic potential field could also show complex phenomena.

\subsubsection{Phase transitions in granular system}

The transition between the three classical regimes (gas-like, liquid-like and solid-like) are driven by the change of density and external forces. In reality, mechanical vibrations can induce transitions in granular systems; this fact is particularly related to phenomena such as the common debris flow or avalanches after earthquakes. Plenty of works have been 
devoted to understand the influence by vibration on the transition from a liquid-like state to solid-like and vice versa (41, 42).

The most recent effort was reported by A. Gnoli et al. (43). Following the numerical simulation results by Capozza et al. (44; 45), which suggested that viscosity is reduced when the applied vibrating frequency is in a special range, they experimentally investigated the fluidization of a granular material subject to mechanical vibrations. As the vibration frequency is slowly increased, they observed a reentrant transition. A jammed system will first be fluidized, and then will return to a frictional state. The first transition to the fluidized state at low driving frequency regime is independent of the material properties and can be characterized by the detachment condition, which is estimated by the theory introduced by Capozza et al. (44, 45). The viscosity recovery transition, however, depends on the material properties. They suggested that the dependence originates from the balance between the acting dissipation and the inertial force in the system. Their numerical simulation results also support the former claims. The series of works introduced in this paragraph opens the possibility of controlling the viscosity in granular media.

\subsubsection{Phase separations in granular system}

In driving regimes, the fluidized granular system could also evolve to coexistent states from a homogeneous state. For example, in a vertically or horizontally vibrated 2D system with beads vertically packed, the topper layers could be fully fluidized, while the bottom parts still maintain crystalline structure (46, 47). In 2005, A. Götzendorfer and his coauthors observed sublimation, coexistence of gas and solid, in a monolayer system (48). The system was vertically vibrated and meanwhile rotated horizontally to demonstrate that the coexistence state was not introduced by the inhomogeneous distribution of forcing, or a tilt of the apparatus. Their MD simulations also confirmed their experiment. In the coexistence state, density of the solid part is several times of the gas part, while, the granular temperature is orders of magnitude lower. The most mysterious point is that, after enough relaxation time, a unique, time independent state with a constant solid fraction is established. However, why and how a constant fraction is established was still an open question. In 2008, M. C. Clerc et al. detailedly studied the transition process from liquid-like to solid-like in a 1D system (41). They found that the transition is mediated by waves and triggered by a negative compressibility, as for van der Waals phase coexistence. The phase separation is always concomitant with a pressure plateau, and similar plateaus also existed in their MD simulation results for $2 \mathrm{D}$ and $3 \mathrm{D}$ systems. Predominantly, a governing equation was derived in their work to describe the transition 
process. It exhibits similar dynamics to those shown by experiments and MD simulations. Moreover, the mapping of model parameters enable them to explore the phase diagram and observe richer phenomena. Later, Klaus Roeller first observed the liquid-gas phase separation in a quasi $2 \mathrm{D}$ shaking dry granular system by MD simulations. This phenomenon was confirmed by Jame Clewett in his experiments. Their results was presented in the work (49). They found that the emergence of a liquid-gas coexistence state out of a homogeneous initial condition is due to a process similar to spinodal decomposition in equilibrium fluids, in the region of the phase diagram where the effective compressibility becomes negative. The denser part is held by a surface tension resulting from the kinetic energy part of the pressure tensor (50).

\subsubsection{Granular system with extra interactions}

A work reported by D. J. Scheeres et al. (51) proposed that van der Waals should be the dominant force between the regolith grains on the surface of an asteroid. Asteroids are normally recognized to be composed of aggregates of smaller bodies with a surface being dominated by loose materials: grains and powders of different sizes. In the extreme environment of space, surfaces are much 'cleaner' than on Earth due to the lack of absorbed molecules on the surfaces of materials, allowing for closer effective distances between the regolith grain surfaces. In the case of extremely close contact, the strength of van der Waals forces can become quite strong and be greater than the electrostatic and solar radiation pressure forces and so on. Hence, it should be the van der Waals forces which prevent the materials on the surface from escape in regimes of extremely low ambient gravitational acceleration (51).

As a wetting liquid is added into the dry granular system, a liquid surface may extend from one particle to another and generate capillary bridges among particles. The capillary bridges will act on particles as an attractive force when they want to separate. This kind of bridge generates when two particles come into contact, and it can rupture at a certain critical distance $s_{c}$. Similarly to the dry case, the wet granular gas will cluster freely in case of no external driving $(52,53)$. The difference is that wet grains cluster by directly adhering to each other by capillary bridges as soon as the kinetic energy of involved grains are below the capillary bridge energy $E_{c b}$. Instead of condensing in momentum space, as is the case for dry granular gases, the wet granular gas condenses in real space (see Fig. 1.6p). When driving is introduced, a wet granular system may undergo a forcedriven solid-fluid transition and an energy-driven fluid gas transition, with coexistence states near the transition boundary lines (54, 55). With sufficient liquid fraction added, 
wet granular particles may construct a pile held together by the individual capillary bridges (56). In the case of larger liquid content, most capillary bridges merge, forming larger liquid clusters, and slurry. In many cases, the granular particles in slurries behave as the dry ones except for the higher viscosity (57, 58). However, when an appropriate secondary liquid is added, which creates capillary bridges among solid particles, the slurry may acquire a pasty consistency and turn into an elastic gel (59).

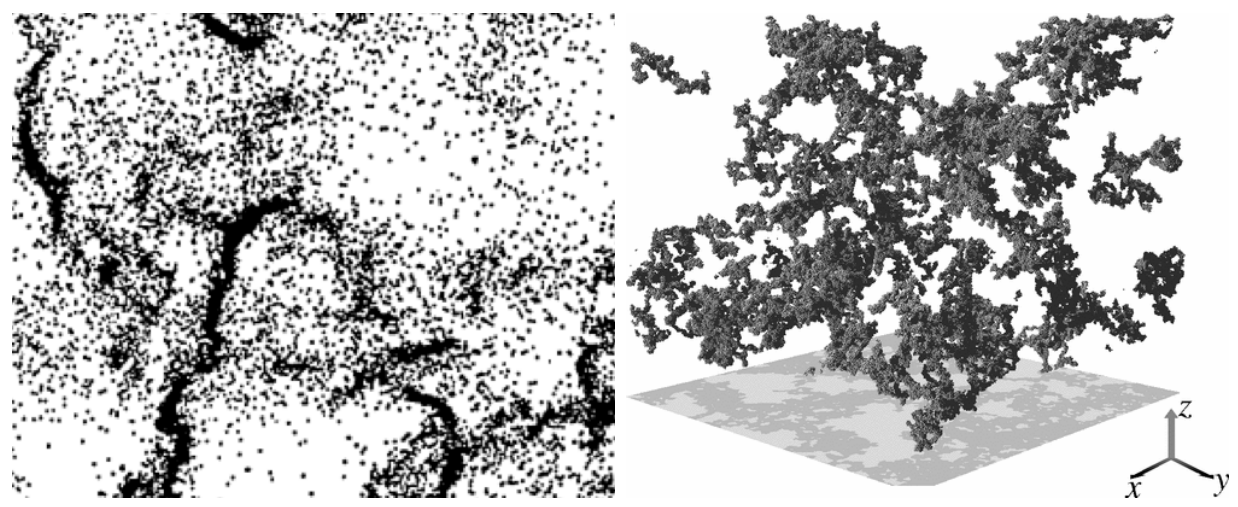

Figure 1.6: The clustering phenomena in dry (left) and wet (right) granular gas. Reprinted from (60; 52,53$)$.

\subsection{Research methods}

\subsubsection{Theoretical}

To develop a complete fundamental theory which could generally describe the phenomena observed in granular systems, considerable efforts have been focused on attempting to generalize the classical kinetic theory of gases to include dissipative collisions. Theories for the velocity distribution function and generalization of the Navier-Stokes equations for granular hydrodynamics have been well developed for low density, rapidly flowing granular gases, where pair collisions are dominant. A large number of authors contributed to this important theoretical advance. We refer the author to the book by $N$. V. Brilliantov and T. Pöschel (61) for more details. 


\subsubsection{Experimental}

Experimental setups used in the investigation of granular materials can vary greatly in different areas of experimental research. However, the usual means to detect the inner configurations of a granular system typically involve high-frequency cameras, used to take snapshots of the system which is visualized by X-ray or other lasers with appropriate frequency; an image processing is also needed to extract detailed information about contacts, volume fractions and so on. As it is enormously difficulty to visualize the internal state of a granular system, specialized materials or slow scanning times are highly needed.

Recently one new means, acoustic monitor, was proposed by T. A. Brzinski III (62) which can be used to perform 3D fast measurements without optical access to the system. The idea is based on the following. The density of vibrational modes $D(\omega)$ could describe important information of the state of the system. For example, the low-frequency modes develop upon approaching the jamming transition (63). With acoustic monitoring, they effectively measured the number of modes excited, instead of the the acoustic power among the modes with different frequencies. They found that the mode distribution broadens before and after a failure in granular system. Their results provide a possibility to forecast a failure like earthquakes or brittle fracture.

\subsubsection{Numerical}

Since there is not yet a comprehensive theory of granular materials, numerical simulations are one of the most reliable means to predict the behavior of such system. More importantly they can also be used to directly compare with the experimental results and help to improve the present theories.

One method based on a continuum approach and frequently used to study granular flows is called granular hydrodynamics (GHD). This method directly simulates the hydrodynamic equations and has a great power in predicting collective phenomena such as shocks, vortices and clusters appearing in granular flows. Another numerical method can be used to study granular system is the well known Direct Simulation Monte Carlo (DSMC). The main ideal of DSMC is to decouple the particle motions and collisions. The propagation of each particle is determined by its own position, velocity and the statistical distribution of the properties of the whole system, which can be achieved by solving the Boltzmann or Boltzmann-Enskog equation. Even though DSMC is more efficient than other meth- 
ods, it is still not frequently used in granular research as it is not always justified for non-homogeneous systems.

The most popular numerical method in numerical simulations of granular matter is the Discrete Element Method, also known as Molecular dynamics (MD). There are two ways to do MD simulations: one is called 'time-driven' MD, the other one is 'event-driven' MD. In the time-driven $\mathrm{MD}$, the exact trajectory of each grain is computed based on Newton's equations of motion. This method allows us to simulate both dynamic and static systems, and also systems with multi-particle contacts and complex-shaped particles, as long as the interaction forces between particles are known. As the governing equations need to be solved for each particle with small time intervals, this method is restricted to small system sizes. Luckily, modern technological advances such as graphics processing unit (GPU) make it possible to reach large systems. Compared to the time-driven MD, evendriven MD is more efficient as only the states before and after a collision are considered and the collisional details are ignored. This method, however, only applies to the system where the dynamics are mainly determined by pair collision. The time-driven MD is the method we implement in the work reported in this thesis; more details will be explained in the following chapters.

\subsection{Aims and scope of the thesis}

\subsubsection{Aims}

For granular particles with sizes ranging from 1 to $100 \mu \mathrm{m}$, the van der Waals force, is recognized as playing an important role. Different from the capillary bridge force which is hysteretic and dissipative, the van der Waal interaction is conservative adhesive force. The capillary bridges introduce both complexity, and significantly new properties into the granular system. The 3D space extended by van der Waals interactions, as sketched in Fig. $1.7(\underline{64})$, may have more abundant phenomena which are worth exploring.

Recently works involving van der Waals interactions, however, mainly focus on the freely cooling process of a system with attractive potentials( $(65)$, or on how a short-ranged attractive potential influences the dissipation in a granular system( $(\underline{66})$. The van der Waals interaction between macroscopic objects has been also considered in engineering settings, but mainly in the context of the packing of fine grains. Little to no effort yet 


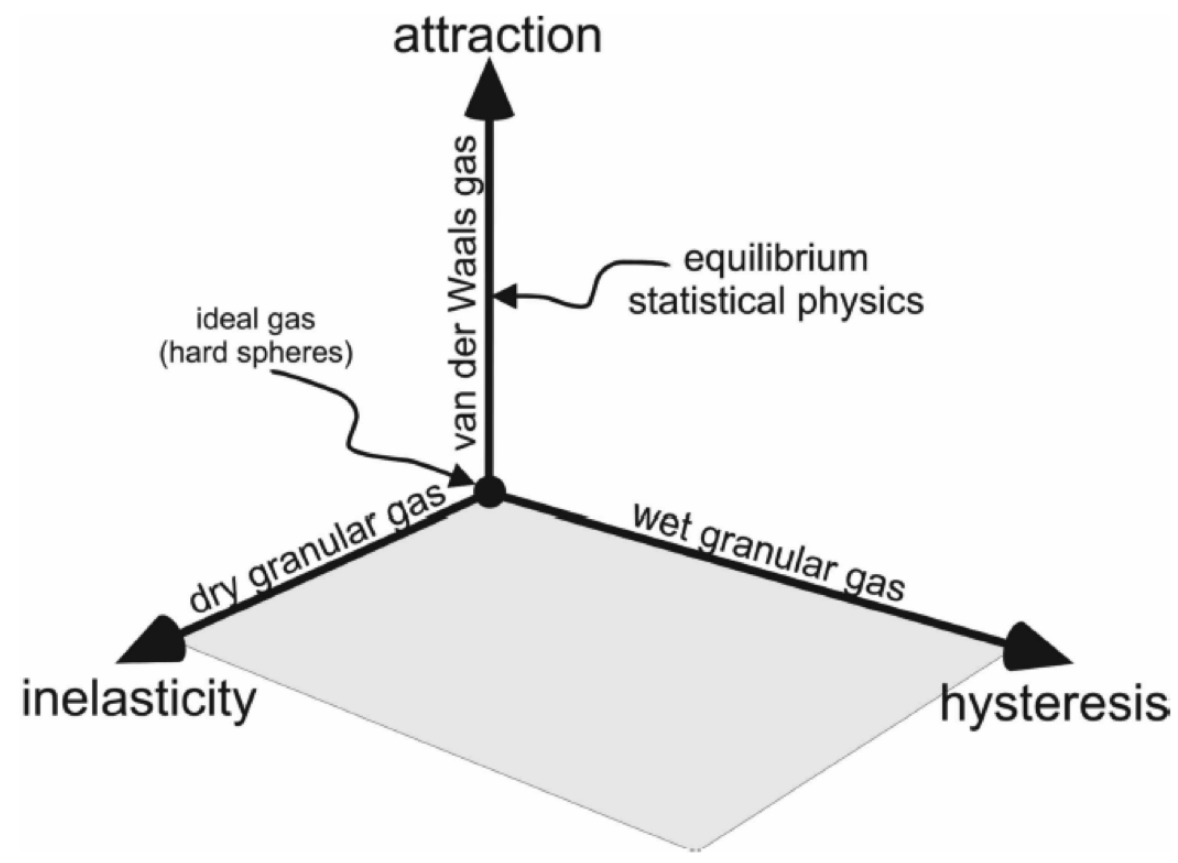

Figure 1.7: The sketch of the possible force space for granular system. Along the vertical axis, standard statistical mechanics is valid. However, it will break down when the inelasticity and hysteresis were introduced in. Reprinted from (혀). 
has been directed to study the influence of the van der Waals interaction on a granular gas system.

The work reported in this thesis tries to fill this gap by focusing on how van der Waals interactions influence the collective behavior of excited granular materials. In our system, a number of identical granular spheres are confined between two separated walls in a zero gravity environment, and the whole system is vibrated in the direction perpendicular to walls. We numerically study the system with MD and the van der Waals interactions are included based on the real size of the grains. We find a new interesting solid and gas coexistence state induced by the van der Waals interactions; the solid component in the coexisting state can be either random close packing or poly-crystalline (with fcc and hcp local structures).

\subsubsection{Scope}

The thesis is organized as follows. Following this introduction about the physical background, Chapter 2 explains the details of the MD simulations we used in the reported work and the quantities we selected to characterize the states. The detailed information of the simulated system is also shown in this part. In Chapter 3 we show the phase diagrams for a driven granular system without van der Waals interactions, the prediction of the regime where van der Waals interaction may play a significant role, and some simulation tricks to clearly determine the phase boundaries in an cohesive granular system. The phase diagram for a driven system with van der Waals interactions are shown in Chapter 4 together with snapshots, and the characteristics of all possible phases in the explored phase space. Chapter 5 explains the evolution processes of different phases and discussed the detail structure and the stability of crystallized component in the coexistence state. In Chapter 6 we focus on the two important physical factors: restitution coefficient and the Hamaker constant, and show how they influence the boundary line between the two different coexistence states. The last Chapter collects our conclusions and provides a brief outlook for possible future work. 



\section{Theoretical and computational methods}

The collective behavior of granular materials stems from the interactions among individual particles and the interactions with the surrounding environment. Therefore, a particlescale approach based on the detailed mechanism of particle interactions is crucial for a quantitative understanding. Several computational techniques have been developed and are widely used to support such research, including the direct numerical simulations of the hydrodynamic equations, Monte Carlo methods, and the molecular dynamics(MD) simulations.

There are two ways to do MD simulations, one is the time-driven MD, i.e., using a soft model, the other is the event driven MD, i.e, using a hard sphere model. The soft-sphere or time-driven MD method was developed by Cundall and Strack in 1979 (67). They assumed that particles are 'soft' and can experience deformations. Particles repel each other in response to the deformation and the stronger the deformation is, the harder they will repel each other. So, the deformation can be used to describe interactions between particles, such as elastic, inelastic or frictional forces. In this approach, multiple-particle interactions can be easily handled and the method is not limited to perfect spherical particles. For dilute or rapidly flowing systems, 'hard core' or event-driven MD models are instead widely used (68). The interaction is assumed instantaneous and only one collision at a time is considered. Because the forces affect the dynamics only upon mechanical contact, the forces between particles do not need to be explicitly considered, and only the outcome of the collision matters. Therefore, in this method, a sequence of collision 'events' is sequentially processed.

The system we studied in this thesis is comprised of granular particles with cohesive van der Waals interactions, so it is prone to become dense and multi-particle interactions may became dominant. Hence we choose to employ time-driven MD. In the remainder of this Chapter, we will explain the details of time-driven MD method, including the 
governing equations we used, the forces models, and also some computational details. Afterwards, we will introduce the geometrical and physical parameters of our system and the observables used to characterize the phases.

\subsection{Governing equations}

A granular particle can have two types of motion: translational and rotational. During its movement, it may collide with other particles, walls, be influenced by gravity, possibly a surrounding fluid, and other potential fields such as electromagnetic field, van der Waals interactions, and so on. To describe the motion of each individual particle, Newton's second law is needed. For a particle $i$ with mass $m_{i}$ and moment of inertia $I_{i}$, the governing equation can be written as

$$
\begin{aligned}
m_{i} \frac{d \mathbf{v}_{i}}{d t} & =\sum_{j} \mathbf{F}_{i j}^{c}+\sum_{k} \mathbf{F}_{i k}^{n c}+\mathbf{F}_{i}^{w}+\mathbf{F}_{i}^{f}+\mathbf{F}_{i}^{g} \\
I_{i} \frac{d \boldsymbol{\omega}_{i}}{d t} & =\sum_{j} \mathbf{M}_{i j}
\end{aligned}
$$

where $\mathbf{v}_{i}$ and $\boldsymbol{\omega}_{i}$ are respectively the translational and angular velocities of particle $i, \mathbf{M}_{i j}$ is the torque acting on particle $i, \mathbf{F}_{i j}^{c}$ the contact force on particle $i$ due to particle $j$, $\mathbf{F}_{i k}^{n c}$ is the non-contact force acting on particle $i$ due to particle $k, \mathbf{F}_{i}^{w}, \mathbf{F}_{i}^{f}$ and $\mathbf{F}_{i}^{g}$ are the force induced by the wall, by the surrounding fluid and gravity, respectively. In Fig. 2.1. we give a sketch of possible forces acting on one particle.

In the MD method, during a small time interval $\delta t$, a perturbation by one particle cannot reach the region outside its range of interaction. We call $\delta t$ simulation time step. Once all the forces in Eqs. (2.1) are known, the governing equations are numerically solvable within one time step. Thus, the trajectories, velocities and all the forces acting on the particles in the system can be determined.

The reported work in this thesis is about a granular system with van der Waals interaction confined by walls in the zero gravity environment, so the governing equation can be simplified to

$$
m_{i} \frac{d \mathbf{v}_{i}}{d t}=\sum_{j} \mathbf{F}_{i j}^{c}+\sum_{k} \mathbf{F}_{i k}^{n c}+\mathbf{F}_{i}^{w}
$$




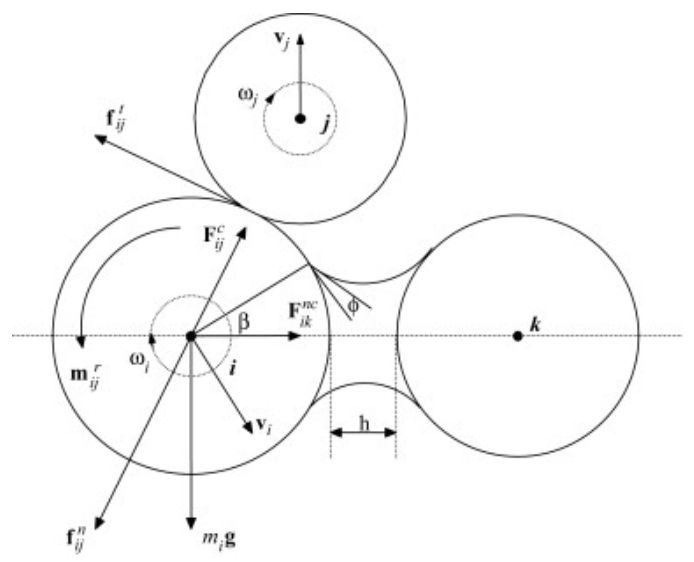

Figure 2.1: Diagrammatic sketch of forces acting on a granular particle $i$. Reprinted from (69)

\subsection{The force models}

\subsubsection{Particle-particle contact force}

The 'soft' sphere method assumes that two particles will slightly overlap with each other during a collision. This is reasonable as the contact between two particles is not just in one single point but on a finite surface due to the deformation. It is difficult to trace the interaction details over the surface accurately and generally, as it is related to many geometrical and physical factors such as the shape, material and kinematic state of particles. Hence, to be computationally efficient and applicable in multi-particle systems, simplified force models are generally used to describe the particle contacts for time-driven MD simulations.

Considerable effort has been devoted to describe granular contact forces between spherical particles. The most intuitive and simple one is the linear-dashpot model introduced by Cundall and Strack in 1979 (67), where the spring accounts for the elastic deformations and the dashpot for the viscous dissipation. A more complex model, Hertz-Mindlin and Deresiewicz model has also been developed. The elastic contact between two spheres in the normal direction was introduced by Hertz in 1882 (70). He considered that the the dependence of normal force on the normal displacement is nonlinear. Mindlin and Deresiewicz included also a tangential force in 1953 (71). They demonstrated that the relationship between the force and displacement is history-dependent and depends on the instantaneous rate of force and displacement variation. The details of this model were 
reviewed by Vu-Quic and Zhang (72) in 1999 and Di Renzo and Di Maio (73) in 2004. Even though it is more practical, this model is not widely adopted in MD simulations as it is too complicated and time-consuming for a granular system involving a large number of particles.

Various simplified models based on The Hertz-Mindlin and Deresiewicz theories have been developed. Walton and Braun (74) used a semi-latched spring force-displacement model in the normal direction. In the tangential direction, they used the case constant normal force in the Mindlin and Deresiewicz theory (71). Thornton and Yin (75) adopted the Hertz theory for the normal direction, but a more complex model for the tangential part. They assume the tangential displacement depends on the variation of the normal force. Langston et al. (76) used a more intuitive model, in which the the Hertz theory is used for the normal force and direct force-displacement relation is used for the tangential force. The equations of the above force models for spherical particles can be found in the review work by H.P. Zhu et al. (69).

The tangential force or asymmetrical normal forces on a particle will generate a torque which induces rotations. The torque caused by the normal component, usually called rolling friction, is still difficult to determine. The torque plays a significant role during the transitions from static to dynamical states, such as the formation of shear band, heaping and a moving particle on a plane. In most other cases, the frictional torque is negligible.

The contact forces between non-spherical particles are far more complex. For the particle with irregular shape, a feasible method is to assume that the particle's surface is a collection of small node-particles. With this method, very complicated shapes can be handled, and only one contact model for spherical particles is required. For a given well-known regular shape, such as ellipsoid, cylinder or tetrahedron, solvable mathematical equations can be used to determine if there is a contact or not between one particle and its neighboring particles. Then the contact model for spherical particles can be used to calculated the contact force. This approach is more accurate but still computationally expensive.

Theoretically, the non-linear models based on the Hertz and Mindlin-Deresiewicz theory should be more accurate than the simple linear model. However, the investigation by $D i$ Renzo and Di Maio (73) showed that the simple linear model gives even better results than the nonlinear ones in some cases. That's probably because the theory is often based on the geometrically ideal particle, which is not practical. Besides, the accuracy of the model highly depends on the selection of parameters. 
The work reported in this thesis deals with a system composed of perfectly spherical granular particles with diameter $d$. The system evolves from a dilute homogeneous gas, so the effects of torques are neglected and only the head-on collisions are considered. With considerations of computational efficiency in mind, the linear dashpot is chosen to model the contact force. The collision force between particle $i$ and $j$ at positions $\mathbf{r}_{i}$ and $\mathbf{r}_{j}$ is then written as follows

$$
\mathbf{f}_{i j}^{c}=\left[-k\left(d-\left|\mathbf{r}_{i j}\right|\right)+\mu \mathbf{v}_{i j} \cdot \hat{\mathbf{r}}_{i j}\right] \hat{\mathbf{r}}_{i j},
$$

where $k$ is the spring constant, $\mu$ the damping coefficient, $\mathbf{r}_{i j} \equiv \mathbf{r}_{j}-\mathbf{r}_{i}, \hat{\mathbf{r}}_{i j} \equiv \mathbf{r}_{i j} /\left|\mathbf{r}_{i j}\right|$, and $\mathbf{v}_{i j} \equiv \mathbf{v}_{j}-\mathbf{v}_{i}$.

\subsubsection{Particle-particle non-contact forces}

In reality, the interactions between granular particles are not just dissipative collisions. For grains of different sizes or physical properties, additional non-contact interactions appear and can became dominant in certain conditions and change the collective behavior of granular materials. The capillary bridges between particles in a wet granular system make it possible to build up a sand sculpture, while grains can flow like a liquid when the liquid component increases past a threshold. Importantly, electrostatic interactions in flows composed of charged grains can enhance or suppress clustering effects. Some empirical quantities such as the Hausner ratio (a measure of flowing ability for powders), angle of repose, shear stress can be used to explain some behaviors of granular particles. But general quantitative application is difficult. As a method which could includes the information down to each particle, these non-contact forces can be also directly considered by DEM.

In the work by Rumpf (77) in 1962, he compared how the relative importance of typical non-contact forces varies with particle's size, as illustrated in Fig. 2.2. Van der Waals force and capillary force decrease with decreasing particle size. Nevertheless, the strengths of gravity and electrostatics decrease even faster. Consequently, for the system with granular size ranging from 1 to $100 \mu \mathrm{m}$, van der Waals interactions and capillary force become dominant. That is the reason why powders (even the dry one) are said to be cohesive and not easily fluidized. A lot of work has been done for wet granular system. Here we only consider the van der Waals interactions in dry granular system, and the only non-contact force is the van der Waals interaction. 


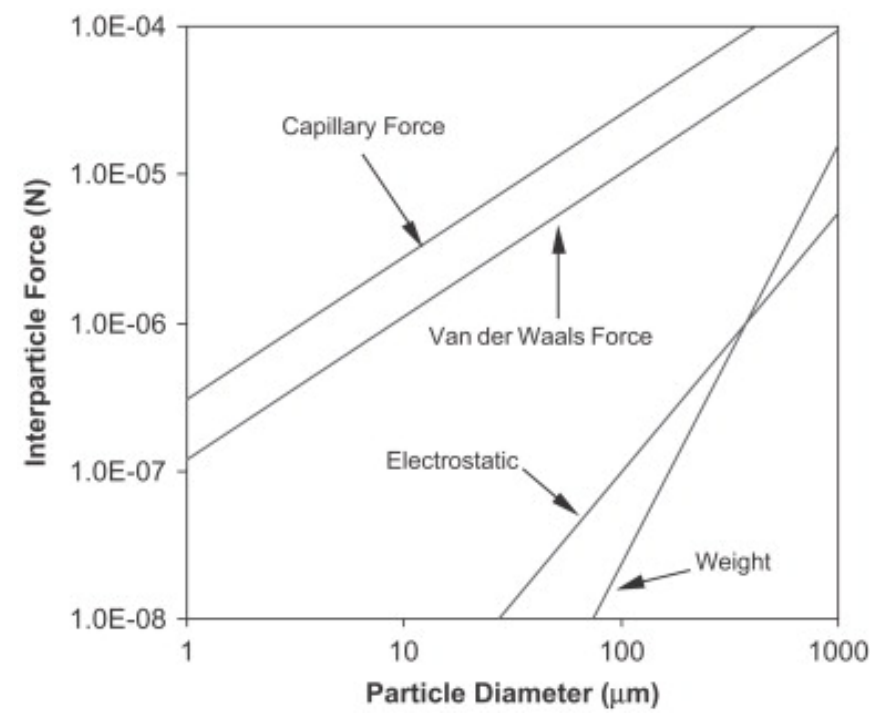

Figure 2.2: Comparison between several non-contact forces. Reprinted from (77).

The van der Waals force is also know as "charge-fluctuation" force or "electrodynamic" force. It turns out that such forces are for more powerful within and between condensed phases - liquids and solids - than they are in gases. In fact, they frequently create condensed phases out of gases. In a material body or in a vacuum, transient electric and magnetic fields always arise spontaneously, as in all matter positive and negative charges are constantly fluctuating. These fluctuations in charge and in field occur not only because of thermal agitation but also because of quantum-mechanical uncertainties in the positions and momenta of particles and in the strengths of electromagnetic fields. The momentary positions and electric currents of moving charges act on, and react to, other charges and their fields. It is the collective coordinated interactions of moving electric charges and currents, and fields, averaged over time, that create the van der Waals interaction (78).

For both practical and fundamental reasons, there was the need to learn about the interactions of bodies much larger than atoms and molecules. In 1937, H. C. Hamaker (179) investigated the properties of van der Waals interactions between large bodies, based on previous work dealing with molecular interactions. His theory, now known as the Hamaker theory, is based on the assumption of "pairwise additivity". The idea of this approximation is that incremental parts of large bodies could interact via energies scaling as $-1 / r^{6}$ as though the remaining material were absent. According to this theory, the interaction energy of two spheres, approaches $1 / r$ power near contact, and reverts to the 
power $1 / r^{6}$ when the separation is much wider compared with their size. The coefficient of the interaction came to be known as the Hamaker constant $H$.

A shortcoming of Hamaker's theory is that it neglects many-body interactions and retardation effects. In 1950s, Lifshitz (80) considered the van der Waals interaction acting between macroscopic bodies rather than the summed interaction of atoms and molecules. It used the relation between absorption spectra and fluctuations, considered the real material between bodies and the retardation mechanism. This theory is rigorous only at the separations larger than the atom or molecules of the interacting bodies. As each body is treated as a continuum in this approach, the rigor comes at a price.

To simplify our calculations, we choose the Hamaker theory which is commonly used in MD simulations to model the van der Waals interaction. The van der Waals force between two macroscopic spheres $i$ and $j$ with the same diameter $d$ can be calculated as

$$
\mathbf{f}_{i j}^{n c}=\mathbf{f}_{i j}^{v d w}=-\frac{H d}{24 h_{i j}^{2}} \hat{\mathbf{r}}_{i j}, \quad \text { for } h_{i j}<\frac{d}{4},
$$

where $h_{i j}$ is the surface-to-surface separation between particles $i$ and $j$ in the normal direction. Because the force drops off rapidly with increasing separation, we ignore the van der Waals force when $h_{i j}>d / 4$. The Hamaker constant $H$ is geometry and material dependent. It is usually given in units of zeptojoules (zJ): $1 \mathrm{zJ}=10^{-21} \mathrm{~J}=10^{-14} \mathrm{erg}$. This coefficient, calculated in natural units, typically ranges from 1 to 100 times the thermal energy $k_{\mathrm{B}} T_{\text {room }}=1.3807 \times 10^{-23}(\mathrm{~J} / \mathrm{K}) \times 293 \mathrm{~K} \approx 4.05 \mathrm{zJ}$.

The divergence of the van der Waals force in Eq. (2.4) for vanishing distances derives from the macroscopic treatment of dispersion forces under which it was derived (79); to remove this divergence, the force is cut off at $h_{0}=1 \mathrm{~nm}(\mathbf{8 1}, 82,83)$; then for distances $h \leq h_{0}, \mathbf{f}_{i j}^{n c}(h)=\mathbf{f}_{i j}^{n c}\left(h_{0}\right)$.

\subsubsection{The particle-wall forces}

The system we study here is a dry granular gas, with no interstitial fluid and no gravity. The only interaction with the environment is through the collisions with confining solid walls. Energy is injected into the system to maintain a steady state via the collisions between particles and walls.

Here we assume the walls are infinitely deep and hard with infinite mass, so that the particles cannot penetrate them and only the particles are deformed during the collisions. 
Friction with the walls can be neglected when the walls shake rapidly. We again use the spring-dashpot model to describe the collisional force between a particle $i$ and one of the walls $w$.

$$
\mathbf{f}_{i}^{w}=k \delta\left[\frac{d}{2}-\left|z_{w}-z_{i}\right|\right] \hat{\mathbf{z}}+\mu\left(\mathbf{v}_{w}-\mathbf{v}_{i}\right) \cdot \hat{\mathbf{z}},
$$

where $z_{i}$ and $\mathbf{v}_{i}$ are the $z$ coordinate and velocity of particle $i$ upon collision with the wall, $z_{w}$ and $\mathbf{v}_{w}$ are the $z$ coordinate and velocity of the wall, and $\delta=-1(+1)$ for the top (bottom) wall.

According to the Hamaker theory, the van der Waals interaction between particle and wall can be modeled as

$$
\mathbf{f}_{p w}^{v d w}=-\frac{H d}{12 l}, \quad \text { for } l<\frac{d}{2}
$$

where $l$ is the particle-wall surface separation.

As we know, the van der Waals field is conservative. Two cases may happen when a particle with kinetic energy $E_{k i n}$ enters a van der Waals field with potential energy $U$. It may be trapped and cannot escape from the field when $E_{k i n}<U$, or it will just go through the field and come out with the same $E_{k i n}$. The maximum kinetic energy $E_{k i n}^{c}$ one particle can gain after a collision with a shaking wall (whose position changes with time according to $A \sin (\omega t))$ is $\frac{1}{2} m(A \omega)^{2}$. For a realistic choice of parameters, this is much larger than the potential energy obtained from the Eq. 2.6, , hence we can neglect the van der Waals interaction between particle and wall.

\subsection{Structure of the simulation algorithm}

In Fig. 2.3, we give a schematic structure of the MD program used to simulate a driven granular system with van der Waals interactions. The simulation starts from time $t_{0}$ and ends at $t_{\max }$, with a time step $\delta t . t_{s}$ is a quarter of the driving cycle. $r$ and $v$ are the position and velocity of any given particle in the system. The neighboring particles which may act on the selected particle are determined through a linked list. All the states of particles are propagated to the next time step according to the position Verlet algorithm. As shown in the sketch map, we output one snapshot each $t_{s}$ for the calculation of statistical averages. 


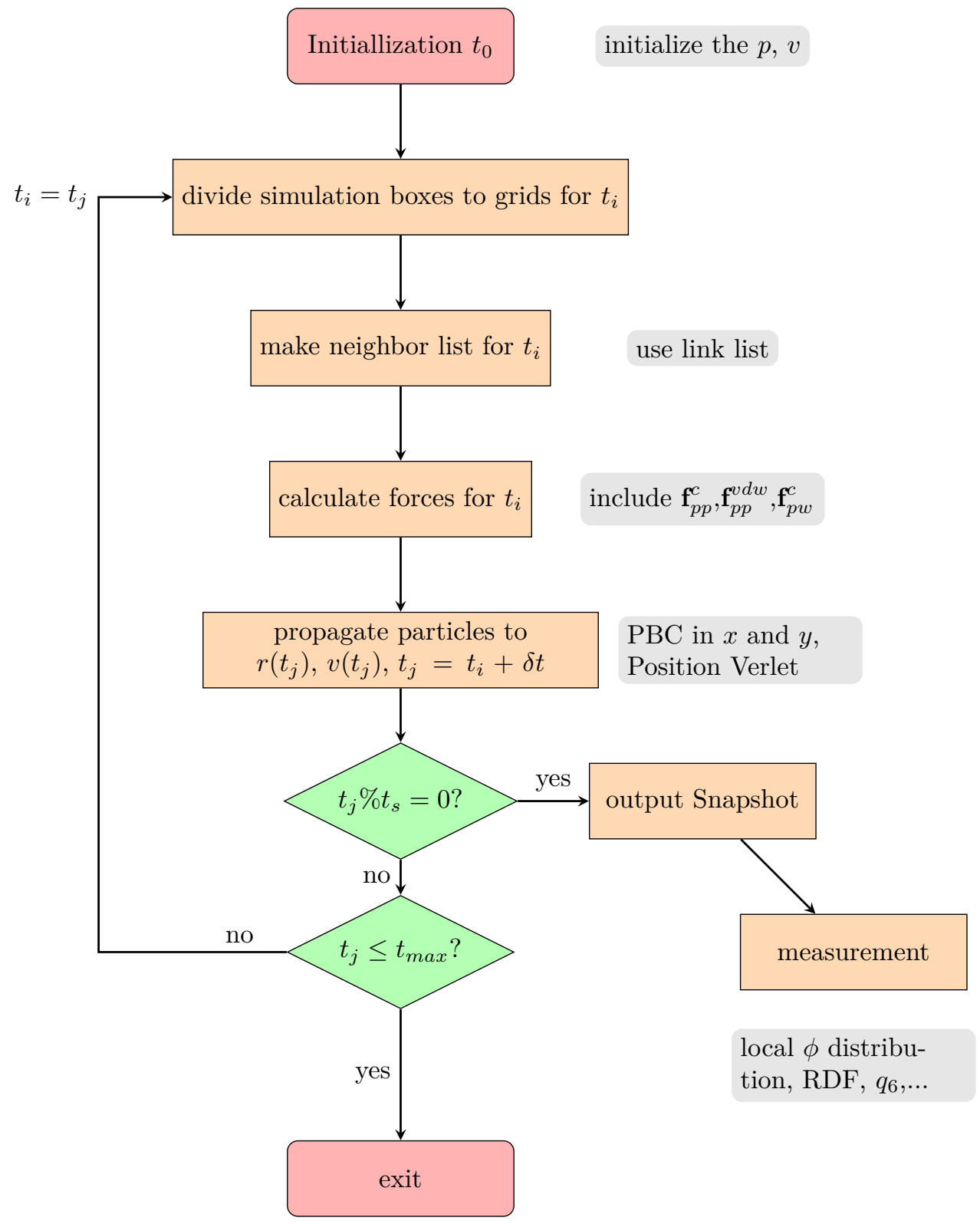

Figure 2.3: The schematic structure of our MD program to simulate a driven granular system with van der Waals interactions. 


$$
n-1
$$

$n+1$

$n$

$n+1 / 2$

$n+1$

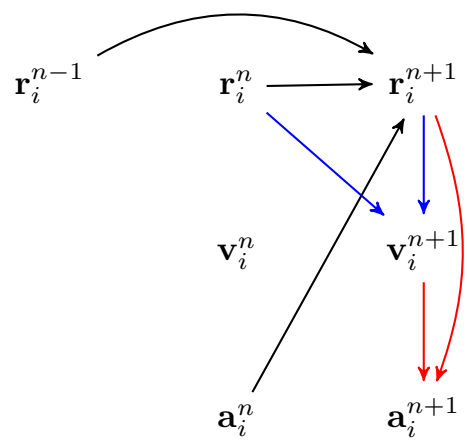

(a) Position Verlet.

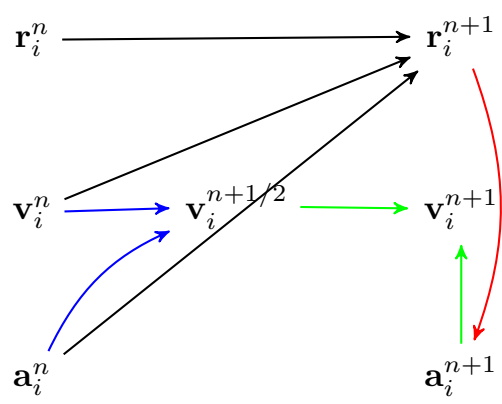

(b) Velocity Verlet.

Figure 2.4: Position and velocity forms of the Verlet algorithm.

\subsubsection{The particle propagation}

For one particle $i$, with position $\mathbf{r}_{i}$, velocity $\mathbf{v}_{i}$ and acceleration $\mathbf{a}_{i}$, the basic numerical scheme to propagate the particle's position from step $n$ to next time step $n+1$ is the Euler method.

$$
\begin{aligned}
& \mathbf{r}_{i}^{n+1}=\mathbf{r}_{i}^{n}+\mathbf{v}_{i}^{n} \delta t, \\
& \mathbf{v}_{i}^{n+1}=\mathbf{v}_{i}^{n}+\mathbf{a}_{i}^{n} \delta t
\end{aligned}
$$

The Euler method (84) is a first-order approximation method, which means that the local error per step is proportional to the square of the step size. It is known to be numerically unstable.

Two well-known, more stable algorithms were developed by Verlet (85, 86), and are schematically shown in Fig. 2.4. The first one is the position-Verlet algorithm (shown in Fig. 2.4a; this updates the positions of particles according to the rule

$$
\mathbf{r}_{i}^{n+1}=2 \mathbf{r}_{i}^{n}-\mathbf{r}_{i}^{n-1}+\mathbf{a}_{i}^{n} \delta t^{2}
$$

that is, the new position $\mathbf{r}_{i}^{n+1}$ only depends on the acceleration $\mathbf{a}_{i}^{n}$ and the two previous positions $\mathbf{r}_{i}^{n}$ and $\mathbf{r}_{i}^{n-1}$. The new velocity can be calculated from

$$
\mathbf{v}_{i}^{n+1}=\frac{\mathbf{r}_{i}^{n+1}-\mathbf{r}_{i}^{n}}{\delta t}
$$


The second scheme is the velocity-Verlet (shown in Fig. 2.4b). The standard implementation scheme of this algorithm is as follows. The new position is calculated from

$$
\mathbf{r}_{i}^{n+1}=\mathbf{r}_{i}^{n}+\mathbf{v}_{i}^{n} \delta t+\frac{1}{2} \mathbf{a}_{i}^{n} \delta t^{2}
$$

Next, the new acceleration $\mathbf{a}_{i}^{n+1}$ is calculated from the interaction potential using the updated positions $\mathbf{r}_{i}^{n+1}$. Then the new velocity is derived from

$$
\mathbf{v}_{i}^{n+1}=\mathbf{v}_{i}^{n}+\frac{\mathbf{a}_{i}^{n}+\mathbf{a}_{i}^{n+1}}{2} \delta t
$$

Note that this algorithm assumes that the acceleration $\mathbf{a}_{i}^{n+1}$ only depends on position $\mathbf{r}_{i}^{n+1}$, and does not depend on the velocity $\mathbf{v}_{i}^{n+1}$.

In our simulations of granular systems with the soft-sphere model, the collision forces between particles always depend on the particle's velocity. So for our simulation, the position-Verlet algorithm is chosen to propagate particles.

\subsubsection{The neighbor list}

In MD simulations, generally we need to consider a particle $i$ and search over all other particle $j$ to calculate the separations and determine whether they are within the range of interaction. If two particles are separated by a distance larger than the interaction range cutoff, the program will skip to next pair. The number of operations required to examine all pair separations scales as $N^{2}$. This can become prohibitively costly for large systems. In 1967, Verlet (85) proposed a technique to speed up the computation by building a list of neighbors for each particle, which is updated at intervals. During this time interval, only the separation between particles within the same group in the neighbor list are checked. The number of distances that have to be considered is thus significantly reduced.

\section{The Verlet neighbor list}

In the Verlet method, given the range of the interaction $r_{c}$, a cutoff $r_{l} \geq r_{c}$, is set before the simulation starts, see Fig. 2.5 For a given particle $i$, all the particles within a sphere of radius $r_{l}$ around $i$ are included into the neighbor list of $i$. The neighbor lists for all particles will be stored in a large array, named as LIST. A second array POINT of size $N$ (which is the total particle number in the system) is constructed at the same time. 


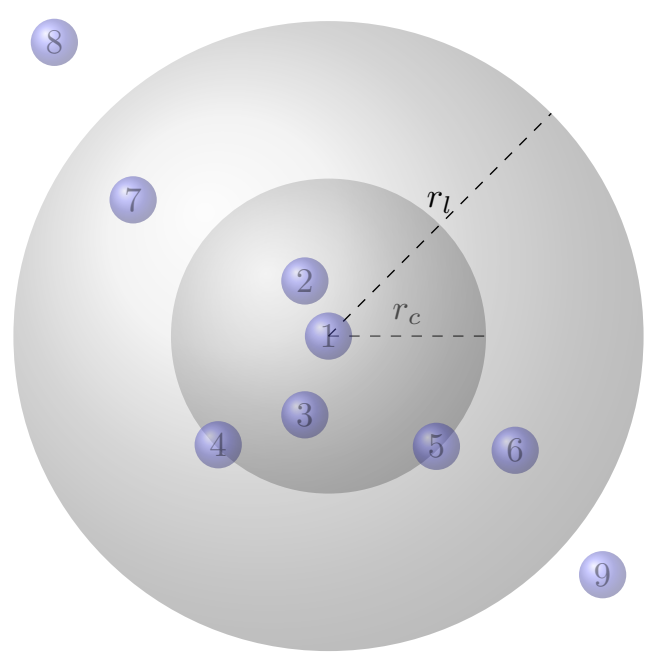

Figure 2.5: Diagrammatic sketch of neighbor list construction for particle $i . r_{c}$ is the interaction potential range. $r_{l}$ is the cutoff for the neighbor search. At the time when the list is constructed, particle 2, 3 are within the interaction range. Particle 4,5 and 6,7 are either outside the interaction sphere and within the cutoff sphere, or at the boundary line. They still have a chance to enter the interaction range, so they must be included in the neighbor list. Particle 8,9 are outside the cutoff sphere, they can't enter into the interaction sphere within the time interval before the list is updated. 
POINT [i] points to the first neighbor of particle $i$. All the neighbors of $i$ are stored in the entries from LIST[POINT[i]] to LIST[POINT [i] - 1].

The neighbor list is updated from time to time, usually after $10-20$ time steps. The cutoff $r_{l}$ is selected to make sure that, during the update interval no particle outside the neighbor list will crash into the inner sphere with radius $r_{c}$. The neighbor list can also be updated automatically. A vector $\mathbf{S}$ can be used to record the total displacement of each particle since last update. When the sum of the two largest number in $\mathbf{S}$ exceed $r_{l}-r_{c}$, the neighbor list should be updated again.

\section{The linked list}

For large system, another more efficient way to build a neighbor list is the cell index method combined with a linked list $(87,88)$. The whole simulation domain is divided into small cells with a uniform grid. One $3 \mathrm{D}$ simulation domain of size $L_{x}, L_{y}, L_{z}$ will be divided into cells of size $\frac{L_{x}}{B_{x}}, \frac{L_{y}}{B_{y}}, \frac{L_{z}}{B_{z}}$ where the size of the cell must be greater than the interaction range of individual particle. $B_{x}, B_{y}, B_{z}$ are the numbers of grid cells in the $x, y$, and $z$ directions, so that each cell has an index number between 0 and $N_{b}-1$, where $N_{b}=B_{x} B_{y} B_{z}$ in the total number of cells in the grid. A $2 \mathrm{D}$ example is shown in Fig. 2.6a.

First, all particles in the system have to be sorted into their proper cells. Then two arrays, called head with dimension $N_{b}$ and list with dimension $N$, are defined and initialized. We loop through all particles and meet the first particle $i$ in box $b$, then the head [b] will be set as $i$ and list [i] will be set as 0xff. When the second particle $j$ comes, head [b] will be set to $j$ and list [j] will be set to $i$ (see Fig. 2.6c and Fig. 2.6d). After the loop, each cell $b$ has its corresponding head particle head [b] and all other particles in the cell can be found one by one through the array list starting from list [head[b]] (see Fig. 2.6b. head [b]=0xff means that the cell is empty and list [i]=0xff means that all particles in the cell have been found.

In this method, the neighbor list of any given particle consists of all particles in the same cell and all its nearest neighbor cells (26 cells in $3 D$ case). The linked list algorithm is so fast at sorting all the particles into cells and building their neighbor lists, that it may be executed every time step. The system studied in this work includes from $1 \times 10^{4}$ to $2 \times 10^{4}$ particles, so the cell method combined with the linked list is used to construct the neighbor list. 


\begin{tabular}{|c|c|c|c|c|c|}
\hline 30 & 31 & 32 & 33 & 34 & 35 \\
\hline 24 & 25 & 26 & 27 & 28 & 29 \\
\hline 18 & 19 & 20 & 21 & 22 & 23 \\
\hline 12 & 13 & 14 & 15 & 16 & 17 \\
\hline 6 & 7 & 8 & 9 & 10 & 11 \\
\hline 0 & 1 & 2 & 3 & 4 & 5 \\
\hline
\end{tabular}

(a)

$$
\operatorname{head}[\mathrm{b}]
$$

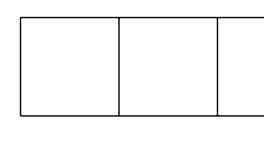

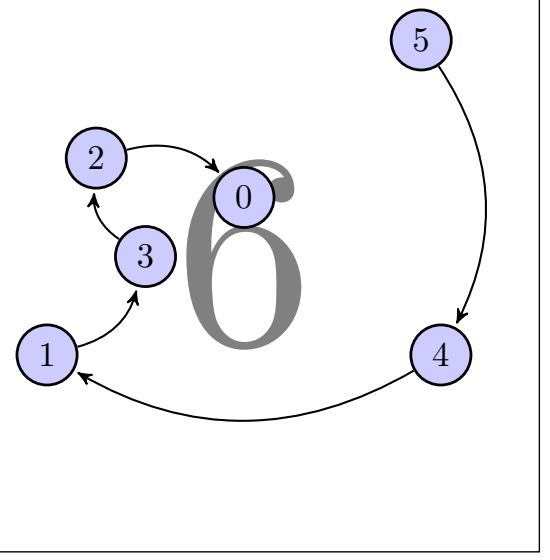

(b)

$$
\begin{array}{ccccccc|c|c|c|}
\text { box index } b & 0 & 1 & 2 & 3 & 4 & 5 & 6 & \cdots & N_{\text {box }} \\
\text { (c) } \\
\text { list[i] } & \text { Oxff } & 3 & 0 & 2 & 1 & 7 & 5 & &
\end{array}
$$

\begin{tabular}{|c|c|c|c|c|c|c|c|c|}
\hline $\operatorname{0xff}$ & 3 & 0 & 2 & 1 & 7 & 5 & & \\
0 & 1 & 2 & 3 & 4 & 5 & 6 & $\ldots$ & $N$
\end{tabular}

(d)

Figure 2.6: The diagrammatic sketch of the cell index method and linked list in 2D. (a) The simulation grids and their serial numbers. (b) The linked list inside the 6th grid cell where particle 5 is the head. (c)-(d) Part of the head and list arrays corresponding to (b). 


\subsection{Choice of parameters}

The system we study in this thesis is a confined granular system initially in a gas-like state. The granular particles have diameter $d=70 \mu \mathrm{m}$, density $\rho=8.9 \mathrm{~g} / \mathrm{cm}^{3}$ (the density of bronze) and are confined in the $z$ direction by two flat, parallel walls separated by a distance $L=18.5 \mathrm{~d}$. The whole system is vibrated sinusoidally with amplitude $A$ and frequency $\nu$ along the $z$ direction. The simulated domain has dimensions $L_{x}=$ $160.0 d, L_{y}=20.0 d, L_{z}=L$, and periodic boundary condition are used along the $x, y$ directions. The filling fraction $\phi$ is used to describe the averaged filling condition inside the simulated domain. It is defined as $\phi=\frac{N v_{p}}{L_{x} L_{y} L_{z}}$, where $N$ is the total particle number and $v_{p}$ is the volume of an individual particle.

The energy dissipation during particle pair-collisions is described by the restitution coefficient $\epsilon$, which is the ratio of the final to initial relative velocity between two particles. For the head-on collision considered in this thesis $\epsilon$ ranges from 0 to 1 , where 1 would be a perfectly elastic collision.

In the spring-dashpot model, each particle is treated as a damped harmonic oscillator. The governing equation for the deformation is

$$
m \ddot{x}=-k x-\mu \dot{x}
$$

where, $x$ is the deformation, $k$ is the Hooke's constant and $\mu$ describes the damping. The solution for Eq. 2.13) is

$$
x=e^{-\gamma t} \sin (\omega t)
$$

where $\gamma=\frac{\mu}{2 m}$, and $\omega=\gamma^{2}-\frac{k}{m}$. During a collision which starts at $t_{s}$ and finishes at $t_{e}$, the restitution coefficient is

$$
\epsilon=\left|\frac{\dot{x}\left(t_{s}\right)}{\dot{x}\left(t_{e}\right)}\right|
$$

with

$$
t_{s}=0, \quad t_{e}=\frac{\pi}{\omega}, \quad x\left(t_{s}\right)=x\left(t_{e}\right)=0 .
$$

Then, we can find the follow relation between $\epsilon$ and the damping parameter,

$$
\epsilon=e^{\gamma t_{e}}=e^{\gamma \pi / \omega}
$$

Equation 2.17 means that once we assign the experimentally accessible $\epsilon$ and $k$, we can find the corresponding $\mu$. 


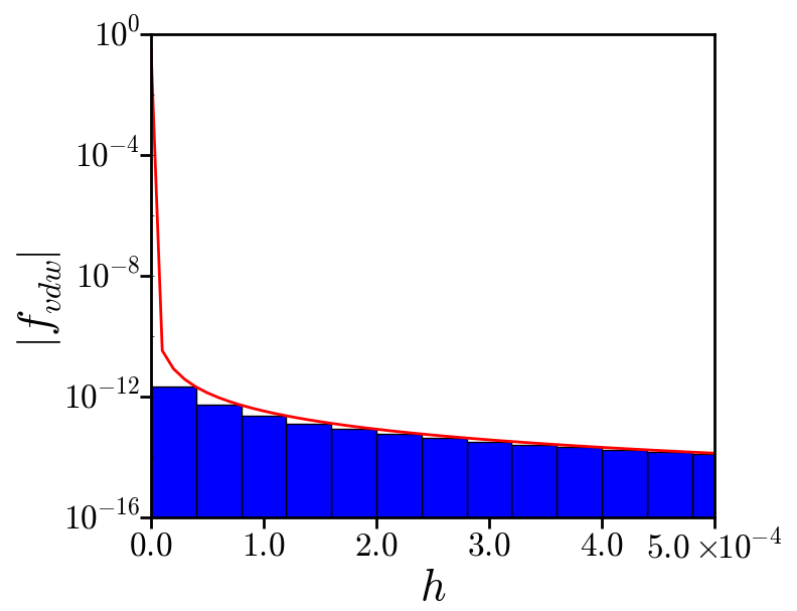

Figure 2.7: The two-particle van der Waals force from simulation (the blue histogram) and theory (red solid line). The blue shaded area is the absolute value of potential energy captured by the simulation.

The time step $\delta t$ we use is $10^{-6} \mathrm{~s}$, and all simulations presented here are run for $10^{8} \delta t$. The driving frequency $f=50 \mathrm{~Hz}$. The restitution coefficient $\epsilon$ is set to 0.9 and the Hamaker constant $H=8.0 \times 10^{-20} \mathrm{~J}$ if not otherwise mentioned. The spring constant is $k=0.5$ $\mathrm{N} / \mathrm{m}$ for both particle-particle and particle-wall collisions. With this choice of model parameters particles may experience a maximum overlap of $10 \%$ their radius.

\subsection{Van der Waals force shift}

As the van der Waals force increases sharply when the two particles come very close to contact with each other, it becomes very difficult to correctly integrate the equations of motion due to the discrete time interval (see the example of two particle collision in Fig 2.7. In Table 2.1a we show the potential energy ratios between the simulation results and the theoretical integration. Correct integration would give a result of one in the ratio. Obviously, the simulations with smaller time steps will give a more accurate potential energy. However, from the results following in this thesis, we know that it takes a long time for our system to converge to a steady state. It is then impractical to use a time step as small as $10^{-10}$ to carry out our simulations. 


\begin{tabular}{c|c}
\hline$\delta t$ & $U_{\text {sim }} / U$ \\
\hline $10^{-5}$ & $8.059676 \times 10^{-7}$ \\
$10^{-6}$ & $2.056143 \times 10^{-5}$ \\
$10^{-7}$ & $8.061747 \times 10^{-5}$ \\
$10^{-8}$ & $2.046325 \times 10^{-3}$ \\
$10^{-9}$ & $1.420558 \times 10^{-2}$ \\
$10^{-10}$ & $1.437130 \times 10^{-1}$ \\
\hline
\end{tabular}

(a) The original van der Waals force.

\begin{tabular}{c|l}
\hline$\delta t$ & $U_{\text {sim }} / U$ \\
\hline $10^{-5}$ & $9.987851 \times 10^{-1}$ \\
$10^{-6}$ & $9.999584 \times 10^{-1}$ \\
$10^{-7}$ & $9.999878 \times 10^{-1}$ \\
$10^{-8}$ & $9.999996 \times 10^{-1}$ \\
$10^{-9}$ & $9.999999 \times 10^{-1}$ \\
$10^{-10}$ & $1.000000 \times 10^{0}$ \\
\hline
\end{tabular}

(b) The shifted van der Waals force.

Table 2.1: The potential energy ratios between simulation $U_{\text {sim }}$ and theory $U$ change with the simulation time step $\delta t$.

To overcome this problem, we employ a shifted van der Waals force, that reads

$$
\begin{aligned}
& f_{\text {shift }}^{v d w}=-\frac{H_{\text {shift }} d / 2}{(h+d / c)^{2}}, \\
& H_{\text {shift }}=h_{c} H \frac{2(2+c)}{c^{2}},
\end{aligned}
$$

where $h_{c}$ is the near surface cutoff for the force slope before shifting. The Hamaker constant $H$ is modified to $H_{\text {shift }}$, such that the total potential energy is identical with the original one. We can see from Table $2.1 \mathrm{~b}$ that the simulation with the shifted force gives the correct potential energy to a very good approximation already with a time step of $10^{-6} \mathrm{~s}$, which is our choice in the simulations.

\subsection{Observables}

To characterize the states observed in our system, we use the following observables: the radial distribution function $g(r)$, the order parameter $q_{6}$, the coordination number $Z$ and the connection number $\xi$.

\subsubsection{The radial distribution function $g(r)$}

In a system of particles, the radial distribution function (RDF) $g(r)$, also known as pair correlation function, describe how the density varies as a function of distance from a reference particle. It is a measure of the probability to find a particle at distance of $r$ 
away from a reference particle. It is defined as

$$
g(r)=\frac{V}{N^{2}}\left\langle\sum_{i} \sum_{j \neq i}^{N} \delta\left(r-r_{i j}\right)\right\rangle,
$$

where $V$ is the volume of the system, and $\delta(x)$ is the Dirac distribution.

In granular systems, as the filling fraction increases, four typical structures can be observed: loose packing, random close packing, and two crystalline forms, as shown in Fig. 2.8 (89). At $\phi=0.55$, the system is in a loose packing state as the second peak of $g(r)$ at $r=\sqrt{3} d$ shows. $\phi=0.64$ is the maximum filling fraction for randomly packed hard perfect spheres and it is called random close packing (RCP). The second peak at $r=\sqrt{3} d$ will split into two, one at $r=\sqrt{3} d$ and the other one at $r=\sqrt{2} d$. This typical structure is used in the following work to detect if the system exhibits random close packing or not. Above $\phi=0.64$, crystalline structures will arise. At $\phi=0.67, g(r)$ plot shows more tiny peaks, and that is the signal of crystallizing inside the system. Approaching $\phi=0.74$, which is the maximum possible close-packing for equal sized hard spheres, more and more peaks appear.

\subsubsection{The coordination number $Z$}

The average number of nearest neighbors for each particle is known as the coordination number $Z$. Conventionally, the search range for nearest neighbors is limited to the second peak of the $g(r)$ plot. The typical $Z$ number for random close packing of hard spheres is 6 . This number will however be slightly larger than 6 for soft spheres. For a gaseous system $Z$ will be zero, while for a liquid $Z$ is non-zero but smaller than 6 . It is easy to determine the coordination number for crystalline structure, such as $Z=12$ for the hexagonal close packing and face-centered cubic structure. Besides the hexagonal structures, $Z$ will also be used to distinguish the liquid from the gas state.

\subsubsection{The bond orientational order parameters: local $q_{6}$ and global $Q_{6}$}

The $q_{6}$ number is one of a series bond order parameters introduced by Paul J. Steinhardt et al. (90) . They are sensitive to the differences between the various crystalline structures and insensitive to the orientation of the crystal in space. In a liquid they will vanish rapidly but they are large enough in the solid. 


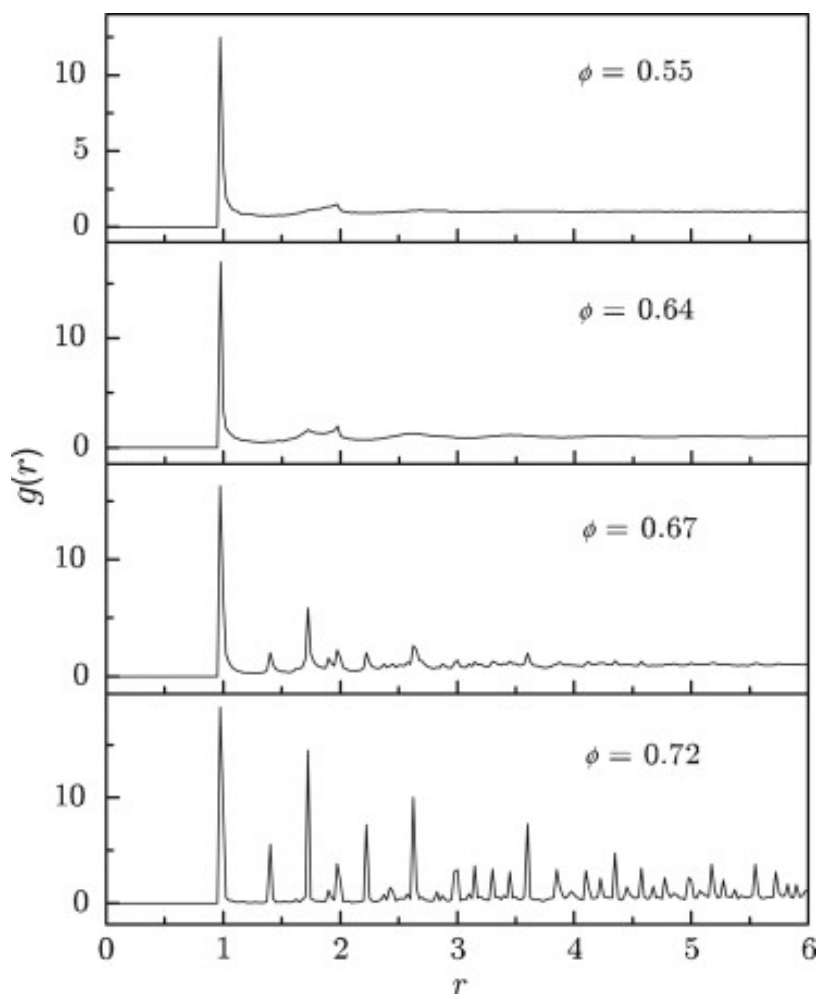

Figure 2.8: The radial distribution function $g(r)$ for different average filling fractions $\phi$ Reprinted from (89). 
To measure the bond orientational order parameters (91), one first needs to find the list of neighbors of each particle, which consists of all particles within a radial distance $r_{c}$ of each particle. The local structure around one particle $i$ is characterized by the set of numbers

$$
q_{l, m}(i)=\frac{1}{N_{b}(i)} \sum_{j=1}^{N_{b}(i)} Y_{l, m}\left(\hat{\mathbf{r}}_{i, j}\right),
$$

where $\mathbf{r}_{i, j}$ is the distance vector between particle $i$ and one of its neighbors $j$. The unit vector $\hat{\mathbf{r}}_{i, j}$ uniquely determines the polar and azimuthal angles $\theta(i)$ and $\phi_{i, j}$, which are needed to calculate the spherical harmonics $Y_{l, m}$.

The number $q_{l, m}$ is however dependent on the reference frame used. A reference-invariant quantity associated to $i$ can be defined as

$$
q_{l}(i)=\left(\frac{4 \pi}{2 l+1} \sum_{m=-l}^{l}\left|q_{l, m}(i)\right|^{2}\right)^{1 / 2}
$$

By averaging $q_{l, m}(i)$ over all the particles in the system, we can obtain a global order parameter $Q_{l, m}$.

$$
Q_{l, m}=\frac{\sum_{i=1}^{N} N_{b}(i) q_{l, m}(i)}{\sum_{i=1}^{N} N_{b}(i)} .
$$

Again, $Q_{l, m}$ depends on the reference frame. Rotationally invariant combinations can be constructed as

$$
Q_{l}=\left(\frac{4 \pi}{2 l+1} \sum_{m=-l}^{l}\left|Q_{l, m}\right|^{2}\right)^{1 / 2}
$$

This set of bond order parameters include $Q_{4}, Q_{6}, \widehat{W}_{4}, \widehat{W}_{6}$. In Table. 2.2 we show the typical values of these parameters for some specific geometries. For our system, which turns out to have local hexagonal order, $Q_{6}$ is the best choice.

As examples we show in Fig. 2.9 the calculation of $q_{6}$ for some ordered arrangements of particles; they are in perfect match with the numbers in Table. 2.2 For simple cubic, hexagonal close packing and face-centered cubic, there is no difference between the local order parameter $q_{6}$ and the global $Q_{6}$. For body-centered cubic, the local $q_{6}$ number varies with the neighbor cutoff $r_{c}$, and it is equal to the global $Q_{6}$ number only for $r_{c} \geq 1.5 d$. 
Table 2.2: Bond orientational order parameters for geometries. Reproduced the data from (91)

\begin{tabular}{lllll}
\hline \hline & $Q_{4}$ & $Q_{6}$ & $\widehat{W}_{4}$ & $\widehat{W}_{6}$ \\
\hline fcc & 0.191 & 0.575 & -0.159 & -0.013 \\
hcp & 0.097 & 0.485 & 0.134 & -0.012 \\
bcc & 0.036 & 0.511 & 0.159 & 0.013 \\
sc & 0.764 & 0.354 & 0.159 & 0.013 \\
Icosahedral & 0 & 0.663 & 0 & -0.170 \\
liquid & 0 & 0 & 0 & 0 \\
\hline
\end{tabular}

\subsubsection{The connection number $\xi$}

The connection number $\xi(\underline{92})$ is a quantity based on the bond orientational order parameter $q_{l, m}(i)$ and used to differentiate between liquid-like and solid-like particles. Furthermore, it is used to identify solid clusters. For one particle $i, \xi$ is defined as

$$
\xi(i)=\sum_{j=1}^{N_{b}(i)} H\left(d_{l}(i, j)-d_{c}\right)
$$

where $H$ is the Heaviside step function and

$$
d_{l}(i, j)=\frac{\sum_{m=-l}^{l} q_{l, m}(i) q_{l, m}^{*}(j)}{\left(\sum_{m=-l}^{l}\left|q_{l, m}(i)\right|^{2}\right)^{1 / 2}\left(\sum_{m=-l}^{l}\left|q_{l, m}(j)\right|^{2}\right)^{1 / 2}}
$$

and $d_{c}$ is a threshold value. Solid-like particles are identified as particles with connection number $\xi \leq \xi_{c}$. A cluster consists of solid-like particles with at least one solid-like neighbor in the same cluster.

The measurement of the connection number $\xi$ relies on three parameters: the neighbor list cutoff $r_{c}$, the dot-product cutoff $d_{c}$ and the index for the bond orientational order parameter $l$. For our system, we choose $r_{c}=1.01 d, d_{c}=0.8$ and $l=6$. Additionally, $\xi_{c}=6$ is chosen to identify the solid-like particles. 


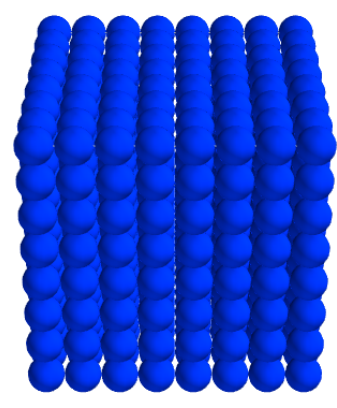

(a) Simple cubic.

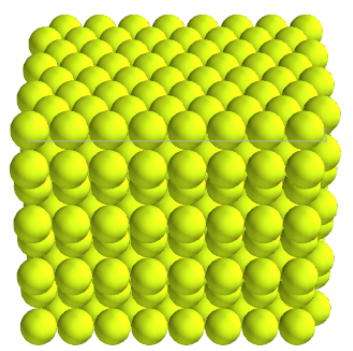

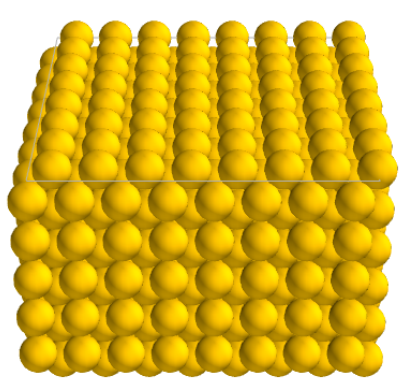

(b) Body-centered cubic.

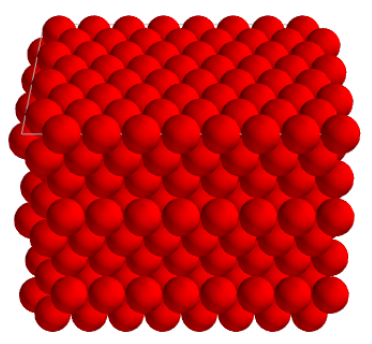

(d) Face-centered cubic.

(c) Hexagonal close packing.

$$
q_{6}
$$

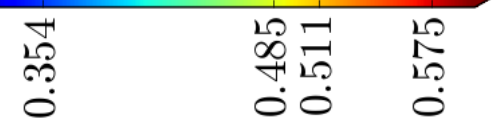

Figure 2.9: The calculations of the local order parameter $q_{6}$ for four regular packings of identical spheres. Particles colors correspond to the local $q_{6}$ number.

\subsection{Conclusion}

In this Chapter, we have explained the details of our time-driven MD algorithm. The force models for the included interactions, inelastic collision and van der Waals interaction are explained in detail. We have also discussed the basic structure of the algorithm, together with the details of methods to search neighbors and propagate particles. At the end of 
this Chapter, we have defined the system parameters and observables used to characterize the observed states. 



\section{System with vdW interaction}

\subsection{Collisions between two particles with van der Waals interactions}

Two granular particles without any cohesive interaction will fly away from each other after a collision. With van der Waals interaction, when the total kinetic energy after collision $E_{k i n}^{a}$ is smaller than the van der Waals potential energy $U_{v d w}$, two particles will be trapped inside the potential well. They will bounce back and forth inside the well, till all the energy is dissipated. The final state is the two particles are rest at contact with each other but with no overlap. In Fig. 3.1. we show a diagrammatic sketch of a pair collision, together with the plots of the velocity of one particle, the mutual distance, and the acceleration of one particle for two cases with different initial velocities. The first case is with $E_{k i n}^{a}<U_{v d w}$ shown in Fig. 3.1b and the other one is with $E_{k i n}^{a}>U_{v d w}$ shown in Fig. $3.1 \mathrm{c}$.

\subsection{The system without van der Waals interaction}

To fully understand the influence of van der Waals interactions in a granular system, we first need to understand the system without van der Waals interactions. The work reported by K. Roeller et al. in 2010 (49) studied a confined granular gas system driven by sinusoidally vibrating walls. They varied the filling fraction and driving amplitude, and found a liquid-gas phase coexistence region (shown in Fig. 3.2. We performed similar simulations and sparsely mapped the same phase spaces for the system without van der Waals interaction, and furthermore checked how this coexistence region vary with the dissipation levels. In Fig. 3.3 we show the phase diagrams for $\epsilon=0.6,0.7,0.8$ and 0.9 . We obtain the same coexistence regions (the region marked with red dots). the coexistence region will shrink as $\epsilon$ increases and approaches 1 . Beside the coexistence state, we also 


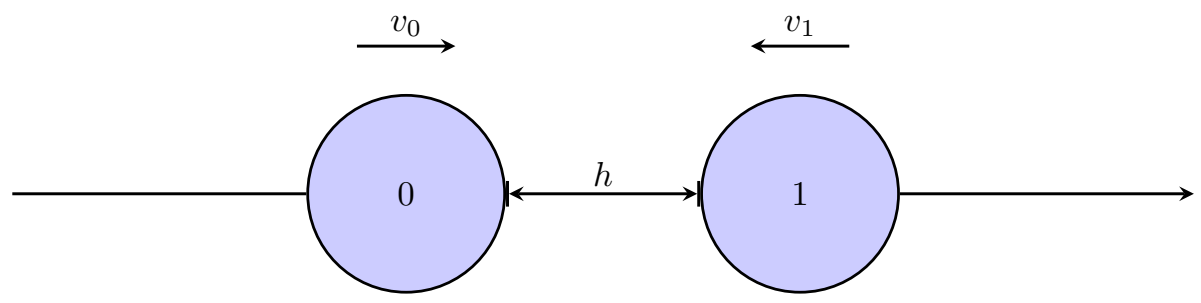

(a)

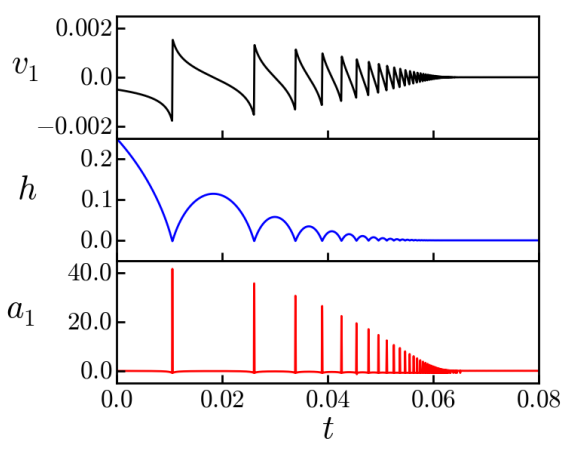

(b)

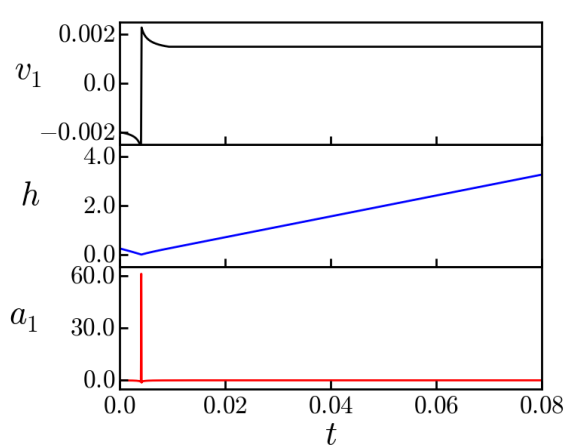

(c)

Figure 3.1: (a) The diagrammatic sketch for pair collision. (b) The case for two particles trap each other after collision. (c) The case for two particles fly away from each other after collision. 
find a state characterized by waves traveling through the system (marked as blue dots in the phase diagrams), where two density peaks keep propagating through the system in the opposite directions perpendicular to the walls. The filling fraction distribution in $x$-direction and the the histogram of local filling fraction for steady states of three selected parameter groups are shown in Fig. 3.4. which are respectively for the homogeneous state, the coexistence state and the traveling wave. In the coexistence state, the denser part will became denser and denser as $\epsilon$ decreases, but never reach the value 0.64 where the solid-like state begins.

\subsection{Theoretical prediction}

Before we start to explore the phase space, we first try to predict in which region of the phase diagram the van der Waals interaction may become relevant. We suppose that region will lie where the van der Waals potential energy is comparable with the granular temperature of the non-cohesive granular system.

For a system confined by walls, the collisions frequency with walls, $f_{\text {wall }}$, is related to the pair collision rate $f_{\text {coll }}$ in the system via

$$
f_{\text {wall }}=\frac{2 l_{m}}{h} f_{\text {coll }}=2 f_{\text {coll }} K_{n}
$$

where $l_{m}=R / 6 \bar{\phi}$ is the mean free path of particle, and $K_{n}=l_{m} / L$ is the Knudsen number of granular gas in a box with height $L$. The energy injection rate (i.e. the energy injected per unit time) $e_{i n j}$ is

$$
e_{i n j}=f_{w a l l} E_{v i b}
$$

According to kinetic theory, the energy dissipation rate $e_{\text {diss }}$ in a gas-like granular system is

$$
e_{\text {diss }}=f_{\text {coll }}\left(1-\epsilon^{2}\right) T_{g}
$$

where $T_{g}$ is the granular temperature. In the steady state, the total energy of the system should be dynamically balanced, i.e. $e_{\text {diss }}=e_{i n j}$. Then, we can obtain the granular temperature as (64)

$$
T_{g}=\frac{2 K_{n} E_{v i b}}{1-\epsilon^{2}}
$$

The van der Waals potential energy $U_{v d w}$ is found by integrating the van der Waals force 
based on the Hamaker theory, with the near surface cutoff $h_{c u t}=10^{-10}$ and the minimum force truncation at $h=0.25 d$

$$
U_{v d w}=10^{10} \frac{H R}{6} .
$$

We define a number $r_{e}$ as the ratio of the two energy scales, granular temperature $T_{g}$ and $U_{v d w}$. We assume the vibration energy $E_{v i b}=\frac{1}{2} m(A \omega)^{2}$ which is the maximum energy one particle can attain after a collision with the wall. Then

$$
\begin{aligned}
r_{e} & =\frac{T_{g}}{U_{v d w}}=C_{0} \frac{A^{2} R^{3}}{\phi}, \\
C_{0} & =\frac{4.0 \times 10^{-10} \pi \rho \omega^{2}}{3.0 L\left(1-\epsilon^{2}\right) H}
\end{aligned}
$$

In our system, $C_{0}$ is a constant, with all the parameters $\rho, \omega, L, \epsilon$ and $H$ fixed. In Fig. 3.5. we show the contour plots of $\ln \left(r_{e}\right)$ in the space of $A / d$ and $\bar{\phi}$ for different particle sizes. We could see that there is a region in the phase spaces where the two energy scales are comparable (with $-1.0 \leq \ln \left(r_{e}\right) \leq 1.0$ ), and it will expand and shift towards a regime of stronger driving as the particle diameter decreases. That is reasonable as the van der Waals interaction plays a more and more important role when the particle size grows smaller. When the particle diameter is above $100 \mu m$, the van der Waals potential energy becomes negligible (see Fig. 3.5f). Keeping in mind considerations of computational feasibility of our simulations, we choose to perform simulations for a system composed of particles with $d=70 \mu \mathrm{m}$.

\subsection{Simulation details}

We mapped the phase space where the van der Waals potential energy is comparable with the granular temperature. There, we find a new coexistence state (one snapshot thereof is shown in Fig. 3.6. The denser part appears to be solid-like. In the following chapters we will carefully characterize this phase.

\subsubsection{Energy convergence}

Compared to simulations for a system without van der Waals interaction, it takes a much longer time to reach a steady state for the system with van der Waals interaction. In Fig. 3.7 we show how the kinetic energy changes with simulation time for both cases, using 
the same filling fraction and driving amplitude. Actually, the system does not converge to a steady state where the kinetic energy reaches a constant value. The interesting phases (dense packing or crystals) start to appear after $10^{7}$ simulation time steps when the kinetic energy starts to show small scale oscillations. So, all statistical averages should be performed afterwards.

\subsubsection{Hysteresis}

Because of the cohesive forces when the van der Waals interactions are considered, the system exhibits hysteretic effects, that is, it retains a memory of its history. These effects make it difficult to establish the boundary of the coexistence state. Especially near the metastable regime between the binodal curve and the spinodal curve, the system prefers evolving to the coexistence state when the initial configuration is not carefully prepared in a homogeneous state. For some of the simulations, mostly with relatively high filling fractions, two kinds of simulations with different initial conditions are needed to decide what is the real steady state. The first type o initial condition is one where all particles are randomly arranged in the box, and that is the normal way we initialize our system. The second type is one where we start from a homogeneous steady state previously simulated at the same filling fraction but with a higher driving amplitude. If the final states we obtain from these two types of initial conditions are different, this state point is classified as belonging to the metastable regime.

\subsubsection{Stabilization of the phases}

In the solid-gas coexistence regime, some driving amplitudes could make the system crystallize. However, in some cases, the solid plug oscillates up and down between two walls (shown in Fig. 3.8a). The crystal structure may break or even disintegrate after several impacts with the walls. It is not clear why this sometimes happens but it needs to be monitored otherwise statistical averages may show spurious effects. when such a situation was detected, we performed additional simulations with the same driving and filling parameters but longer simulation box. We find that the case is improved at $x=480 d$, where the solid plug has a vanishing average velocity in the $z$ direction (see Fig. $3.8 \mathrm{~b}$. However, it oscillates even more for a system with $x=640 d$ (see Fig. 3.8c). Obviously, increasing the system size does not improve the situation, so this problem is not introduced by finite size effects. For this kind of state points in the phase diagram, we need to redo the simulations with special care to the initial configuration. One choice is to initialize with a 
non-oscillating steady-state configuration from a different simulation with the same filling fraction but different amplitude. A second method is to initialize the system with the crystalline structure we observe as being stable in the neighborhood of the phase diagram under consideration.

\subsection{Conclusion}

In this chapter, we reproduced the phase diagrams for the sinusoidally driven granular system without van der Waals interactions, as a reference for the following study about the impact of van der Waals interactions. Instead of focusing on one restitution coefficient, we also explored how the phase regimes vary with different dissipation levels and we found a new traveling wave regime at relatively strong dissipated system. Before exploring the phase space for a system with van der Waals interactions, we theoretically predicted the region where the van der Waals interaction may play a role. And indeed, we found an interesting new coexistence phase in that region. Finally, we explained several difficulties we met during the simulations of the cohesive system and show how we tackled them. 


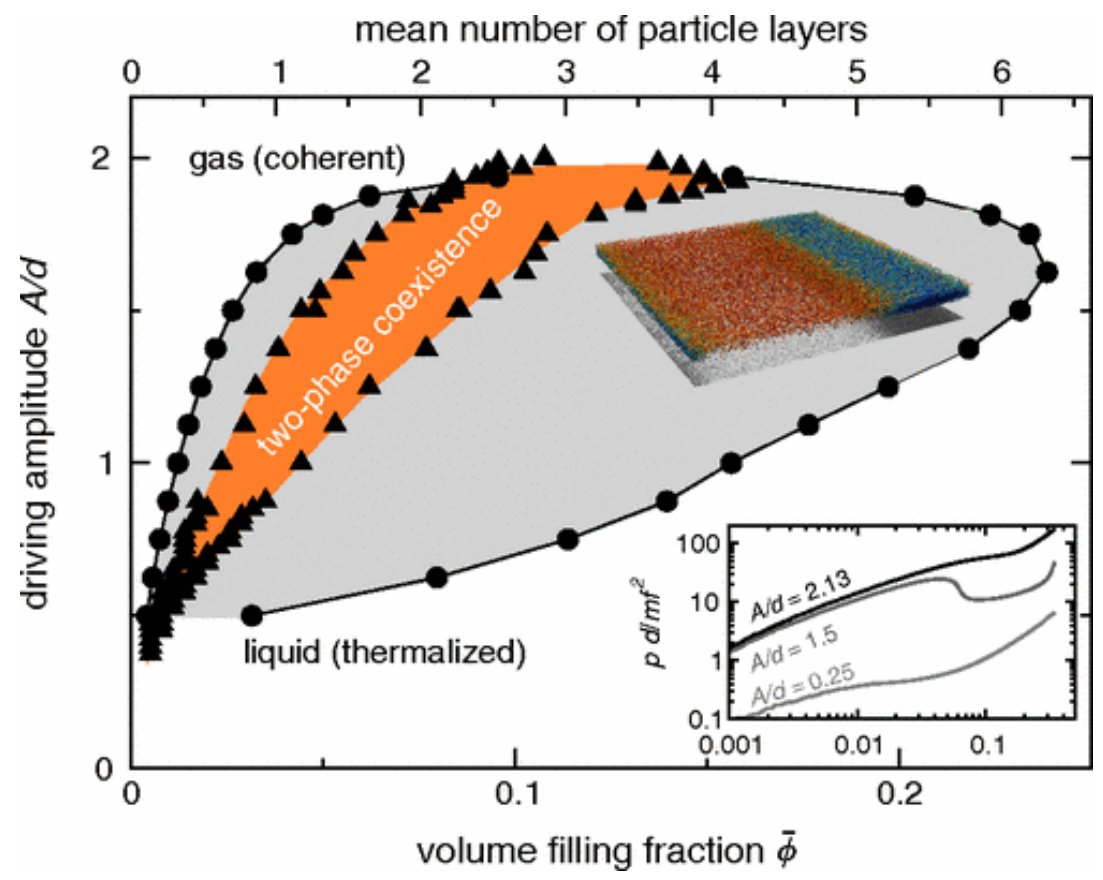

Figure 3.2: The phase diagram in the space of $A / d$ and $\bar{\phi}$, and with $\epsilon=0.9$. The black triangles delimit a region (orange shaded) where the phase separation occurs. The black circle form the "binodal line". The colored inset is one snapshot for the coexistent state. The denser part is blue and the dilute part is orange. Reprinted from (49). 


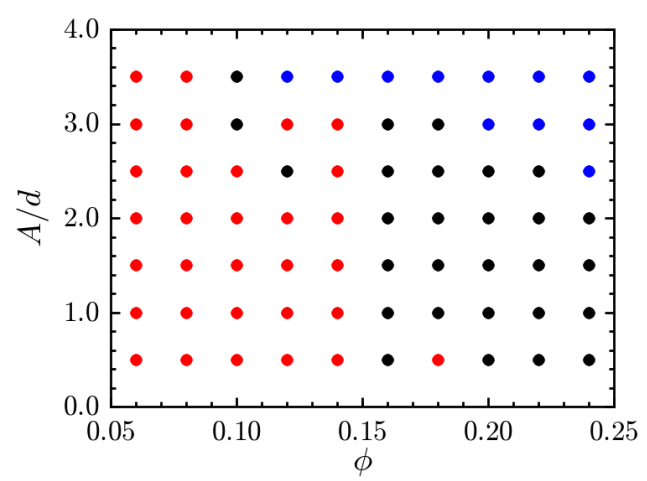

(a) $\epsilon=0.60$

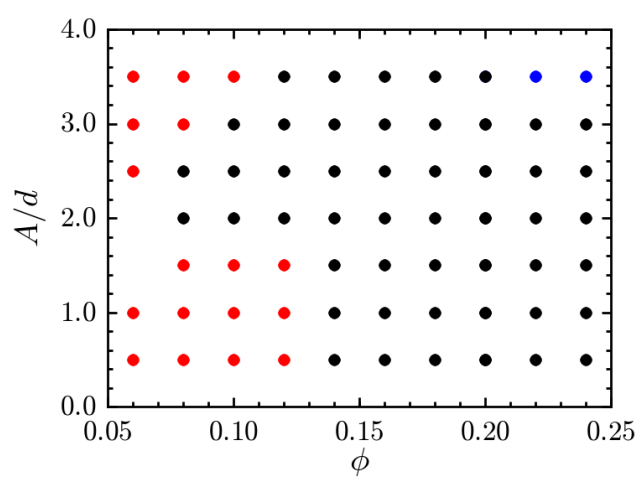

(c) $\epsilon=0.80$

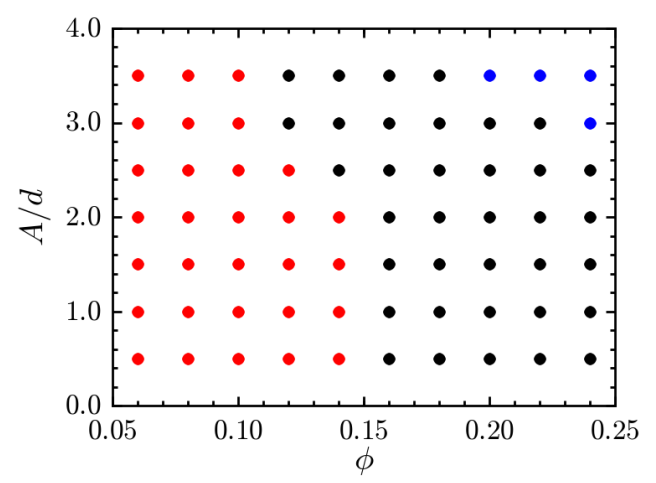

(b) $\epsilon=0.70$

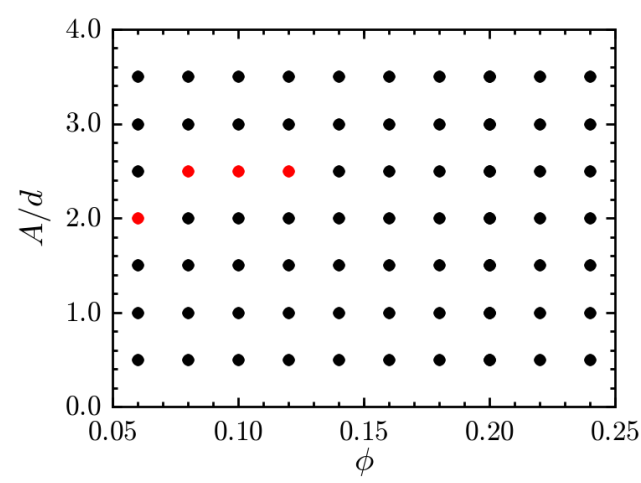

(d) $\epsilon=0.90$

Figure 3.3: Phase diagram for system without van der Waals interaction for different restitution coefficients. The red, black and blue dots mark a coexistence state, $x$-direction homogeneous state, and traveling waves state, respectively. 

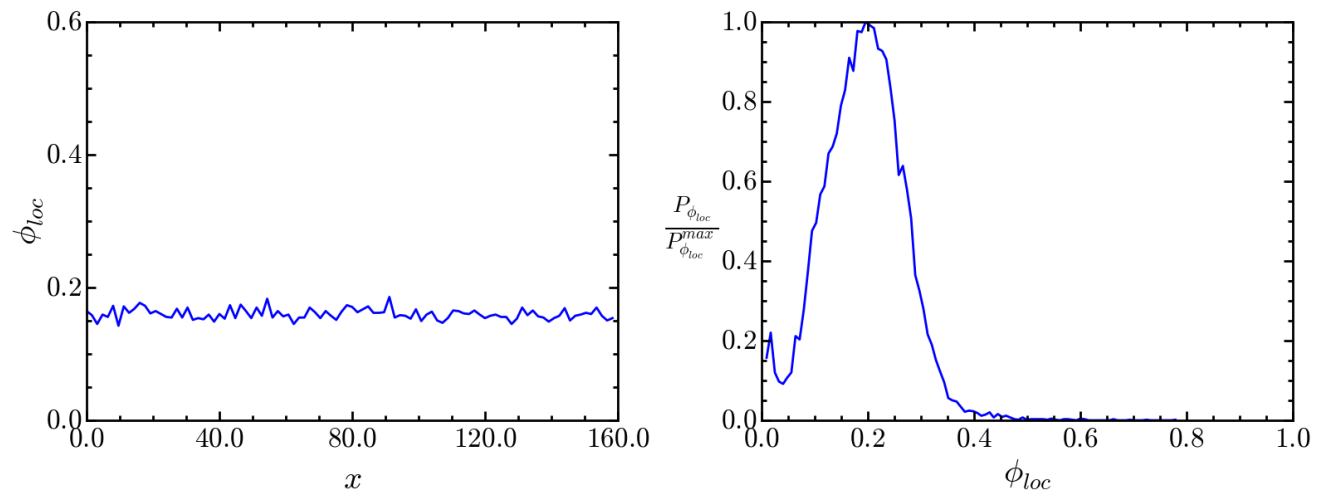

(a) $\epsilon=0.90, A=2.0 d, \phi=0.16$
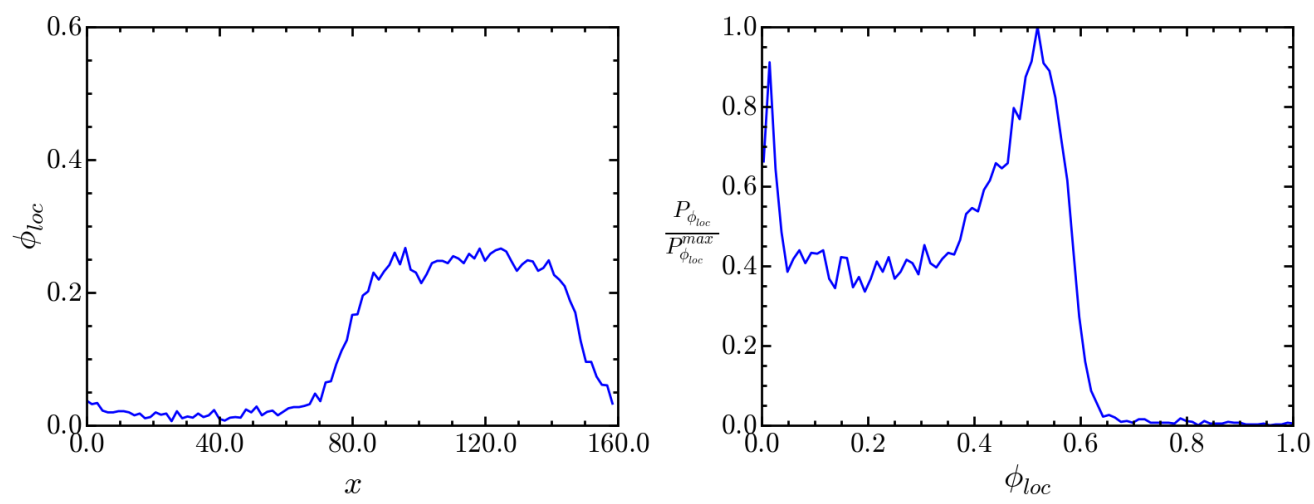

(b) $\epsilon=0.70, A=2.5 d, \phi=0.12$
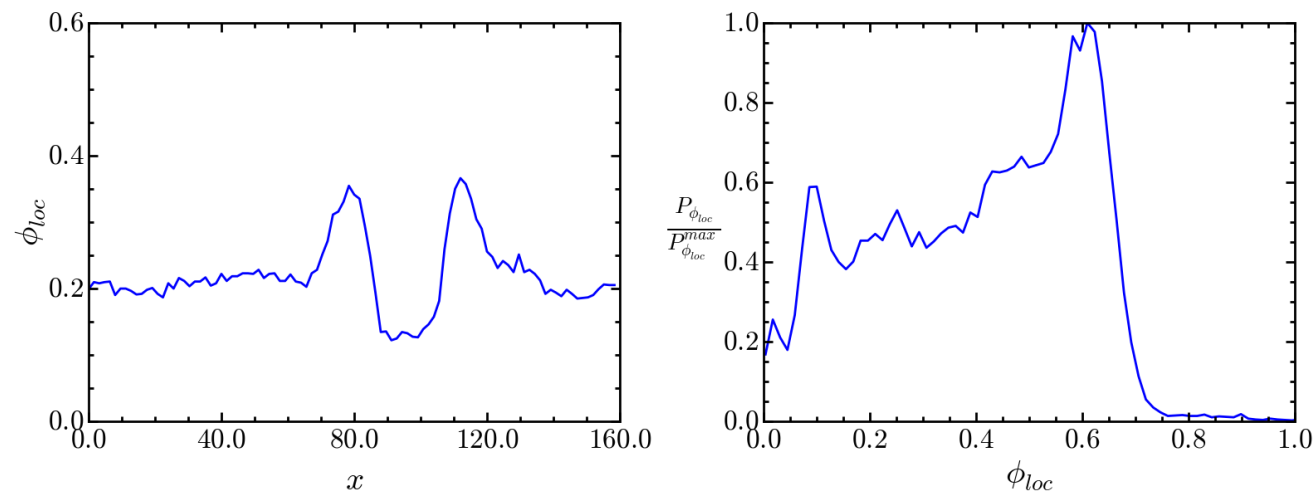

(c) $\epsilon=0.70, A=3.5 d, \phi=0.22$

Figure 3.4: The local filling fraction profile in the $x$-direction (left column) and the the histogram of local filling fraction (right column) for three representative systems exhibiting (a) a homogeneous state, (b) coexisting phases, and (c) traveling wave. 


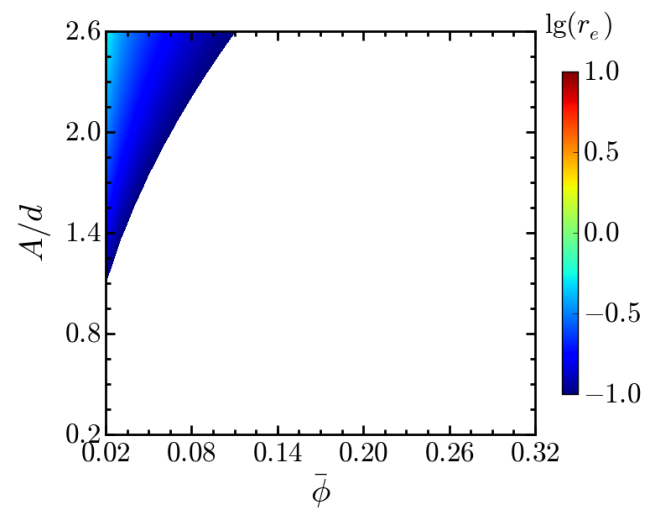

(a) $d=10 \mu m$

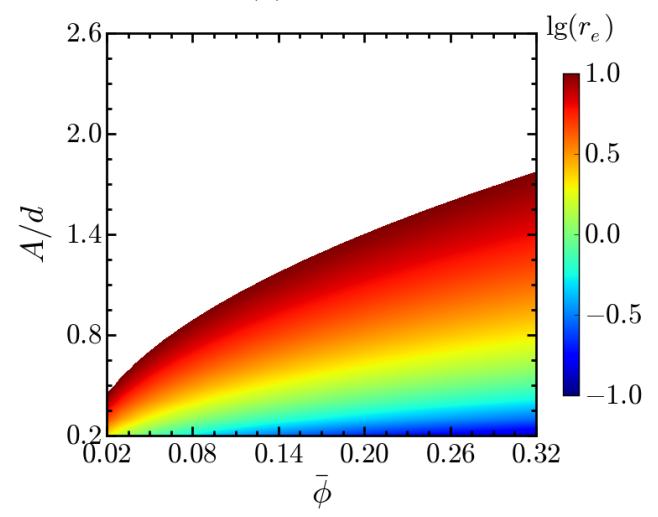

(c) $d=50 \mu m$

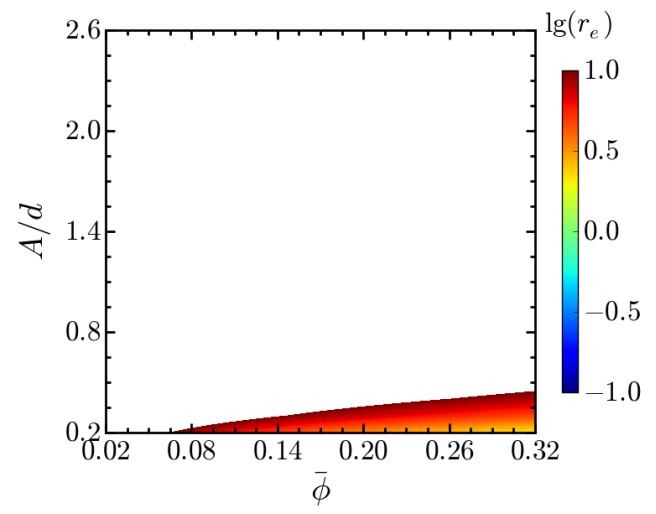

(e) $d=100 \mu m$

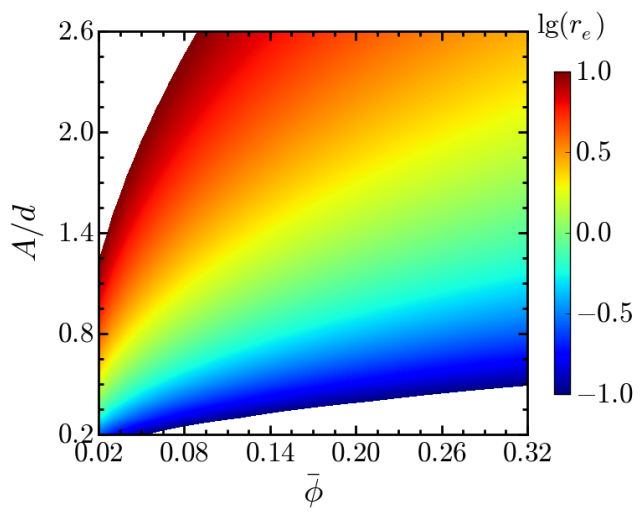

(b) $d=30 \mu m$

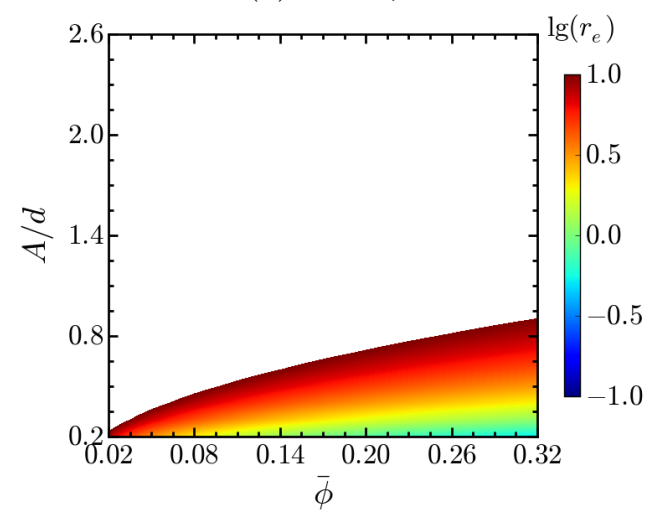

(d) $d=70 \mu m$

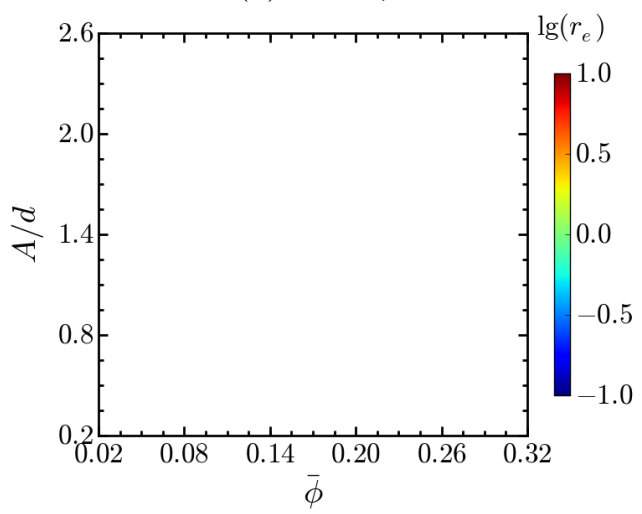

(f) $d=200 \mu m$

Figure 3.5: The contour plots of the energy-scale ratio $r_{e}$ of the granular temperature to van der Waals interaction for different particle sizes. Only the regions where $-1.0 \leq \ln \left(r_{e}\right) \leq 1.0$ are shown in the plots. 


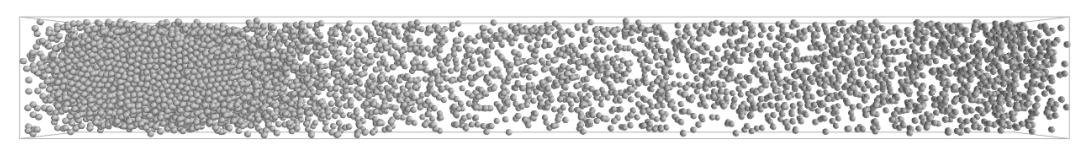

Figure 3.6: Snapshot of a system exhibiting the solid-gas coexistence state with $A=$ $0.46 d, \phi=0.12$.

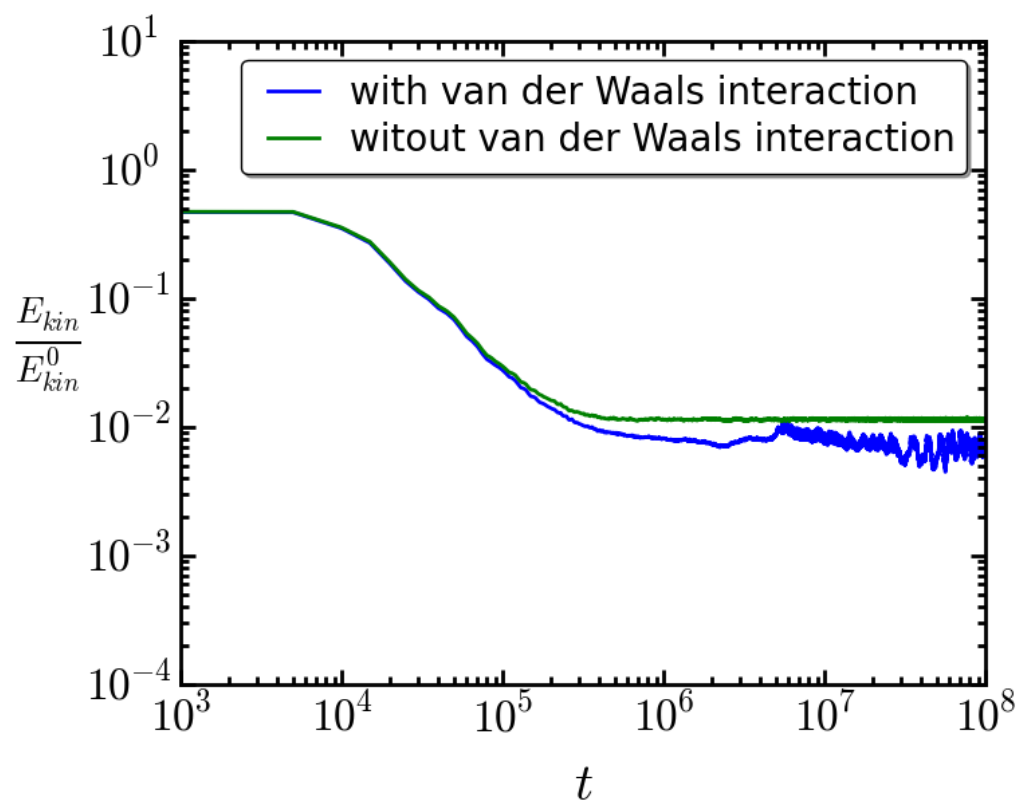

Figure 3.7: Comparison of the temporal evolution of the kinetic energy between a system with van der Waals interactions and without them. Much longer simulation times are necessary when van der Waals interactions are present. 


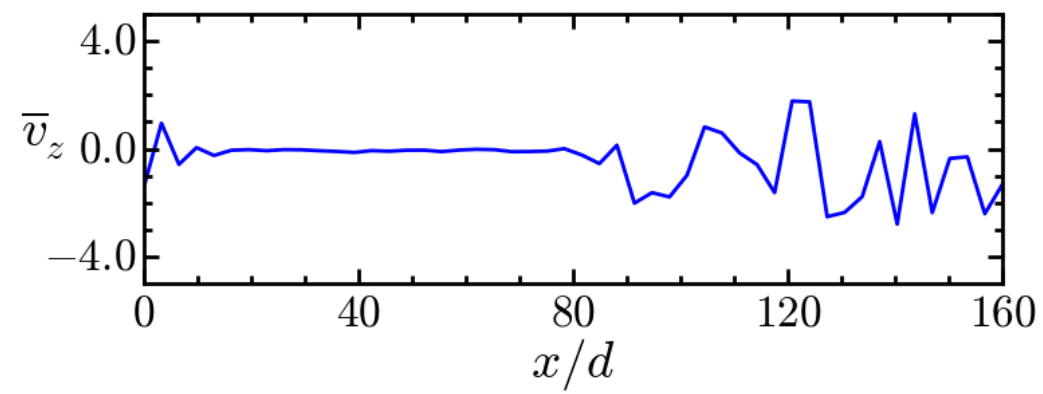

(a) The system with $x=160 d$. The gas part is from $80 d$ to $160 d$ and the rest is solid.

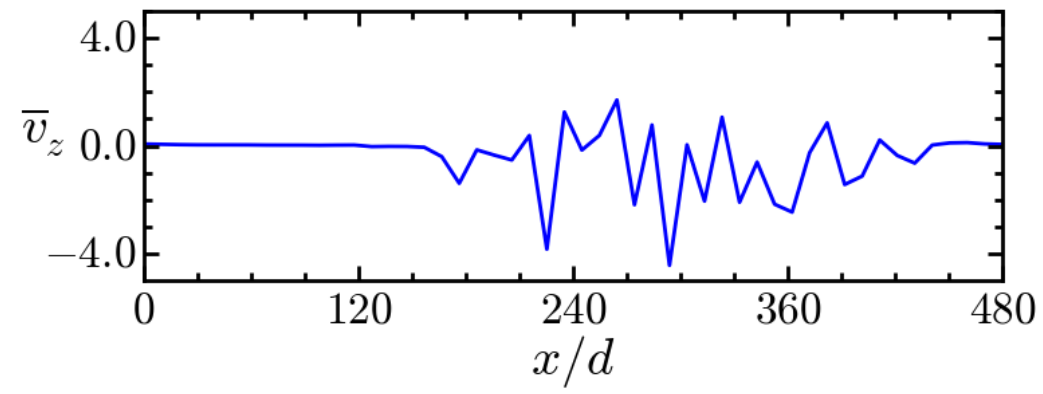

(b) The system with $x=480 d$. The gas part is from $150 d$ to $450 d$ and the rest is solid.

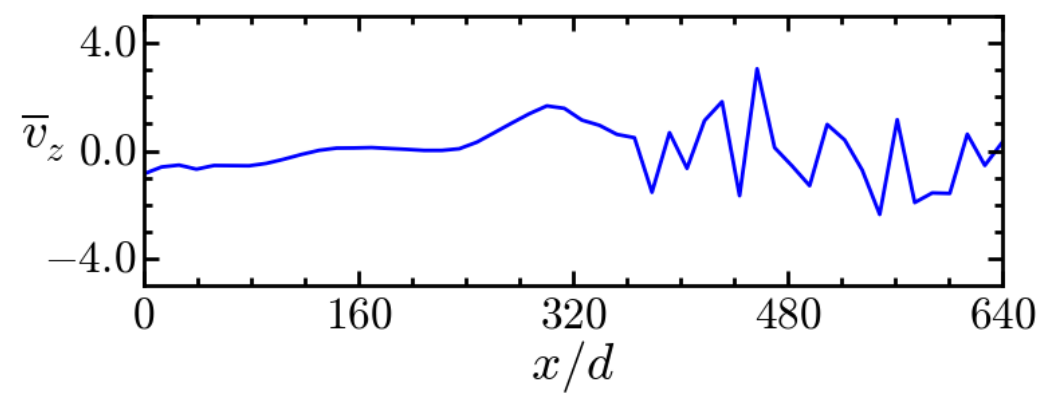

(c) The system with $x=640 d$. The gas part is from $360 d$ to $640 d$ and the rest is solid.

Figure 3.8: Dependence of the average $z$-direction velocity on the $x$ direction for the solidgas coexistence state. Different system lengths are shown. 


\section{Phase diagram}

According to the theoretical prediction discussed in Chap. 2, we explored the region where the van der Waals potential energy is comparable with the granular temperature. That is where $\ln r_{e}$ ranges from -1 to 1 in the averaged filling fraction $\bar{\phi}$ and driving amplitude $A$ space. In this Chapter, we show the phase diagram together with representative snapshots, characteristic of all states observed in the explored region. Further calculations to characterize different phases are shown afterwards.

\subsection{Phase diagram with Snapshots}

In Fig. 4.1, we show the phase diagram obtained from our simulation results. The black filled triangles form the "spinodal line", which delimits the region where the homogeneously initialized system will separate along the $x$-direction into a dense, solid like region and a dilute, gas-like one. The denser part shows poly-crystallization in the orange shaded region and random close packing in the aqua region. The black $\mathrm{x}$-marks are the specific simulations at the boundary of the poly-crystal and gas coexistence (PC-G) state and random close packing and gas coexistence (RCP-G) state, and the black dashed line is the fitting of the boundary line. Outside the coexistence regime, at low amplitude $A$, the energy injection into the system is too weak to overcome the combined effect of attractive van der Waals interactions and the energy dissipation during collisions, so all particles stick together. We call it a "clustered state". The cluster state will turn to translationally invariant gas state as $A$ grows at small $\bar{\phi}$, and turns to homogeneous liquid as $A$ grows at large $\bar{\phi}$. In Fig. 4.2 we show the snapshots for all the possible states.

Apart from the coexistence regime, the clustered states are not always the same. In Fig. 4.3 we show several snapshots of different clustered states with the same averaged filling fraction but different driving amplitudes. When the driving is relative weak, the cluster extends through the whole simulation domain along the $x$ direction. They continuously grow more compact as the amplitude $A$ increases. At the transition to RCP-G, some 


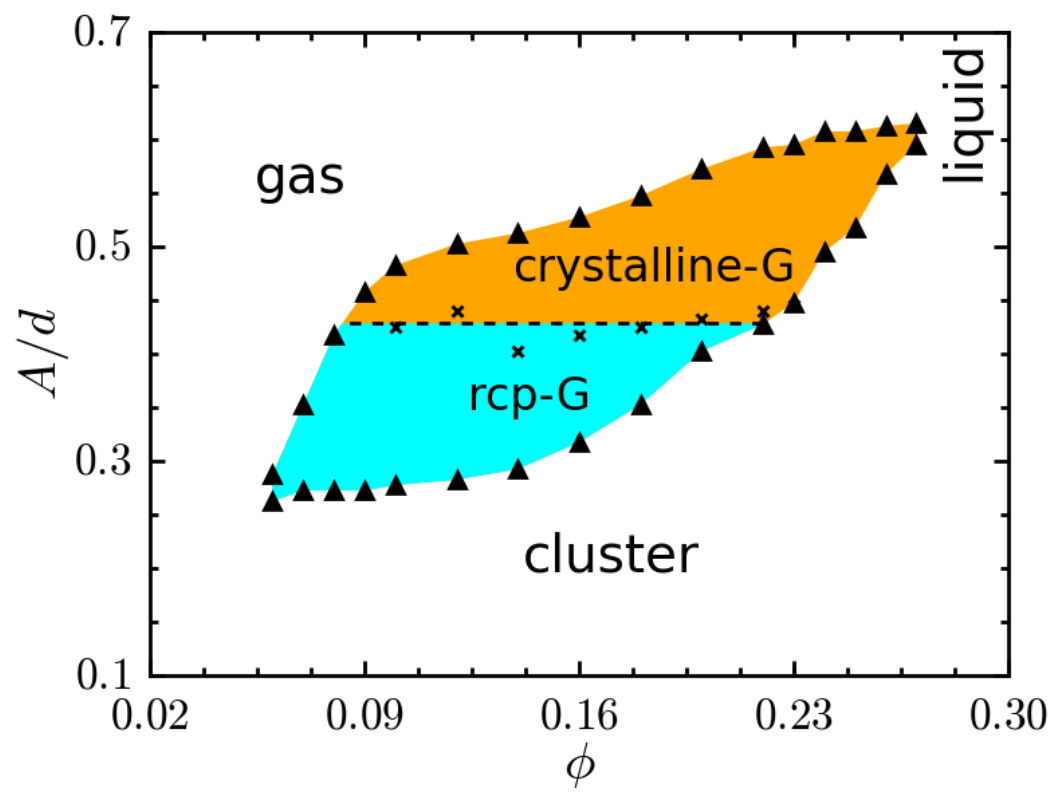

Figure 4.1: The phase diagram in the space of $A / d$ and $\bar{\phi}$ for system with van der Waals interaction and $\epsilon=0.9$.
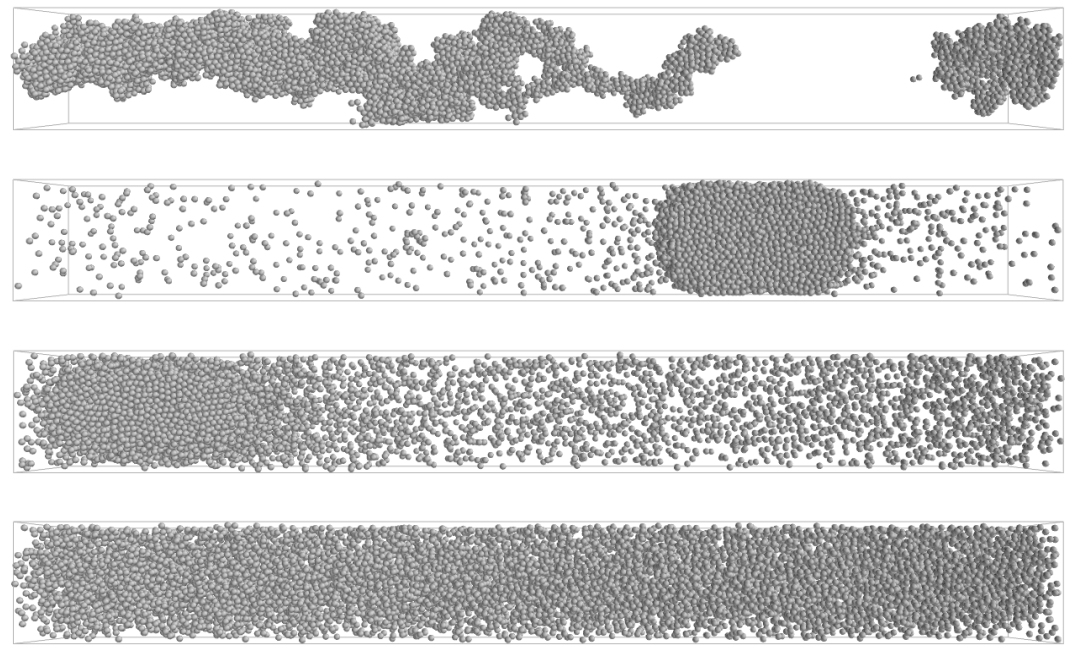

Figure 4.2: The snapshots for system with $\bar{\phi}=0.10$ and $A$ from top to bottom $0.10 \mathrm{~d}$, $0.32 \mathrm{~d}, 0.46 \mathrm{~d}$ and $0.50 \mathrm{~d}$, and they are respectively cluster, RCP-G state, PC$\mathrm{G}$ state and homogeneous state. The system is viewed laterally, while the vibration is imposed in the vertical direction. 
particles escape from the solid-like plug to form a gas, and the system shows coexistence state.
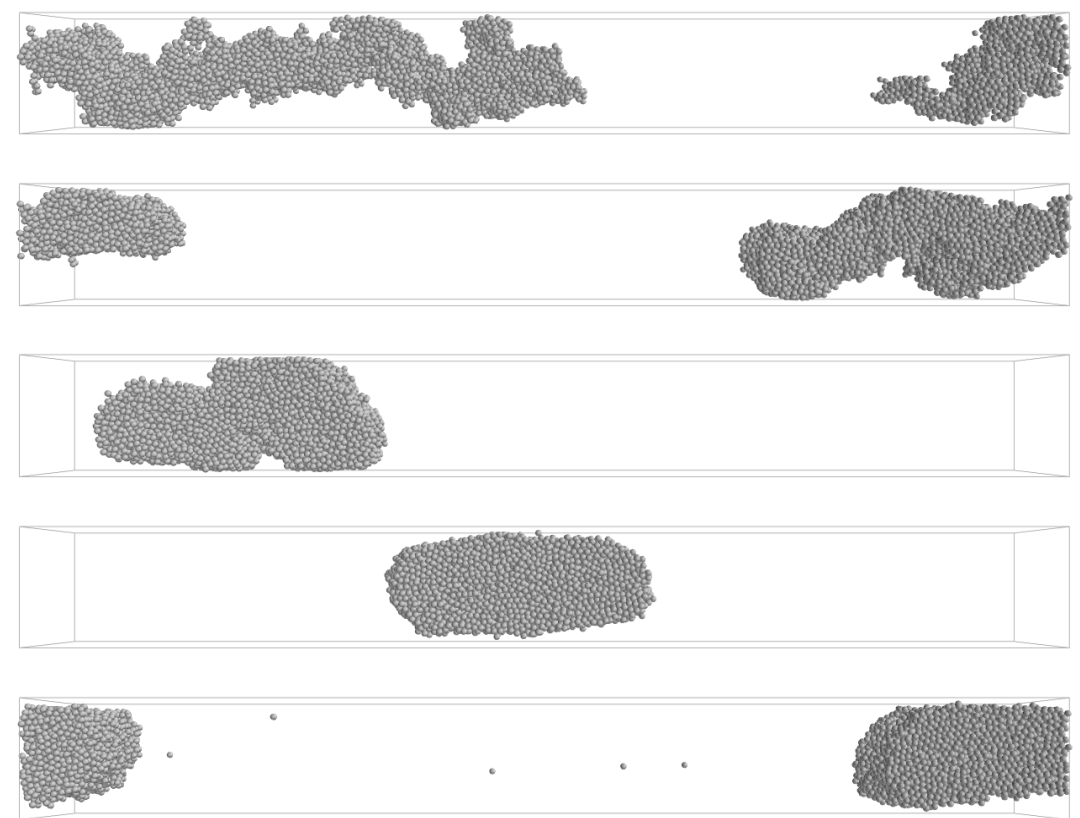

Figure 4.3: The clustered state snapshots with $\bar{\phi}=0.10$ and $A$ from top to bottom $0.10 \mathrm{~d}$, $0.20 \mathrm{~d}, 0.24 \mathrm{~d}, 0.26 \mathrm{~d}, 0.28 \mathrm{~d}$.

\subsection{Characterization of phases}

\subsubsection{Local filling fraction}

The local filling fraction $\phi_{l o c}$ is measured for all different states using Voronoi tessellation. For each snapshot, we calculate the local filling fraction $\phi_{l o c}$ for all particles from their own Voronoi volumes, then compute the probability distribution of the $\phi_{l o c}$ by averaging over 20 independent, steady-state snapshots for each simulation. The maximum of the distribution $\phi_{p_{\max }}$ is chosen to mark the state of the system.

In Fig. 4.4, we show how the $\phi_{p_{\max }}$ varies with the driving amplitude $A$ for different averaged filling fractions $\bar{\phi}$. Two typical $\phi_{p_{\max }}$ can be seen in the plot. One is around 0.64 which is the filling fraction for random close packing, the other one is 0.74 which is the filling fraction for hcp and fcc structures. 


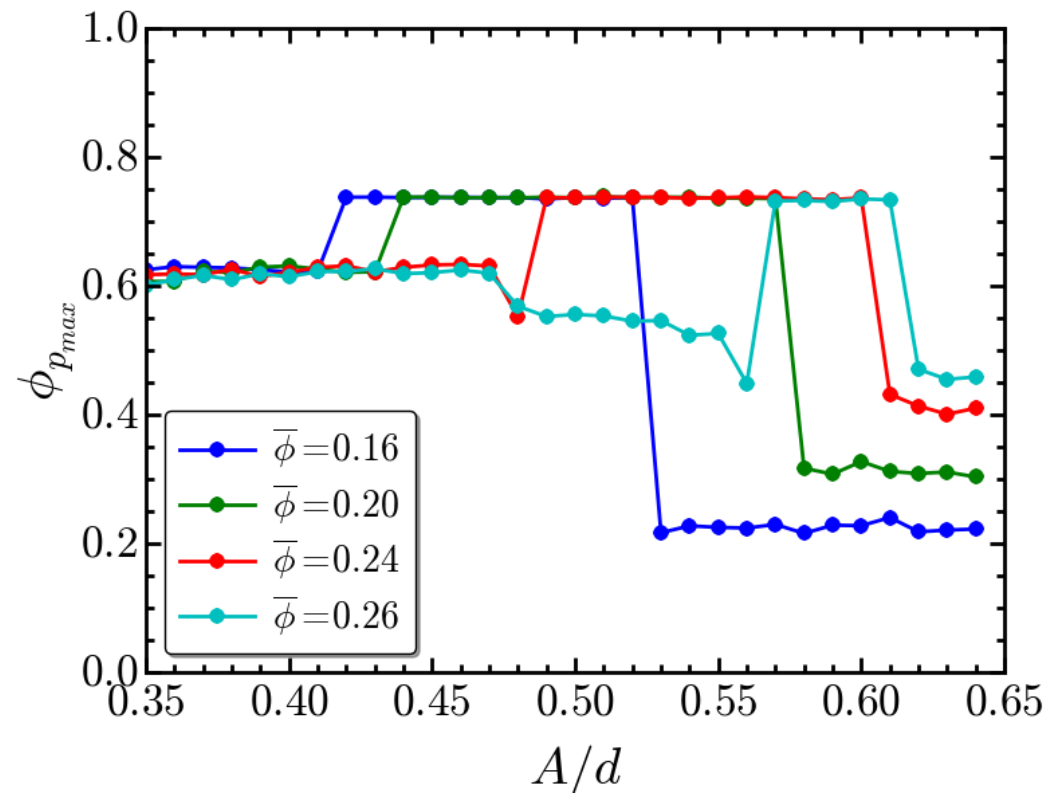

Figure 4.4: The $\phi_{p_{\max }}$ change with the $A / d$ for different $\bar{\phi}$.

\subsubsection{RDF}

In Fig. 4.5, we shows the radial distribution function (RDF) $g(r)$ plots for different $\bar{\phi}$. For each $\bar{\phi}$, we only picked three plots with different $A$ which represent different states.

For $\bar{\phi}=0.16$ (shown in Fig. $4.5 \mathrm{a}$ ), the $g(r)$ plot shows the typical structure of random close packing at $A=0.40 d$. More peaks arise at $A=0.50 d$ which shows that crystallization occurs in the system at this driving amplitude. Above the coexistence regime, at $A=$ $0.60 d$, only one peak at $r=d$ appears and that is the homogeneous gas state.

For $\bar{\phi}=0.20$ (shown in Fig. 4.5b), at $A=0.36$, which is at the clustered regime, the $g(r)$ plot shows the typical split second peak of $g(r)$ that characterizes random close packing. More peaks arise at $A=0.50 d$ which shows that a crystal structure emerges in the system. At $A=0.42 d$, near the boundary line between PC-G state and RCP-G state, small peaks starts to arise on the basis of the random close packing $g(r)$ structure.

For $\bar{\phi}=0.23$ (shown in Fig. 4.5c), the case is rather similar to the $\bar{\phi}=0.16$ case and it shows signals of RCP at $A=0.40 d$ and crystallizing at $A=0.50 d$. Another small peak starts to grow at $A=0.60 d$ and it says that the homogeneous state above the coexistence regime for this filling fraction is liquid state rather than gas. 

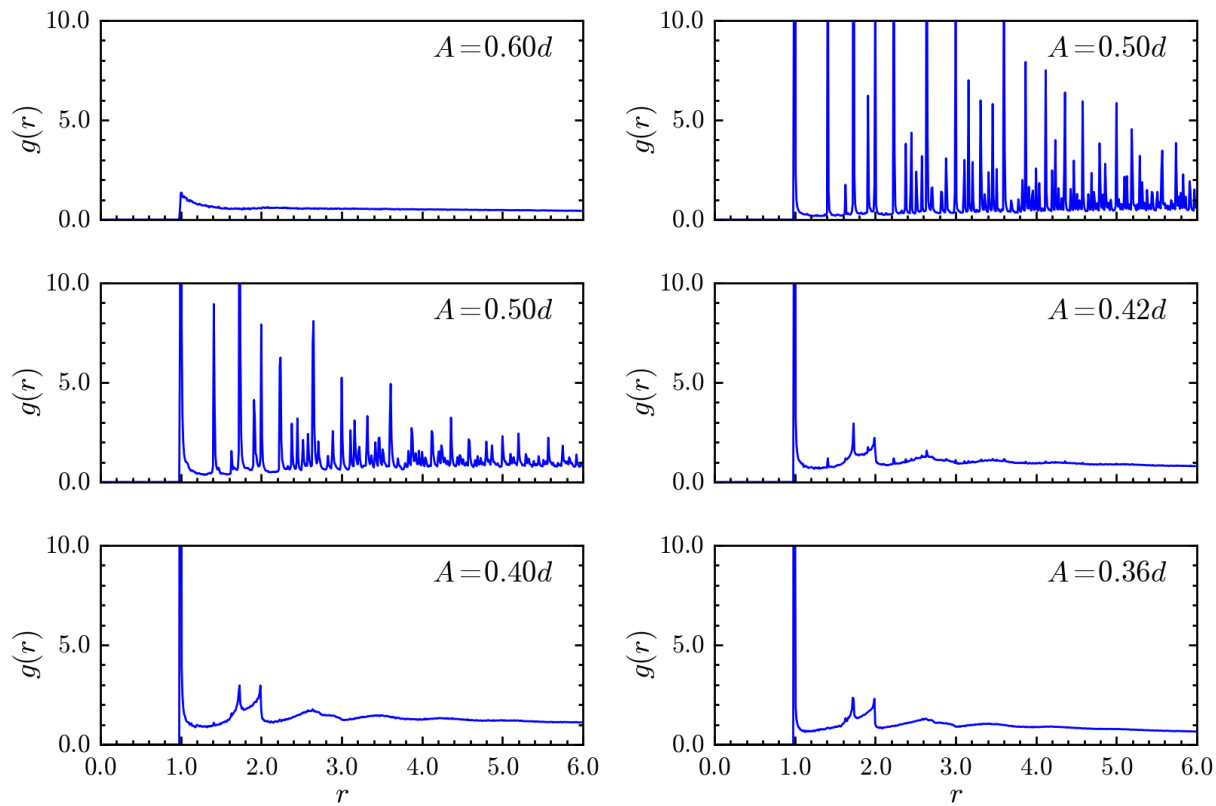

(a) $\bar{\phi}=0.16$

(b) $\bar{\phi}=0.20$
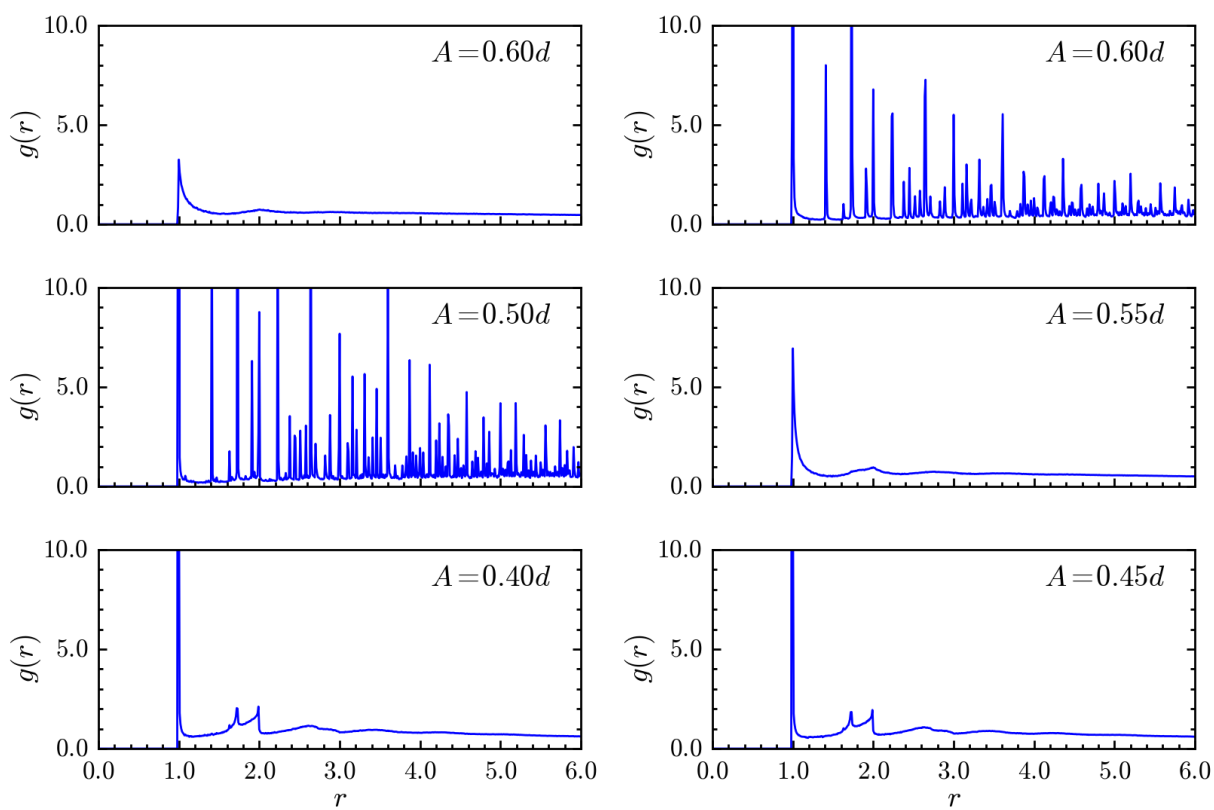

(c) $\bar{\phi}=0.23$

(d) $\bar{\phi}=0.26$

Figure 4.5: The RDF for systems with selected $A$ and $\bar{\phi}$. 
For $\bar{\phi}=0.26$ (shown in Fig. 4.5d), crystallization happens at $A=0.60 d$. Below the coexistence region, the $g(r)$ plot shows the typical structure for random close packing. As the driving amplitude increasing, the cluster will start to melt to liquid as at $A=0.55 d$ before the system starts to crystallize at $A=0.60 d$.

To sum up, below the coexistence regime, the system shows random close packing structure inside the cluster too. Above the coexistence regime, the system are homogeneous but go slowly from gas to liquid state as the averaged filling fraction increases. With higher $\bar{\phi}$ for example 0.26 , the system will go first from clustered state to homogeneous liquid, before reach the coexistence regime.

From the radial distribution function, we know that the structure of coexistence part with $\phi_{p_{\max }}$ around 0.64 is RCP. It is, however, difficult to distinguish this regime from the clustered state based only on knowledge of $g(r)$. Besides, it is hard to obtain the exact structure of the crystalline solid. To make progress, we will go to more specialized order parameters: the coordination number $Z$ and the $q_{6}$ number.

\subsection{3 $q_{6}$ and coordination number $Z$}

To detect the detailed structure of the solid plug, we calculate the local $q_{6}$ number and coordination number $Z$ for each particle, and then compute the probability distribution of the local $q_{6}$ and $Z$ for the whole system. These order parameters are shown in Fig. 4.6 and Fig. 4.7 in the space of driving amplitude $A$ and $q_{6}$ (or $Z$ ) for selected filling fractions. These plots not only clearly show the boundaries between different phases, but also show that there are fcc and hcp crystals in the solid part of PC-G state.

For $\bar{\phi}=0.14,0.16$ and 0.20 , there are two sharp peaks at about $q_{6}=0.485$ and 0.575 when $A>0.40 d$ in the $q_{6}$ histogram plot (Fig. 4.6b. Fig. 4.6c and Fig. 4.6d), together with another peak at $q_{6}=0.0$. Correspondingly, the coordination number $Z$ is about 12 in this region (Fig. 4.7b Fig. 4.7c and Fig. 4.7d) and together with another peak at $Z \leq 2$. These plots show evidence of coexistence between fcc, hcp structure and gas state. The value of $A$ where the two sharp $q_{6}$ peaks end and the $Z$ drops down below 6 marks the boundary between the coexistence regime and the homogeneous state.

In Fig. $4.6 \mathrm{~d}$ for $\bar{\phi}=0.20$, the bottom peak at $q 6=0.0$ for gas starts from $A=0.39 d$ which is the boundary between the clustered state and coexistence state. Meanwhile, from the none zero peak we can see the difference between the clustered state and the RCP part 
in the coexistent state. In the clustered state, the peak distribution is wider, from 0.30 to 0.70 . This difference cannot be seen from the $Z$ distribution (see Fig. $4.7 \mathrm{~d}$ ).

In the $q_{6}$ histogram plot for $\bar{\phi}=0.08$ (Fig. 4.6a), there is one peak at the bottom for all the $A$ shown. The other peak range from 0.40 to 0.60 will disappear as $A$ increases. In the $Z$ histogram plot (Fig. 4.7a), the first peak lies below 1. The second one lies from 7 to 10 and it will disappear too as $A$ increases. It means the system goes directly from RCP-G state to the homogeneous gas.

For $\bar{\phi}=0.14$ and 0.16 , from both the $q_{6}$ and $Z$ plots in Fig. $4.6 \mathrm{~b}$. Fig. $4.6 \mathrm{c}$ Fig. $4.7 \mathrm{~b}$ and Fig. $4.7 \mathrm{c}$ we can see that the system first goes from the RCP-G state to PC-G state, then it enters the homogeneous state. Furthermore, we can see from Fig. $4.7 \mathrm{~b}$ and Fig. $4.7 \mathrm{c}$ that, the coordination number $Z$ distribution is slightly wider for $\bar{\phi}=0.16$ than it is for $\bar{\phi}=0.14$. That give the information that the system is slowly going from the homogeneous gas to the homogeneous liquid as the averaged filling fraction increases.

For higher filling fraction $\phi=0.23$, where the "spinodal" curve is above the boundary line between RCP-G state and the PC-G state, the system goes directly from the clustered state to the PC-G state(see fig 4.6e).

For $\phi=0.26$, there is still a homogeneous region (see Fig. 4.6f) between clustered and PC-G state, which is more like the liquid (see Fig. 4.7f with a wider distribution of $Z$. As the spinodal curve at high filling fraction lies at much higher driven amplitude, the cluster will melt to liquid first before it reaches the PC-G state.

The information we gather from $q 6$ and $Z$ matches well with that from the RDF. Furthermore, we know that the crystal structures in the coexisting regime are hcp and fcc, and the clustered state has a wider $q 6$ distribution than RCP-G state. The next question is that is RCP a typical solid? In the following part we use the connection number $\xi$ calculations to answer this question.

\subsubsection{Connection number $\xi$}

We use the connection number $\xi$, as defined in the method part, to differentiate between the gas-like, liquid-like and solid-like particles. In Fig. 4.8 we show the heat maps of the $\xi$ distribution, for comparison, with the same spots in the phase diagram as we did for $q_{6}$ and $Z$. In the PC-G state, the $\xi$ distribution shows a peak at around 12 , which is consistent with the information from $Z$ and $q_{6}$. The peak around 7 to 9 in the heat map of $Z$ for the RCP-G state and clustered state does not show again in that of $\xi$. The only 


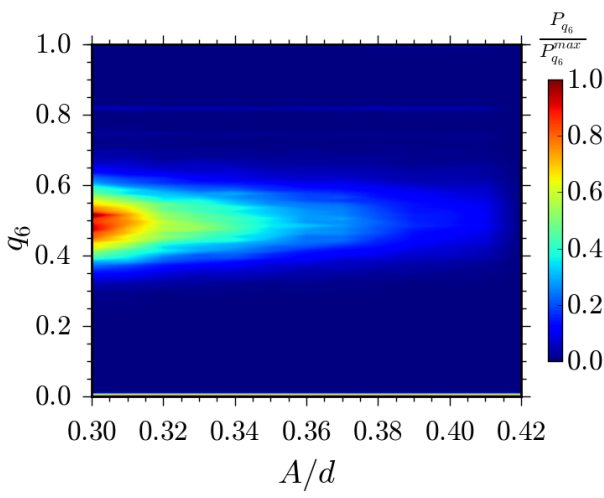

(a) $\phi=0.08$

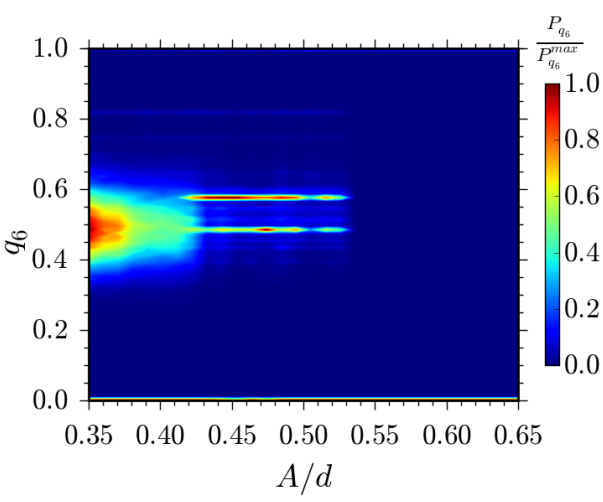

(c) $\phi=0.16$

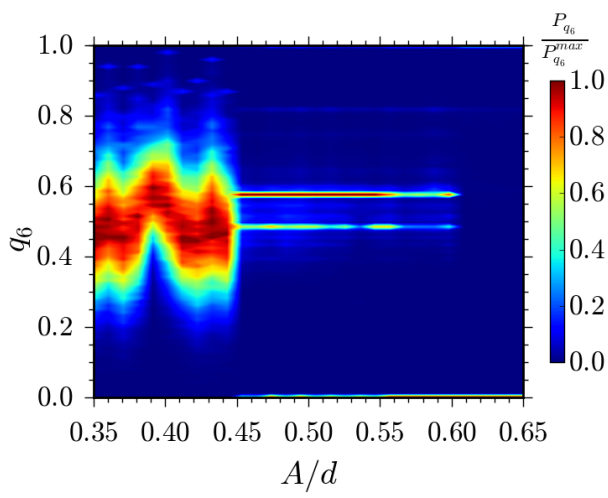

(e) $\phi=0.23$

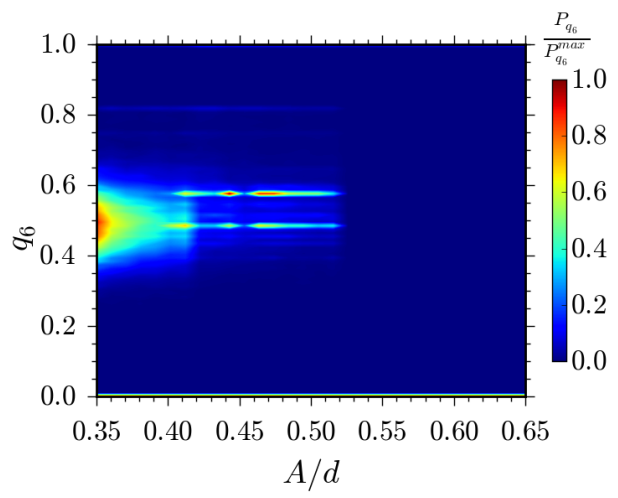

(b) $\phi=0.14$

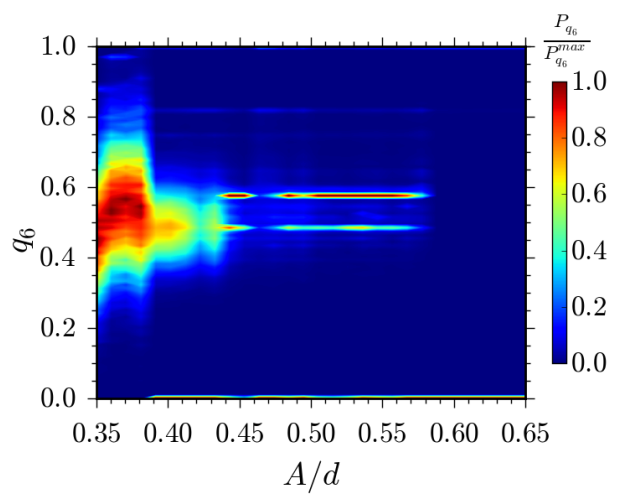

(d) $\phi=0.20$

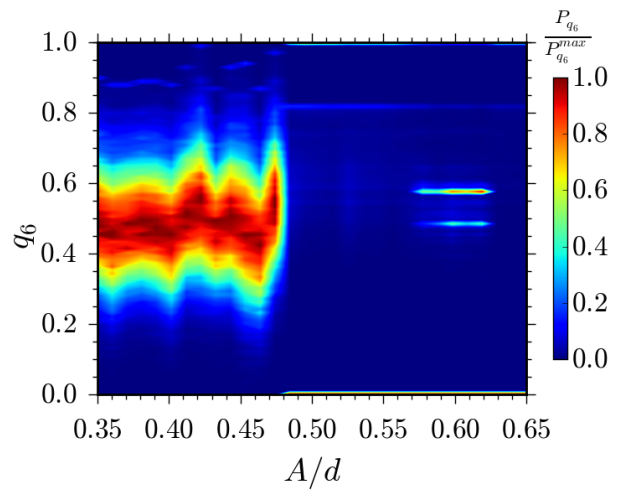

(f) $\phi=0.26$

Figure 4.6: The $q_{6}$ distribution heat maps with driving $A$ for selected $\phi$. 


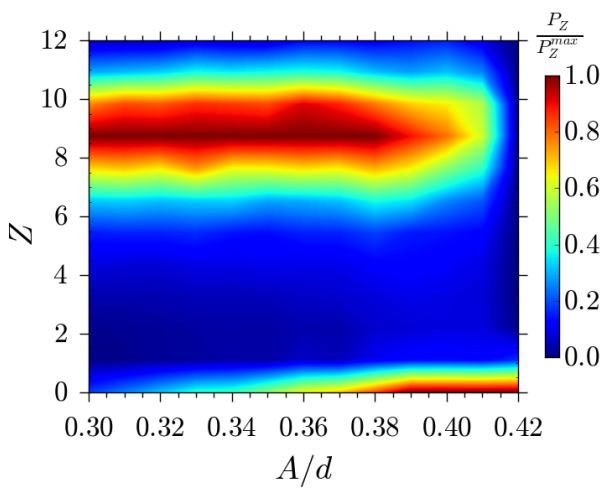

(a) $\phi=0.08$

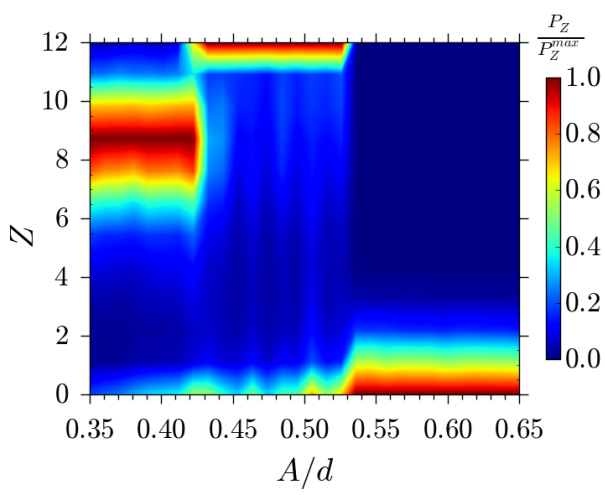

(c) $\phi=0.16$

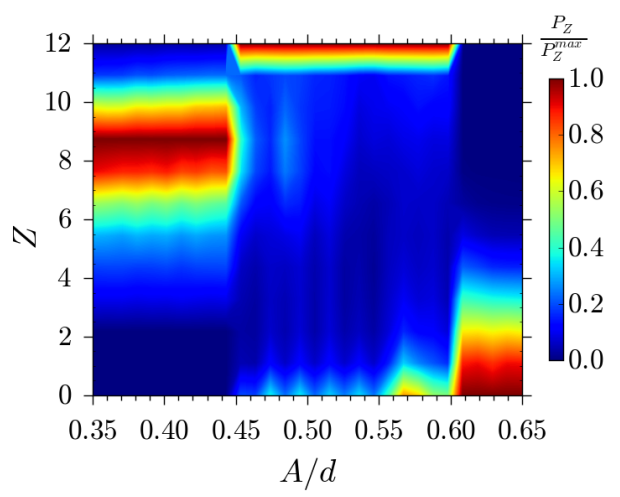

(e) $\phi=0.23$

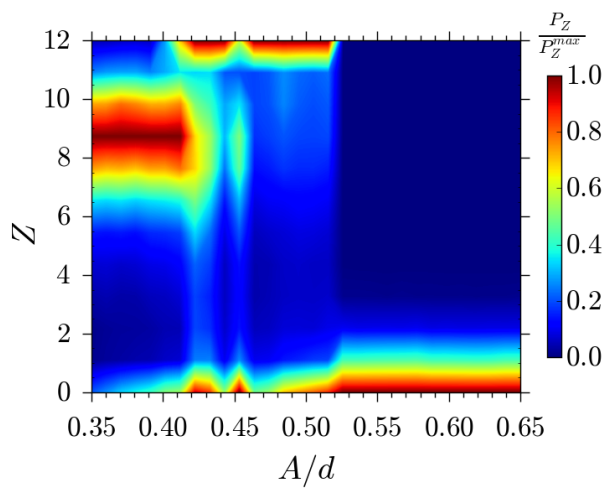

(b) $\phi=0.14$

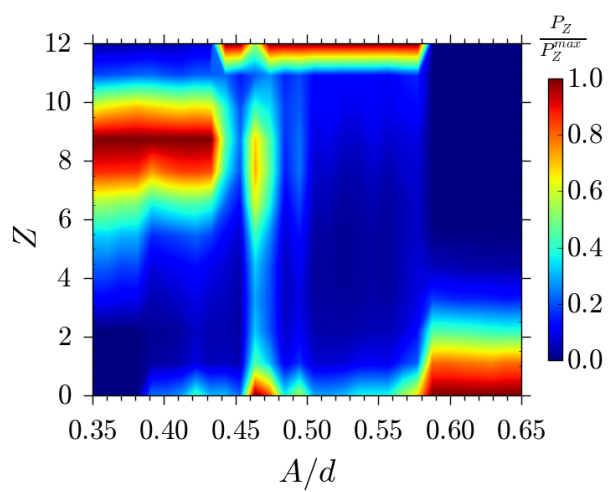

(d) $\phi=0.20$

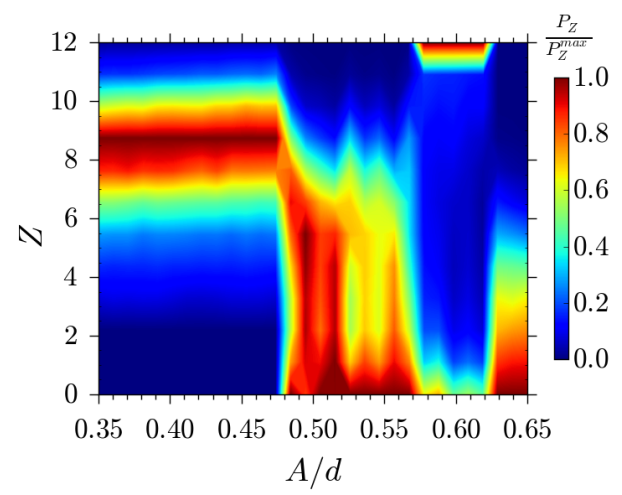

(f) $\phi=0.26$

Figure 4.7: The $Z$ distribution contour plot with driving $A$ for selected $\phi$. 


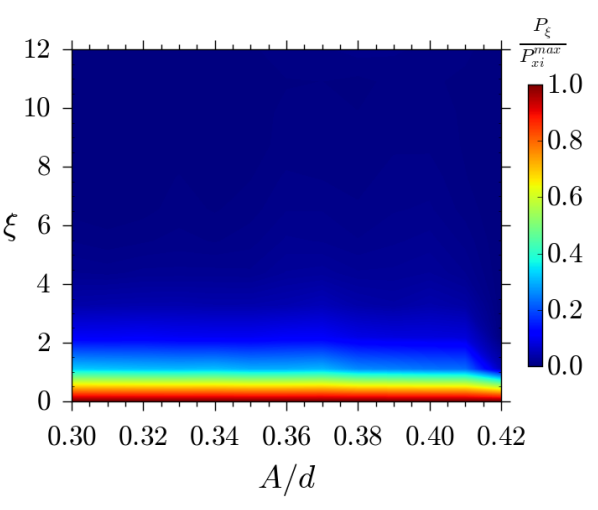

(a) $\phi=0.08$

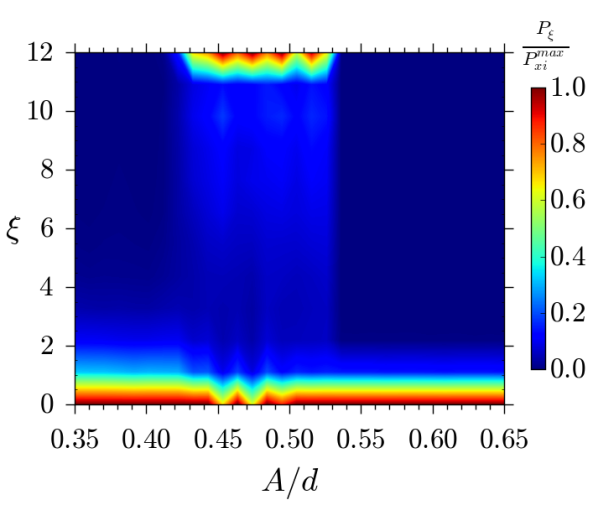

(c) $\phi=0.16$

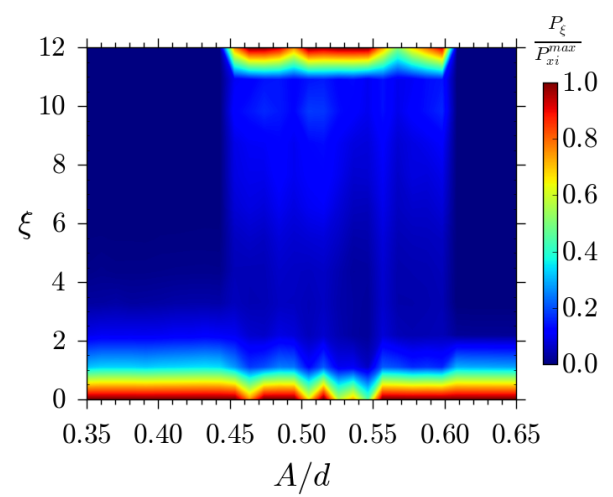

(e) $\phi=0.23$

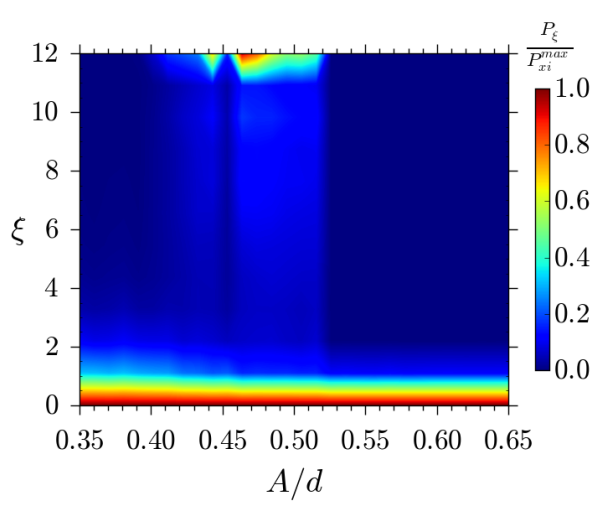

(b) $\phi=0.14$

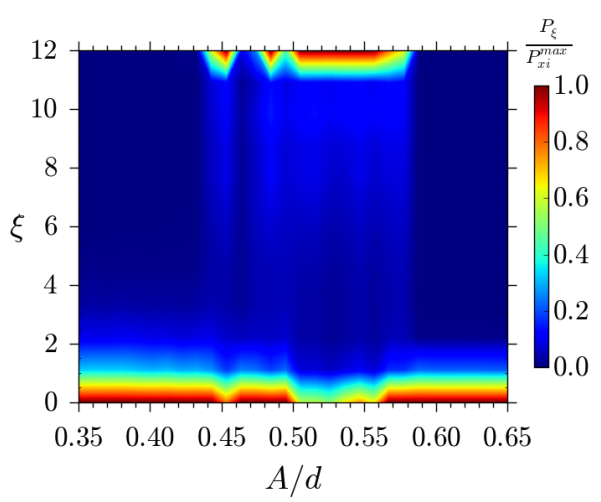

(d) $\phi=0.20$

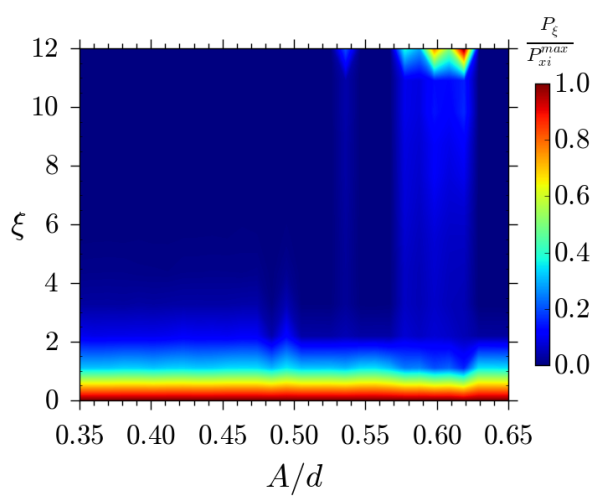

(f) $\phi=0.26$

Figure 4.8: The $\xi$ distribution heat maps with driving $A$ for selected $\phi$.

peak at this regime in the plot of $\xi$ stays below $\xi=2$ for the gas part. It means that $\mathrm{RCP}$ and clustered state are not the traditional solid we thought. 


\subsection{Conclusion}

To sum up, we use maximum weighted local filling fraction $\phi_{p_{\max }}$, radial distribution function $g(r)$, local six-fold bond orientational order parameter $q_{6}$, coordination number $Z$ and connection number $\xi$ to characterize the phases we meet in the phase space we explored and the boundaries lines between them. The local structure of the clustered state is random close packing, shown by the $g(r)$ plot. The solid part in the coexistence state exhibits either random close packing (shown by the $g(r)$ plot) or poly-crystal (hcp and fcc) structures, as shown by the $q_{6}$ and $Z$ plots. The boundary between clustered state and RCP-G state can be characterized by the $q_{6}$ distribution. Furthermore, the distribution of $Z$ shows the transfer from homogeneous gas to liquid, and the $\xi$ distribution shows that the random close packing is not a traditional solid. 



\section{Dynamics}

From the phase diagram we discussed in the previous chapter we know that there are three main regimes in the phase space we explored, and we characterized and distinguished all phases. In this chapter, we focus on how the coexisting states evolve with time. Then we will show how the crystal structure grows with time and compare it with the crystal structure discussed in the literatures about sheared granular systems. The stabilization of the crystalline structure is discussed later, where we consider the influence of driving amplitude and the gravity.

\subsection{The time evolution of coexistence phases}

To understand the process leading to phase separation in our system, we calculated the temporal evolution of the local filling fraction $\phi_{l o c}$ distribution. For a given configuration of the grains, we calculate the local filling fraction $\phi_{l o c}(i)$ for each particle $i$ based on its Voronoi volume. Then the local filling fraction distribution is the histogram of $\phi_{l o c}(i)$ of all particles in the system.

In Fig. 5.1] we show the time evolution of the local filling fraction distribution for three selected states in the phase diagram, which are representative for the RCP-G state, the PC-G state, and one spot at the boundary line between the former two phases. As a reference, the evolution of the total energy for these systems are shown alongside.

For the RCP-G state, with $A=0.36 d, \bar{\phi}=0.16$ (shown in Fig. 5.1a), two peaks in $\phi_{\text {loc }}$ appear quickly after about $t / \delta t=2 \times 10^{6}$ (100 shaking cycles), where the kinetic energy starts to exhibit oscillations. The bottom peak stays near $\phi_{l o c}=0.0$ which is for gas part and the higher one lies around $\phi_{l o c}=0.64$ which is for the random close packing.

For the PC-G state, with $A=0.50 d, \bar{\phi}=0.16$ (shown in Fig. 5.1c), similarly, two peaks appear soon after about $t / \delta t=5 \times 10^{6}$ (250 shaking cycles). However, even though the total energy has already converged to a steady state, the higher $\phi_{l o c}$ peak moves up to 

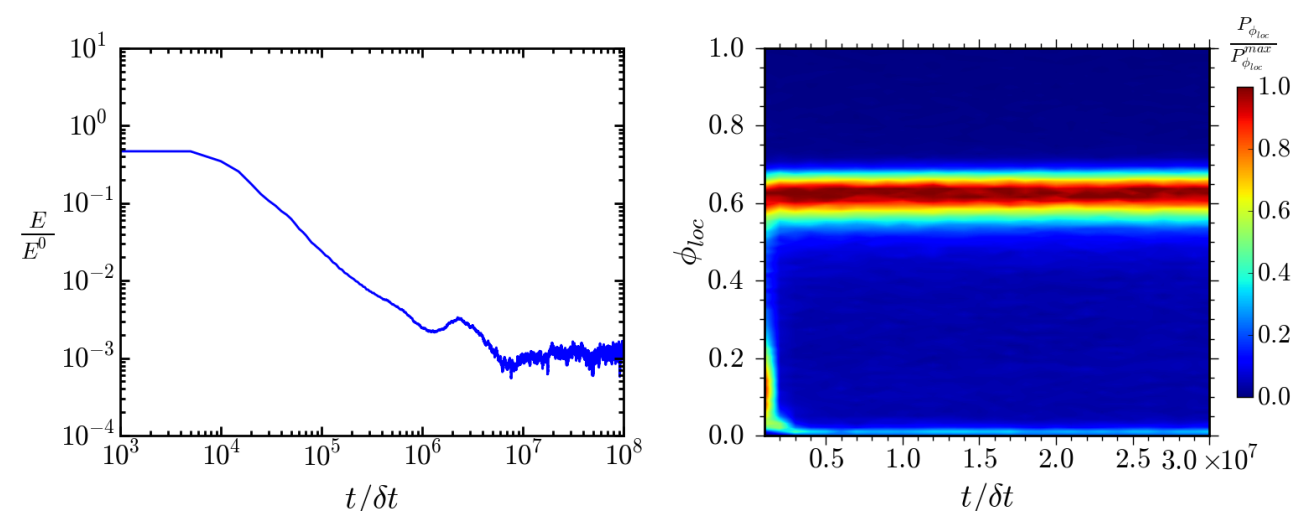

(a) $A=0.36 d$
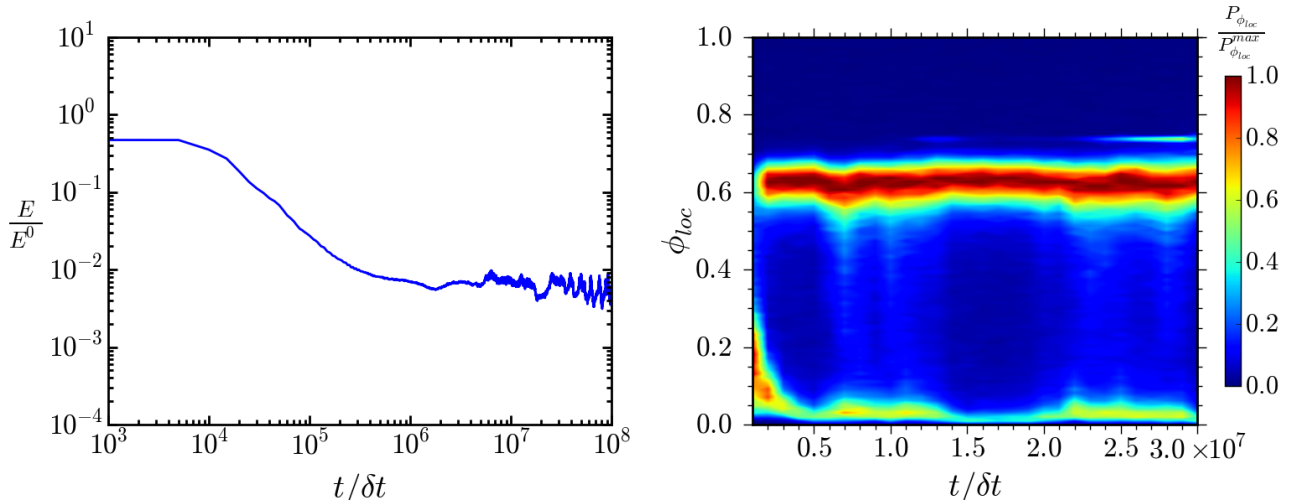

(b) $A=0.42 d$
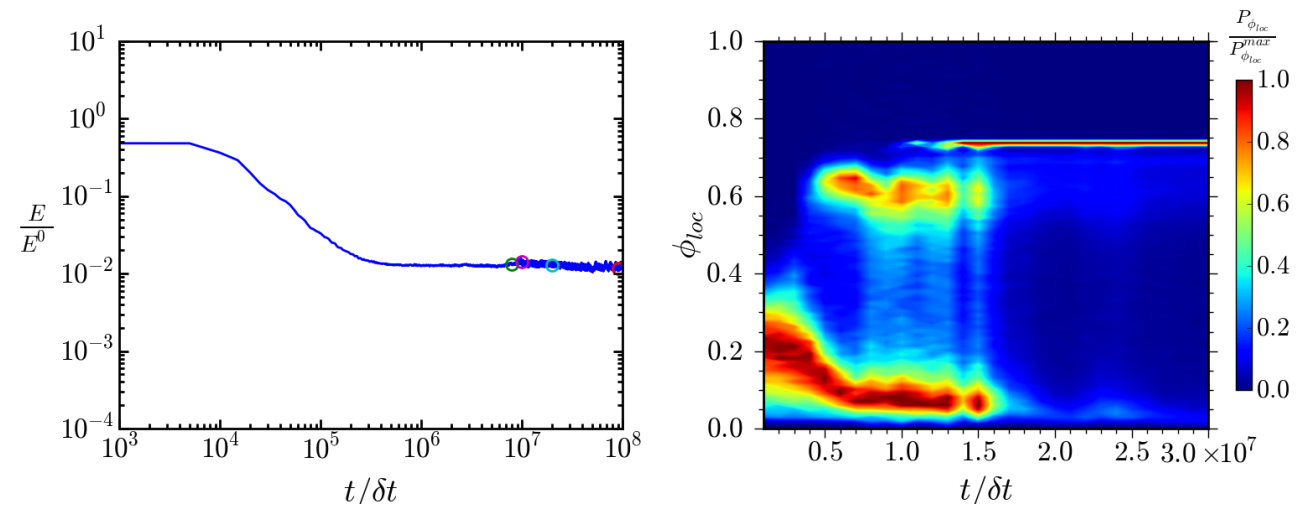

(c) $A=0.50 d$

Figure 5.1: The temporal evolution of the energy and local filling fraction $\phi_{l o c}$ distribution for systems with fixed $\bar{\phi}=0.16$ and selected $A$. 
above $\phi=0.70$ and be narrowed around the $t / \delta t=10^{7}$ time step (1000 shaking cycles). At that point, the energy starts to slightly vibrate and the system starts to crystallize.

Near the boundary line between RCP-G state and PC-G state, the third peak above $\phi_{l o c}=0.70$ shows by chance but the most weighted peak still stays at around $\phi_{l o c}=0.64$ (Fig. 5.1b). We choose one snapshot at $t / \delta=3 \times 10^{7}$ and do cluster analysis for it. Only 13 small nuclei are found and each of them includes no more than 60 particles. We will discuss their features below.

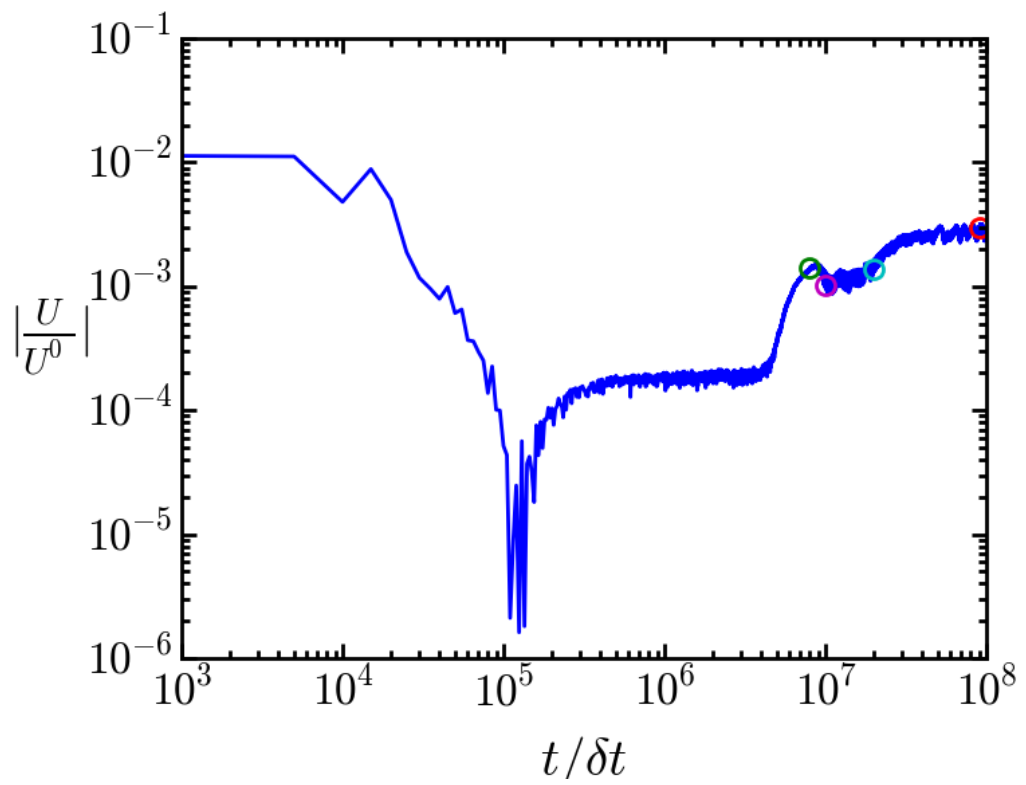

Figure 5.2: The absolute value of potential energy scaled by the initial value for system with $A=0.50 d$ and $\bar{\phi}=0.16$.

\subsection{Crystal structure growth}

In the left part of Fig. $5.1 \mathrm{c}$ the total energy varying with time is shown for $A=0.50 d, \bar{\phi}=$ 0.16 , which is at the PC-G coexistence regime. Four points in the plot are marked with green, magenta, cyan and red circles, respectively for $t / \delta t=8 \times 10^{6}, 1 \times 10^{7}, 2 \times 10^{7}$ and $9 \times 10^{7}$. The snapshots for these four time steps are shown in Fig. 5.3 where only the solid-like particles (with connection number $\xi=12$ ) are shown. Following the $q_{6}$ color map, the yellow particles have fcc local structure and the green ones have hcp structure. 
The total energy converges to a steady state through a sequence of two steps. The first one is between $t / \delta t=10^{6}$ and $10^{7}$ and the other one is after $t / \delta t=10^{7}$ where the crystallization mainly takes place. However, as shown in Fig. 5.2 the potential energy does not converge at the same regime. From the snapshots shown in Fig. 5.3a only few particles crystallized at $t / \delta t=8 \times 10^{6}$. More crystallized particles build up to form clusters at $t / \delta t=10^{7}$ (see Fig. 5.3b). Until $t / \delta t=2 \times 10^{7}$ (see Fig. 5.3c), 8 clusters have appeared and the largest one consists of 1259 particles. At $t / \delta t=9 \times 10^{7}$ (see Fig. 5.3d, close to the final state we simulated, five clusters aggregate into a big strip, and the biggest one includes up to 2736 particles. From the snapshots of the system we can see that the crystalline structures observed in our system do not have a preferential orientation.

(a) $t / \delta t=8 \times 10^{6}$

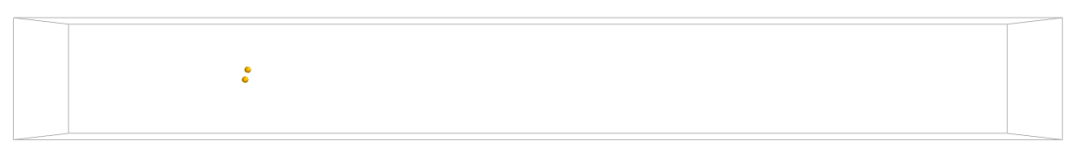

(b) $t / \delta t=1 \times 10^{7}$

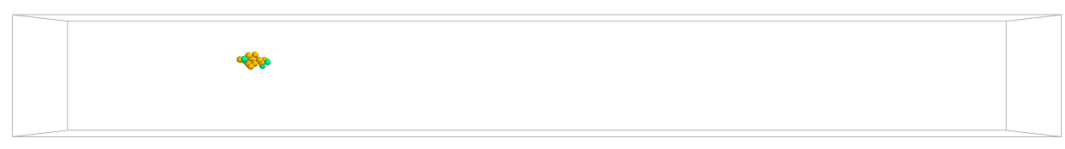

(c) $t / \delta=2 \times 10^{7}$

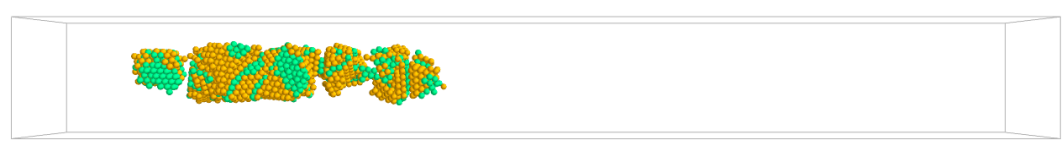

(d) $t / \delta=9 \times 10^{7}$

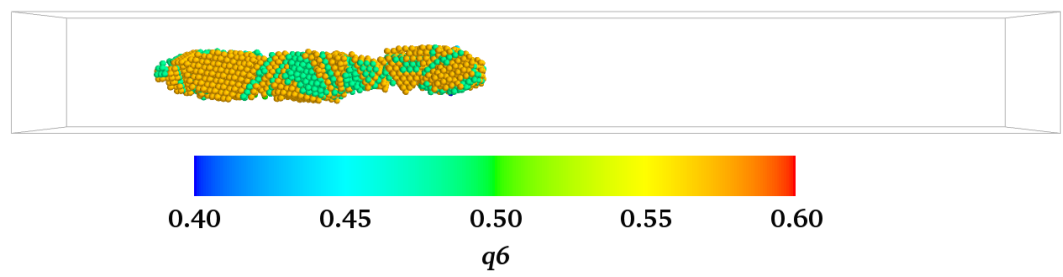

Figure 5.3: Snapshots for the selected time steps in Fig. $5.1 \mathrm{c}$ Only particles with $\xi=12$ are shown in the snapshots and the particle colors are scaled with the local $q_{6}$ numbers.

Crystallization in granular systems have been reported in the literature recently, however, 
most of them happened in sheared granular packings. In the work by A. Panaitescu et al.(93), a polycrystalline phase with domains of fcc and hcp order is observed after hundreds of thousands of shear cycles. Unlike our results, the crystal structure in their system prefer to lie parallel to the walls. F. Rietz et al.(94) studied nucleation in sheared granular system within a rough inner surface container to avoid artificial nucleation. The crystalline clusters observed in those systems do not have a preferential orientation, however, the largest cluster they measured in their experiments only includes up to 100 spheres.

\subsection{The stability of the crystal structure}

Crystallizing in our system happens with proper driving amplitudes and zero gravity. One inescapable question is whether the crystal structure generated in our system is stable or not under moderate perturbations. To answer this question, we checked the stability of the crystal structure in two cases. The first case is that we continue to drive a steady state $\mathrm{PC}-\mathrm{G}$ state system with a driving amplitude $A$ lying in the random close packing regime, and vise versa. The other one is that we slowly switch on the gravity to see if the crystallizing still happens.

\subsubsection{Irreversibility}

To check if the boundary between RCP-G state and PC-G state are reversible or not, we run the following set of simulations. We start with a system with $\bar{\phi}=0.16, A=0.50 d$, and a second one with with $\bar{\phi}=0.16, A=0.35 d$. These systems are initialized with a random, homogeneous distribution of particles' positions and brought to their respective steady states. Two additional systems are also simulated using the final particles' configurations from the above systems, but now the driving amplitudes are swapped around. These other two systems are also brought to their steady states. The steady-state pair correlation functions for all four of them are shown in Fig. 5.4

Starting from a homogeneous initial state, the system driven with $A=0.35 d$ reaches a RCP-G state (see Fig. 5.4b after $10^{8}$ time steps runs, and the system driven with $A=0.50 d$ shows signals of crystallizing (Fig. 5.4a) after the same amount of simulation time. This is consistent with the expectation based on the phase diagram in Chap. 3. We then extend the simulation at $A=0.50 d$ with $10^{7}$ additional time steps but now at the 
lower amplitude $A=0.35 \mathrm{~d}$. The pair correlation function of the final state we obtain is shown in Fig. $5.4 \mathrm{~d}$. We can see that the crystal structure still survives when we suddenly decrease the driving amplitude. However, when the system previously equilibrated at $A=0.35 d$ (in a random close packing state) is now driven for an additional $10^{7}$ time steps with $A=0.50 d$, crystal structure will arise (see Fig. $5.4 \mathrm{c}$.)

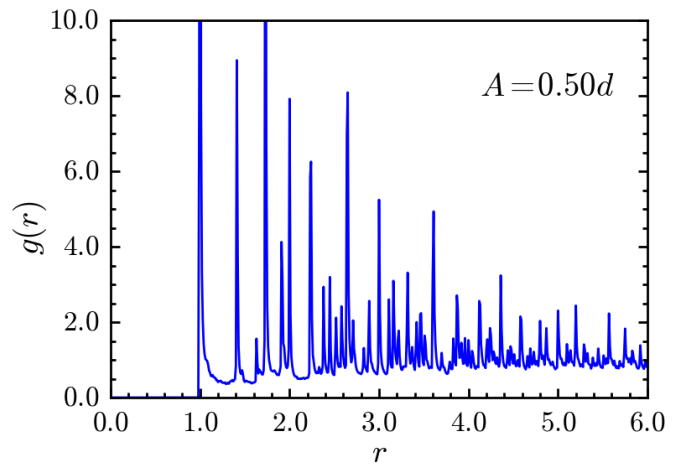

(a) random distribution initial state

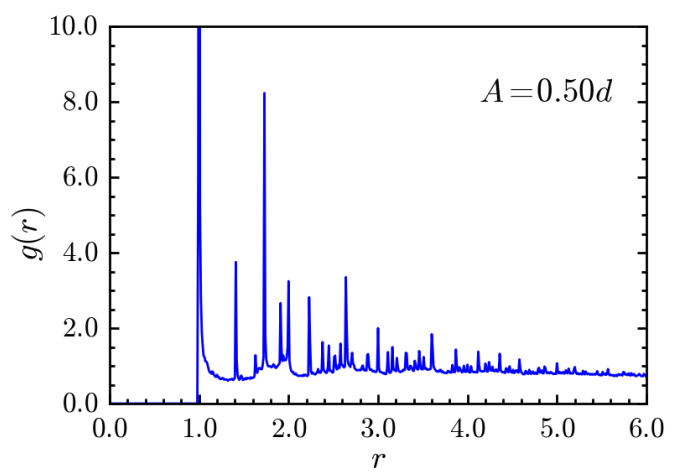

(c) initialized by steady state of $A=0.35 d$

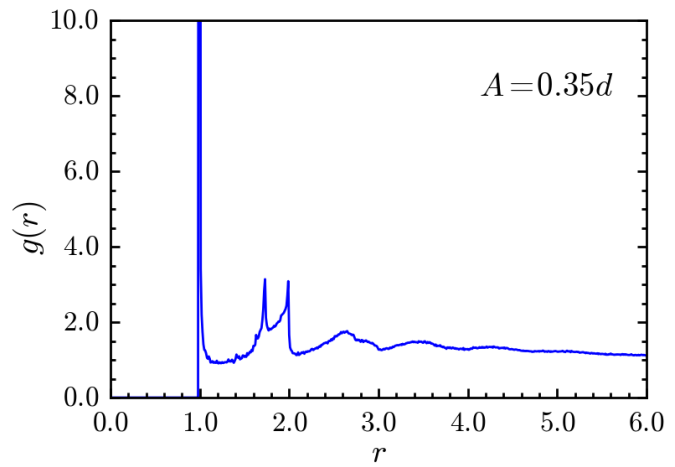

(b) random distribution initial state

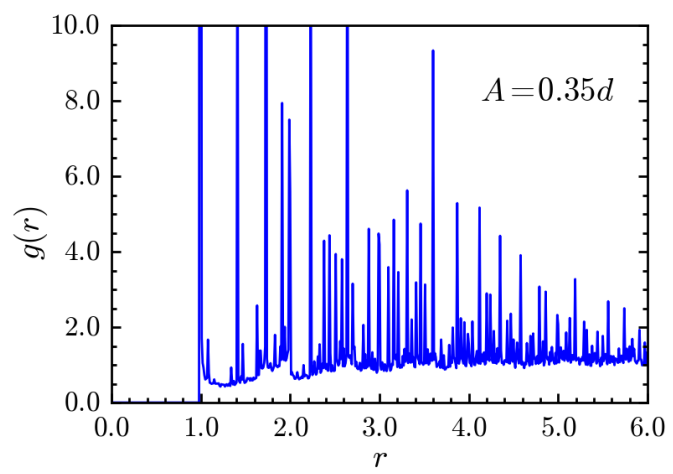

(d) initialized by steady state of $A=0.50 d$

Figure 5.4: The poly-crystal structure and random close packing stability check by swapping the driving amplitude for $\bar{\phi}=0.16$. We show the RDF to characterize the structure of the system. See the main text for details about the simulation protocol.

We can then conclude the following: the crystal structure appearing in our phase diagram is irreversible and will not be destroyed by decreasing the driving amplitude. However, the random close packing state could crystallize when we increase the driving amplitude to PC-G state regime. 


\subsubsection{Influence of gravity}

All the results shown until now are from simulations with zero gravity. This is an extremely artificial situation; hence we perform additional simulations with gravity too, to detect how the gravity will influence our phase diagram. The added gravity is along the $z$ direction of the simulation domain. Four different gravity accelerations are selected and they are respective $g=0.0001 \mathrm{~m} / \mathrm{s}^{2}, 0.01 \mathrm{~m} / \mathrm{s}^{2}$ and $0.1 \mathrm{~m} / \mathrm{s}^{2}$. The $q_{6}$ and coordination number $Z$ distributions in the space of amplitude and $q_{6}$ (or $Z$ ) are shown in Fig. 5.5 The main conclusion from an inspection of the results is that our solid phases survive with weak gravity.

With $g=0.0001 \mathrm{~m} / \mathrm{s}^{2}$ (Fig. 5.5a), the histogram of $q 6$ and $Z$ do not show any significant difference from the zero-gravity results. The $g=0.01 \mathrm{~m} / \mathrm{s}^{2}$ gravity (Fig. $5.5 \mathrm{~b}$ ) will completely destroy the phase separation and from the histogram of coordination number $Z$ we could see the system will stay at homogeneous gas state at our simulation regime. With gravity up to $g=0.1 \mathrm{~m} / \mathrm{s}^{2}$, stronger driven will fluidized the granular particles and all the particles will collapse to the bottom wall(see Fig. 5.6 at relatively weaker amplitude.

\subsection{Conclusion}

To sum up, the system starts to crystallize when the driving amplitude is above a threshold value of the driving amplitude: the boundary line between the RCP-G state and the PC$\mathrm{G}$ state. The crystallization happens at the time when the potential energy starts to converge to a steady state, which is much later then the time when total energy starts to converge. This was made possible only because of the considerable long simulation times we were able to carry out. The crystallization is not introduced by the confinement of walls, and it starts from within the solid plug rather than from the boundaries between particles and the walls. The crystallizing process is irreversible. With gravity, the phase separation to PC-G state can arise only when gravity is very weak. 

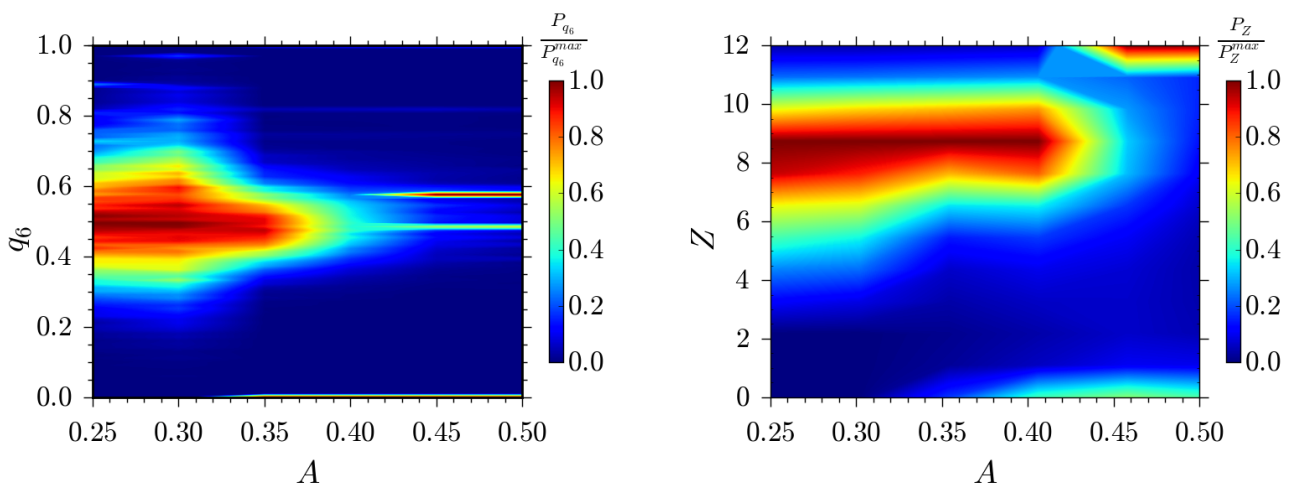

(a) $g=0.0001$
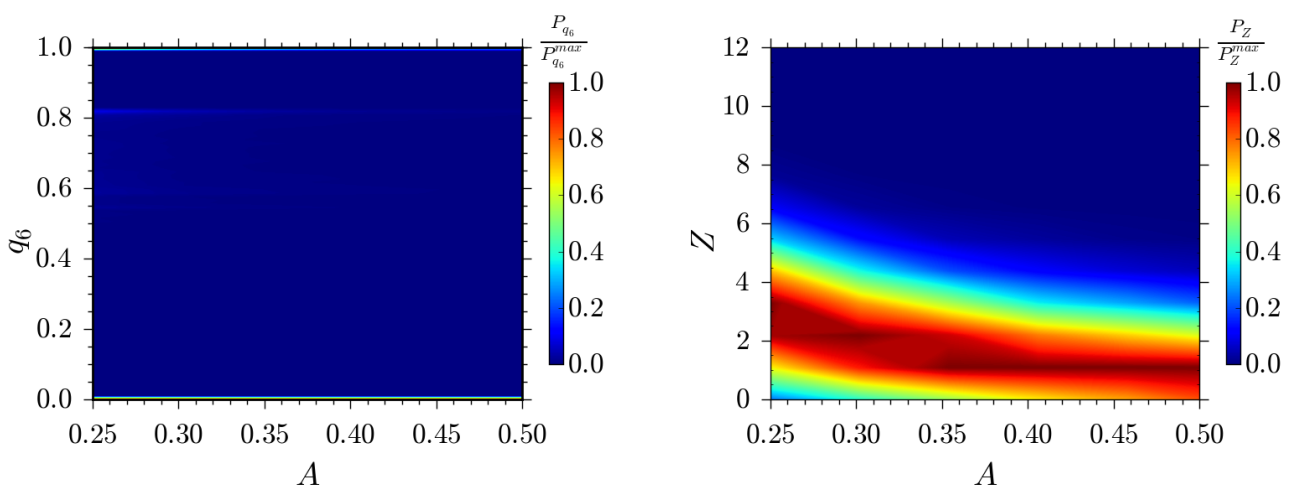

(b) $g=0.01$
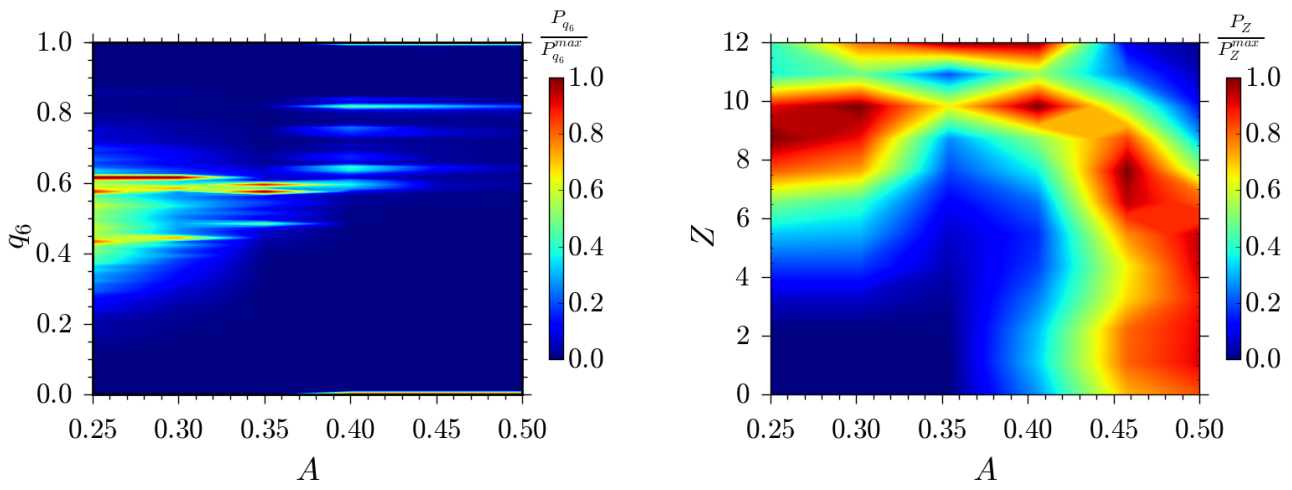

(c) $g=0.1$

Figure 5.5: Heat maps for $q_{6}$ and $Z$ for systems at fixed $\bar{\phi}=0.16$ and with varying strength of gravity. 


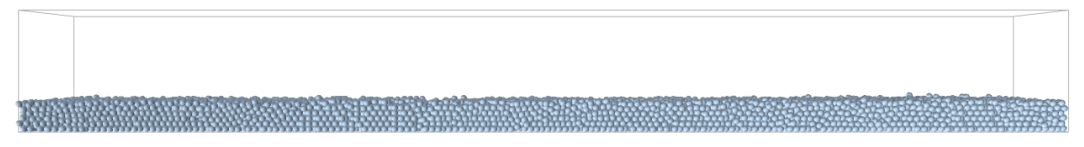

Figure 5.6: One steady state snapshot for system with $A=0.30 d, \bar{\phi}=0.16$ and $g=0.1$ $\mathrm{m} / \mathrm{s}^{2}$. 



\section{Coexistence regions}

The coexistence states we observed in the phase diagram we explored only exist in the granular system with van der Waals interactions, especially the PC-G state. In a granular system without van der Waals interactions, the denser part in the coexistence state can not reach the filling fraction of random close packing even with a coefficient of restitution as low as $\epsilon=0.6$. In the van der Waals gas, liquid-gas coexistence can be seen when the equilibrium temperature is low enough (95). Therefore, the phase separation occurring in our system is caused by the combined effect of van der Waals interaction and dissipation in granular system.

In this chapter, we study how the dissipation and the strength of van der Waals interaction influence the boundary line position between RCP-G state and PC-G state.

\subsection{Influences of dissipation}

In Fig. 6.1 we show the phase diagram in the $\epsilon$ and $A$ space at a fixed filling fraction $\phi=0.16$. When $\epsilon$ decreases, i.e. increasing the dissipation, the interval of driving amplitudes where phase separation occurs will expand and shift to a stronger driving regime. Two kinds of coexistence states appear in the separation regime. The RCP-G state will change into PC-G state as the driving amplitude increases. The crystalline solid block in the coexistence state may melt into liquid with small enough $\epsilon$ and large driving amplitude.

To determine the boundary line between the RCP-G state and the PC-G state in the space of $\epsilon$ and $A$, we carried out more simulations with different filling fractions. The results are shown in Fig. 6.2 We found that the boundary lines from different filling fraction converge to one line. The result can be fitted by the equation $c_{1} A^{c_{2}}=1-\epsilon^{2}$ (plotted together with the simulations in Fig. 6.2). The fitting line converges to $A=0$ 


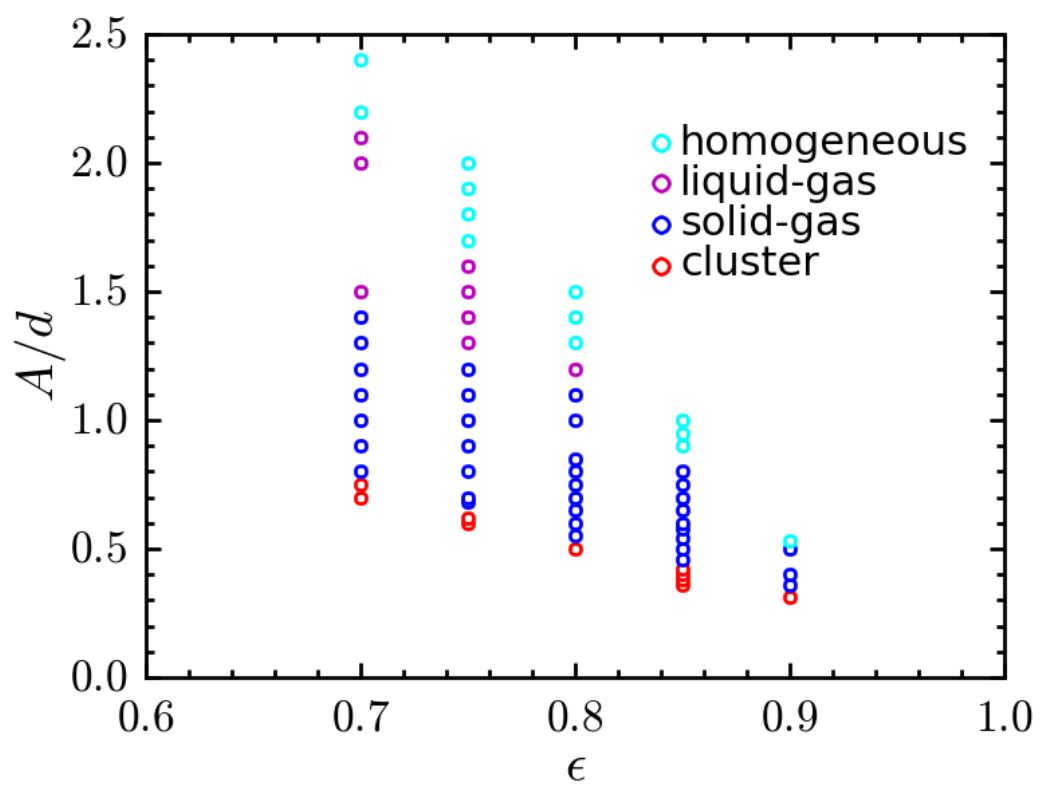

Figure 6.1: The phase diagram in the $\epsilon-A$ space.

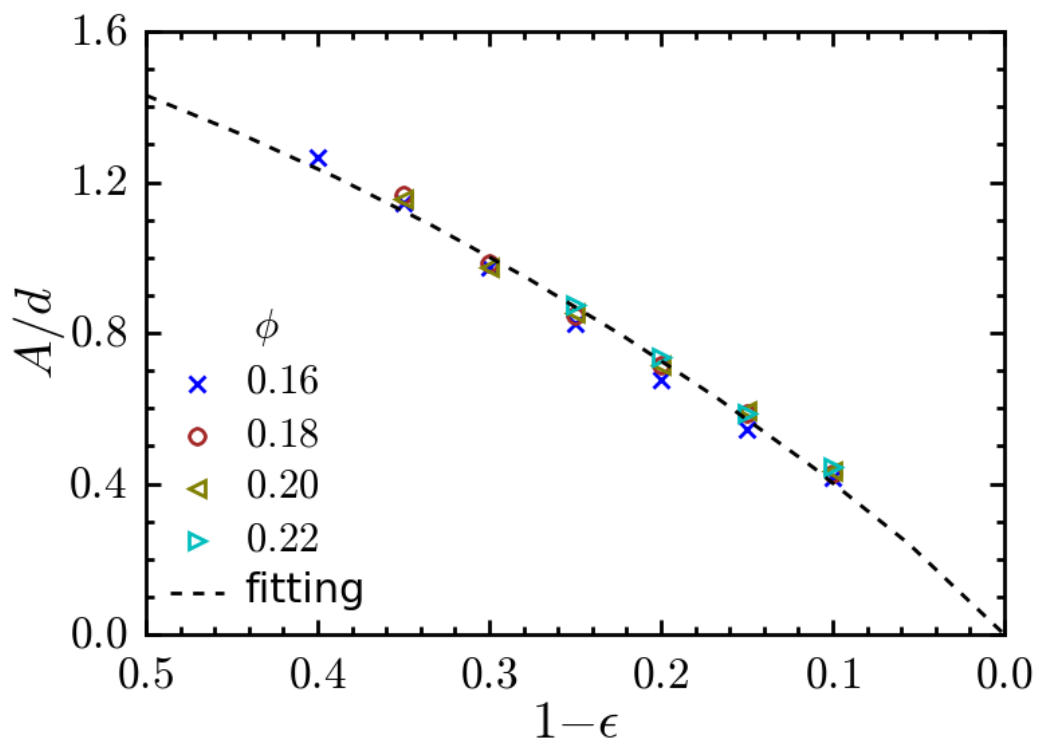

Figure 6.2: The boundary line of RCP-G state and PC-G state in the space of $\epsilon-A$. 
as $\epsilon \rightarrow 1$, which is consistent with the argument that there is no PC-G state appearing in the equilibrium van der Waals gas system.

As the phase separation occurs in a region where the driving is quite gentle, at the very beginning of a simulation the injected energy is not enough to balance the dissipation by collisions. The system effectively experiences a moderate dissipative cooling process before phase separation. We find that the boundary between RCP-G state and PC-G state is decided by $\mathcal{E}$, which is defined as the ratio between the energy injection rate $e_{i n j}$ and the energy dissipation rate $e_{d i s s}$.

Recently, A. Sack et al. (96) investigated the energy dissipation rate for a sinusoidally vibrated granular system in zero gravity environment. They identified that there are two different dissipation modes in a driving granular system, depending on the driving amplitude.

One threshold $A_{0}$ is defined as

$$
A_{0}=\frac{L_{g}}{\pi}
$$

where $L_{g}$, named as clearance, is the width of the gap between the solid walls in the driving direction and the thickness of the packed layer of particles in the box. For intense driving $A>A_{0}$, the center of mass of the granulate moves synchronously with the driven container. This regime is called collect and collide mode. While for weak driving $A<A_{0}$, the granular particle exhibit gas-like behavior. The dissipation mechanisms in the two regimes are different, leading to different dissipation rates dependence on the exciting amplitude.

The filling fractions in our explored phase diagram reaches up to 0.30. Compared with the simulation box size, the $L_{g}$ of our system is roughly three times the particle diameter. The driving amplitudes we used here are always much smaller than $L_{g}$, so our system is in the weak driving mode. During the cooling process, the system is in a gas-like state.

If we assume the system with homogeneous density, the walls always feel a constant pressure $P$ from the confined granular gas. The pressure $P$ is proportional to the particlewall collision rate $f^{p w}$. The energy injection over one period of oscillation is the work walls have done to the granular gas. So, the energy injection rate can be written as

$$
e_{i n j} \propto f^{p w} A
$$

where $e_{i n j}$ is assumed independent of the driving frequency. This is consistent with the experimental results by A. Sack et al. (96) . All their measurements were performed 
in the steady state, so the energy dissipation rate is equivalent to the energy injection rate.

Again as the system remains in the gas state, based on the kinetic theory, the dissipation rate for a homogeneous granular gas is

$$
e_{d i s s}=f^{p p}\left(1-\epsilon^{2}\right)
$$

where $f^{p p}$ is the particle-particle collision frequency. The relationship between $f^{p p}$ and $f^{p w}$ is

$$
f^{p w}=C f^{p p}
$$

where $C$ is a constant depending on the system length in the driving direction.

So the ratio $\mathcal{E}$ can be written as

$$
\mathcal{E} \propto \frac{A}{1-\epsilon^{2}}
$$

The fit to the data in Fig. 6.2 is in good agreement with $\mathcal{E}=$ const, which supports the picture that a characteristic value of $\mathcal{E}$ is required to turn the RCP-G state into a PC-G state. This should be physically linked to the nucleation barrier of the crystalline states. However, this kind of analysis is prohibitive in our granular system, so we cannot directly probe the nucleation barrier. For increasing dissipation $(1-\epsilon)$, stronger driving amplitudes are then necessary to overcome the dissipative 'trap' and lead to reorganization into a crystalline structure. Interestingly, we note an analogy to the quenching of an equilibrium liquid: very rapid cooling may avoid the crystal nucleation and result in a metastable supercooled liquid. Similarly, in our granular system, a rapid cooling may avoid ordered structures and result in a metastable RCP state.

\subsection{The influence by the van der Waals interaction strength}

According to our results, the solid-gas phase separation shown above does not exist in a vibrated granular system without van der Waals interaction. Hence, the van der Waals interaction is another prerequisite for the present phase separation. The attractive interactions trap the particles with energy below a material-dependent threshold within 


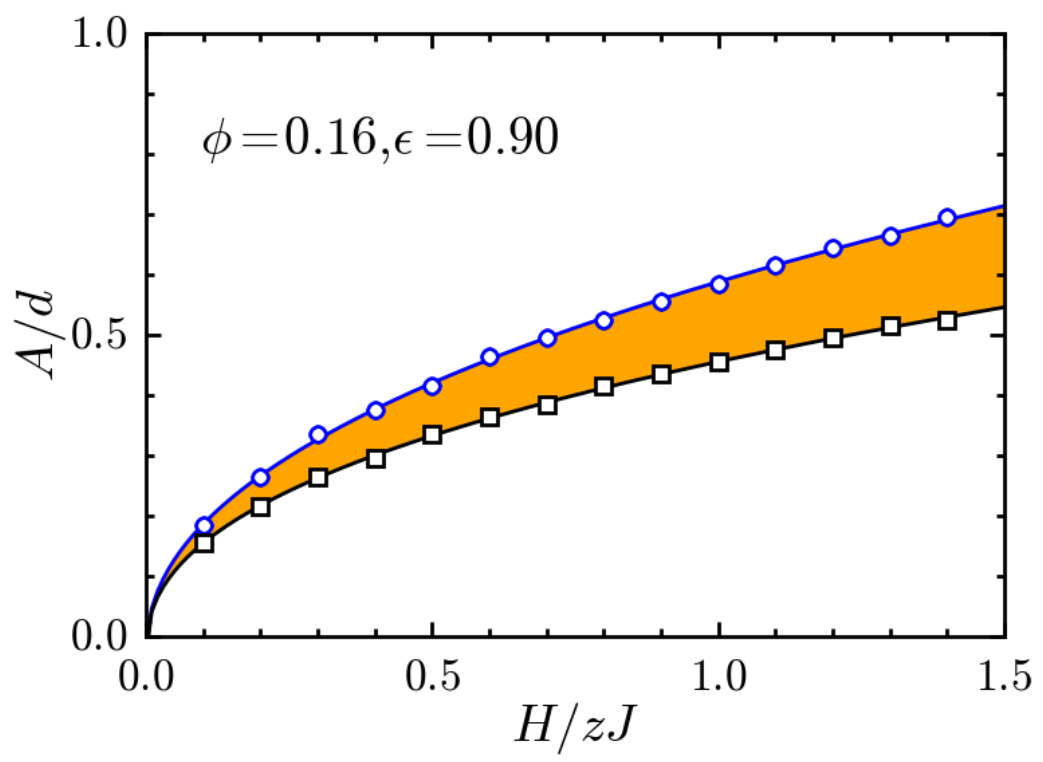

Figure 6.3: The phases boundaries vary with the Hamaker constant for a system with $\bar{\phi}=0.16$ and $\epsilon=0.9$. The blue line is the boundary between the homogeneous state and crystalline-gas coexistence state. The black line is the boundary between the PC-G and RCP-G state. Circles are from simulation data and the solid lines are fits. 
potential wells. This would imply that stronger attractive forces should increase the domain of stability of the PC-G state. Figure 6.3 confirms this expectation. We show the phase boundaries in the space of Hamaker constant and driving amplitude $A$. For a given material, there will be a corresponding interval of $A$ where the system shows a PC-G state. As the Hamaker constant increases, i.e., the strength of attractive interactions increases, the PC-G state regime will expand and shift to higher driving values. 


\section{Conclusion}

\subsection{Summary}

The work reported in this thesis is devoted to study how the collective behavior of a driven granular gas changes when van der Waals interactions among the grains are introduced.

On the one hand, in the equilibrium van der Waals gas, a well-known liquid-gas coexistence state can be seen when the equilibrium temperature is low enough (95). On the other hand, in a granular system with not van der Waals forces a liquid-gas coexistence state may emerge as the system is vibrated (49). One question then arises: how would a driven granular system behave when van der Waals interactions are included? Does it still show the liquid-gas coexistence state, or new phases will appear?

To answer these questions, we numerically simulated a sinusoidally driven granular system with van der Waals interactions among the particles. The geometrical and physical characteristics of the simulations were selected in close connections to the noncohesive granular system where liquid-gas coexistence was observed (49). The granular particles are identical spheres with radius $70 \mu \mathrm{m}$, a size for which van der Waals interactions are recognized to play a role. The van der Waals interactions are described by the well-known Hamaker theory, which are normally used to describe the van der Waals interactions among macroscopic objects.

We explore the phase diagram spanned by the average filling fraction and driving amplitude. Surprisingly, a solid-like and gas coexistence regime is found in the region where the van der Waals potential energy is comparable with the granular temperature. The liquid-gas coexistence regime is still there at larger driving amplitudes, as the van der Waals potential is too weak to influence that region, and the new solid-like and gas coexistence lies below it and remains at the relatively weak driving regime in the phase diagram. That is consistent with the properties of van der Waals interaction, frequently creating condensed phases out of a gas. Furthermore, we find that the solid-like part of 
the coexistence state can be either random close packing or exhibit a poly-crystal structure depending on the driving amplitude. The boundary line between the coexistence of random close packing and gas and the coexistence of poly-crystal and gas are parallel to the filling fraction axis, which means the transition between these two coexistence states are energy-driven.

Dissipative collisions and van der Waals interactions are two essential ingredients for the separation, as the solid-like and gas phase separation happen neither in the equilibrium van der Waals gas nor in the 'dry' driven granular system. Hence, we then focus on how the restitution coefficient (used to describe the dissipation) and the Hamaker constant (used to describe the van der Waals strength) influence the coexistence region, especial the boundary line between the random close packing and gas coexistence and the polycrystal and gas coexistence. We found that the coexistence regime expands and shifts to stronger driving regimes when either the dissipation increases or the van der Waals forces among particles become stronger. And the boundary line between the two different coexistence regimes lies on the locus of constant $\mathcal{E}$. $\mathcal{E}$ is defined as the ratio between the energy injection rate $e_{i n j}$ and the energy dissipation rate $e_{d i s s}$.

As the phase separation takes place in a region where the driving is quite gentle, at the very beginning the injected energy is not enough to balance the dissipation by collision and the system effectively undergoes a granular cooling process before separation. The ratio of energy injection rate and the energy dissipation rate during the cooling process determines the final state of the denser part in the coexistence state. Analogous to the quenching of an equilibrium liquid, higher energy injection rate, i.e. slow cooling, allows the system to crystallize to fcc or hcp structure, while the rapid dissipation, i.e. rapid cooling, restrains the nucleation so that the denser part will rest on a metastable state random close packing.

Furthermore, we find the location of the poly-crystal and gas coexistence regime in the Hamaker constant and driving amplitude space. As a reference, it can be used to determine the necessary driven energy for a specific material, and vice versa, we could predict the material inside a crystallized bulk which was generated in a specific driving environment. 


\subsection{Discussion and outlook}

The work reported in this thesis considers an ideal case, in which all particles are identical in size, shape and material properties. All the interactions are described by the simplest models. The external driving is only at the boundary of the system and the driving frequency is unique. More practical condition should be considered.

\subsubsection{Polydispersity}

It is common to use slightly polydisperse spheres in simulations to avoid crystallization. If the crystal structure we have seen in our system will survive in a system consisting of polydisperse spheres, and how the polydispersity will influence the collective behavior of granular system with van der Waals interaction are important questions we need to study in future.

\subsubsection{Frequency influence}

In the work reported by K. Roeller et al. (49), it was found that the phase separation only appears at some special driven frequency regime. Their explanation is that if the resonant motion of a single particle is suppressed the collective coherent motion is destroyed and the phase separation disappears.

The phase separation we see in our system appears in the region where the van der Waals potential energy is comparable with the granular temperature. The question whether the resonance with the driving frequency is again a crucial reason for the the separation or not is also important for understanding the mechanism of separation in our system and worth further study. 



\section{Bibliography}

[1] Barabási, A.-L., Albert, R. \& Schiffer, P. The physics of sand castles: maximum angle of stability in wet and dry granular media. Physica A: Statistical Mechanics and its Applications 266, 366 (1999). URL http://dx.doi.org/10.1016/S0378-4371(98) 00618-9

[2] Lee, J. \& Herrmann, H. J. Angle of repose and angle of marginal stability: molecular dynamics of granular particles. Journal of Physics A: Mathematical and General 26, 373 (1993). URL http://stacks.iop.org/0305-4470/26/i=2/a=021.

[3] Ristow, G. H. Dynamics of granular materials in a rotating drum. Europhysics Letters 34, 263 (1996). URL http://stacks.iop.org/0295-5075/34/i=4/a=263.

[4] Schwarzer, S. Sedimentation and flow through porous media: Simulating dynamically coupled discrete and continuum phases. Physical Review E 52, 6461 (1995). URL http://dx.doi.org/10.1103/PhysRevE.52.6461.

[5] Tsuji, D., Otsuki, M. \& Katsuragi, H. Relaxation dynamics of a granular pile on a vertically vibrating plate. Physical Review Letters 120, 128001 (2018). URL http://dx.doi.org/10.1103/PhysRevLett.120.128001.

[6] Hong, D. C., Quinn, P. V. \& Luding, S. Reverse brazil nut problem: Competition between percolation and condensation. Physical Review Letters 86, 3423 (2001). URL http://dx.doi.org/10.1103/PhysRevLett.86.3423

[7] Williams, J. The segregation of particulate materials. a review. Powder Technology 15, 245 (1976). URL http://dx.doi.org/10.1016/0032-5910(76)80053-8

[8] Knight, J. B., Jaeger, H. M. \& Nagel, S. R. Vibration-induced size separation in granular media: The convection connection. Physical Review Letters 70, 3728 (1993). URL http://dx.doi .org/10.1103/PhysRevLett.70.3728 
[9] Jullien, R., Meakin, P. \& Pavlovitch, A. Three-dimensional model for particle-size segregation by shaking. Physical Review Letters 69, 640 (1992). URL http://dx. doi.org/10.1103/PhysRevLett.69.640.

[10] Rosato, A., Strandburg, K. J., Prinz, F. \& Swendsen, R. H. Why the brazil nuts are on top: Size segregation of particulate matter by shaking. Physical Review Letters 58, 1038 (1987). URL http://dx.doi.org/10.1103/PhysRevLett.58.1038.

[11] Mao-Bin, H., Qing-Song, W. \& Rui, J. Size segregation in a vibrated tilted compartmentalized granular chamber. Chinese Physics Letters 20, 1091 (2003). URL http://stacks.iop.org/0256-307X/20/i=7/a=335.

[12] Shinbrot, T. \& Muzzio, F. J. Reverse buoyancy in shaken granular beds. Physical Review Letters 81, 4365 (1998). URL http://dx.doi.org/10.1103/PhysRevLett. 81.4365.

[13] Cooke, W., Warr, S., Huntley, J. M. \& Ball, R. C. Particle size segregation in a twodimensional bed undergoing vertical vibration. Physical Review E 53, 2812 (1996). URL http://dx.doi.org/10.1103/PhysRevE.53.2812.

[14] Schnautz, T., Brito, R., Kruelle, C. A. \& Rehberg, I. A horizontal brazil-nut effect and its reverse. Physical Review Letters 95, 028001 (2005). URL http://dx.doi. org/10.1103/PhysRevLett.95.028001.

[15] Shinbrot, T., Sabuwala, T., Siu, T., Vivar Lazo, M. \& Chakraborty, P. Size sorting on the rubble-pile asteroid itokawa. Physical Review Letters 118, 111101 (2017). URL http://dx.doi.org/10.1103/PhysRevLett.118.111101.

[16] Jullien, R. \& Meakin, P. A mechanism for particle size segregation in three dimensions. Nature 344, 425 (1990). URL http://dx.doi.org/10.1038/344425a0

[17] Duran, J., Rajchenbach, J. \& Clément, E. Arching effect model for particle size segregation. Physical Review Letters 70, 2431 (1993). URL http://dx.doi.org/ 10.1103/PhysRevLett.70.2431.

[18] Bernal, J. D. A geometrical approach to the structure of liquids. Nature 183, 141 (1959). URL http://dx.doi.org/10.1038/183141a0.

[19] Gennes, P. G. D. Superconductivity Of Metals And Alloys (2nd Edition) (Westview Press, 1999). 
[20] Clauss, W. et al. Self-organized critical behaviour in the low-temperature impact ionization breakdown of p-ge. Europhysics Letters 12, 423 (1990). URL http: //stacks.iop.org/0295-5075/12/i=5/a=008

[21] Bassett, D. S., Owens, E. T., Porter, M. A., Manning, M. L. \& Daniels, K. E. Extraction of force-chain network architecture in granular materials using community detection. Soft Matter 11, 2731 (2015). URL http://dx.doi.org/10.1039/c4sm01821d.

[22] Jain, N., Khakhar, D. V., Lueptow, R. M. \& Ottino, J. M. Self-organization in granular slurries. Physical Review Letters 86, 3771 (2001). URL http://dx.doi. org/10.1103/PhysRevLett.86.3771.

[23] Kumar, D., Nitsure, N., Bhattacharya, S. \& Ghosh, S. Granular self-organization by autotuning of friction. Proceedings of the National Academy of Sciences 112, 11443 (2015). URL http://dx.doi.org/10.1073/pnas.1500665112.

[24] Hadda, N., Nicot, F., Wan, R. \& Darve, F. Microstructural self-organization in granular materials during failure. Comptes Rendus Mécanique 343, 143 (2015). URL http://dx.doi.org/10.1016/j.crme.2014.09.009.

[25] Donev, A. Improving the density of jammed disordered packings using ellipsoids. Science 303, 990 (2004). URL http://dx.doi.org/10.1126/science.1093010.

[26] Schreck, C. F., Xu, N. \& O'Hern, C. S. A comparison of jamming behavior in systems composed of dimer- and ellipse-shaped particles. Soft Matter 6, 2960 (2010). URL http://dx.doi.org/10.1039/C001085E

[27] Haji-Akbari, A. et al. Disordered, quasicrystalline and crystalline phases of densely packed tetrahedra. Nature 462, 773 (2009). URL http://dx.doi.org/10.1038/ nature08641.

[28] Zuriguel, I., Mullin, T. \& Rotter, J. M. Effect of particle shape on the stress dip under a sandpile. Physical Review Letters 98, 028001 (2007). URL http://dx.doi. org/10.1103/PhysRevLett.98.028001.

[29] Harth, K., Trittel, T., Wegner, S. \& Stannarius, R. Free cooling of a granular gas of rodlike particles in microgravity. Physical Review Letters 120 (2018). URL http://dx.doi.org/10.1103/PhysRevLett.120.214301.

[30] Farhadi, S. \& Behringer, R. P. Dynamics of sheared ellipses and circular disks: Effects of particle shape. Physical Review Letters 112, 148301 (2014). URL http: //dx.doi.org/10.1103/PhysRevLett.112.148301 
[31] Asencio, K., Acevedo, M., Zuriguel, I. \& Maza, D. Experimental study of ordering of hard cubes by shearing. Physical Review Letters 119, 228002 (2017). URL http: //dx.doi.org/10.1103/PhysRevLett.119.228002.

[32] Kou, B. et al. Translational and rotational dynamical heterogeneities in granular systems. Physical Review Letters 121, 018002 (2018). URL http://dx.doi.org/ 10.1103/PhysRevLett.121.018002.

[33] Nadler, B., Guillard, F. \& Einav, I. Kinematic model of transient shape-induced anisotropy in dense granular flow. Physical Review Letters 120, 198003 (2018). URL http://dx.doi.org/10.1103/PhysRevLett.120.198003.

[34] Eshuis, P., van der Weele, K., van der Meer, D. \& Lohse, D. Granular leidenfrost effect: Experiment and theory of floating particle clusters. Physical Review Letters 95, 258001 (2005). URL http://dx.doi.org/10.1103/PhysRevLett.95.258001.

[35] Lei, D., Huang, J., Xu, W., Wang, W. \& Zhang, P. Deformation analysis of shear band in granular materials via a robust plane shear test and numerical simulation. Powder Technology 323, 385 (2018). URL http://dx.doi .org/10.1016/j . powtec. 2017.10 .027

[36] Chand, R., Muniandy, S. V., Wong, C. S. \& Singh, J. Discrete element method study of shear-driven granular segregation in a slowly rotating horizontal drum. Particuology 32, 89 (2017). URL http://dx.doi.org/10.1016/j.partic.2016.05.014.

[37] de Bruyn, J. R., Lewis, B. C., Shattuck, M. D. \& Swinney, H. L. Spiral patterns in oscillated granular layers. Physical Review E 63, 041305 (2001). URL http: //dx.doi.org/10.1103/PhysRevE.63.041305

[38] Jaeger, H. M., Nagel, S. R. \& Behringer, R. P. Granular solids, liquids, and gases. Reviews of Modern Physics 68, 1259 (1996). URL http://dx.doi.org/10.1103/ RevModPhys.68.1259

[39] Kadanoff, L. P. Built upon sand: Theoretical ideas inspired by granular flows. Reviews of Modern Physics 71, 435 (1999). URL http://dx.doi.org/10.1103/ RevModPhys.71.435

[40] Mukhopadhyay, A. K., Liebchen, B. \& Schmelcher, P. Simultaneous control of multispecies particle transport and segregation in driven lattices. Physical Review Letters 120, 218002 (2018). URL http://dx.doi.org/10.1103/PhysRevLett.120.218002. 
[41] Clerc, M. G. et al. Liquid-solid-like transition in quasi-one-dimensional driven granular media. Nature Physics 4, 249 (2008). URL http://dx.doi.org/10.1038/ nphys884.

[42] Vescovi, D., Berzi, D. \& di Prisco, C. Fluid-solid transition in unsteady, homogeneous, granular shear flows. Granular Matter 20 (2018). URL http://dx.doi.org/ 10.1007/s10035-018-0797-y.

[43] Gnoli, A. et al. Controlled viscosity in dense granular materials. Physical Review Letters 120, 138001 (2018). URL http://dx.doi.org/10.1103/PhysRevLett.120. 138001

[44] Capozza, R., Vanossi, A., Vezzani, A. \& Zapperi, S. Suppression of friction by mechanical vibrations. Physical Review Letters 103, 085502 (2009). URL http: //dx.doi.org/10.1103/PhysRevLett.103.085502

[45] Capozza, R., Vanossi, A., Vezzani, A. \& Zapperi, S. Triggering frictional slip by mechanical vibrations. Tribology Letters 48, 95 (2012). URL http://dx.doi.org/ 10.1007/s11249-012-0002-0.

[46] Gallas, J., Herrmann, H. \& Sokolowski, S. Molecular dynamics simulation of powder fluidization in two dimensions. Physica(Amsterdam) 189A, 437 (1992). URL https: //doi.org/10.1016/0378-4371(92)90055-U.

[47] Ristow, G., Straßburger, G. \& Rehberg, I. Phase diagram and scaling of granular materials under horizontal vibrations. Phys. Rev. Lett. 79, 833 (1997). URL https: //journals.aps.org/prl/abstract/10.1103/PhysRevLett.79.833.

[48] Götzendorfer, A., Kreft, J., Kruelle, C. \& Rehberg, I. Sublimation of a vibrated granular monolayer: Coexistence of gas and solid. Phys. Rev. Lett. 95, 135704 (2005). URL https://journals.aps.org/prl/abstract/10.1103/PhysRevLett. 95.135704.

[49] Roeller, K., Clewett, J. P. D., Bowley, R. M., Herminghaus, S. \& Swift, M. R. Liquidgas phase separation in confined vibrated dry granular matter. Physical Review Letters 107, 048002 (2011). URL http://dx.doi.org/10.1103/PhysRevLett . 107. 048002

[50] Clewett, J. P. D., Roeller, K., Bowley, R. M., Herminghaus, S. \& Swift, M. R. Emergent surface tension in vibrated, noncohesive granular media. Phys. Rev. Lett. 109, 228002 (2012). URL http://dx.doi.org/10.1103/PhysRevLett.109.228002. 
[51] Scheeres, D., Hartzell, C., Sánchez, P. \& Swift, M. Scaling forces to asteroid surfaces: The role of cohesion. Icarus 210, 968 (2010). URL http://dx.doi.org/10.1016/ j.icarus.2010.07.009.

[52] Ulrich, S. et al. Dilute wet granular particles: Nonequilibrium dynamics and structure formation. Physical Review E 80 (2009). URL http://dx.doi.org/10.1103/ PhysRevE.80.031306

[53] Ulrich, S. et al. Cooling and aggregation in wet granulates. Physical Review Letters 102, 148002 (2009). URL http://dx.doi.org/10.1103/PhysRevLett.102.148002.

[54] Fingerle, A., Roeller, K., Huang, K. \& Herminghaus, S. Phase transitions far from equilibrium in wet granular matter. New Journal of Physics 10, 053020 (2008). URL http://dx.doi.org/10.1088/1367-2630/10/5/053020.

[55] Huang, K., Roeller, K. \& Herminghaus, S. Universal and non-universal aspects of wet granular matter under vertical vibrations. The European Physical Journal Special Topics 179, 25 (2009). URL http://dx.doi.org/10.1140/epjst/e2010-01191-5.

[56] Scheel, M. et al. Morphological clues to wet granular pile stability. Nature Materials 7, 189 (2008). URL http://dx.doi.org/10.1038/nmat2117.

[57] Hager, J. S., Krug, J., Popkov, V. \& Schütz, G. M. Minimal current phase and universal boundary layers in driven diffusive systems. Physical Review E 63, 056110 (2001). URL http://dx.doi.org/10.1103/PhysRevE.63.056110.

[58] Groh, C., Rehberg, I. \& Kruelle, C. A. Particle dynamics of a cartoon dune. New Journal of Physics 12, 063025 (2010). URL http://dx.doi.org/10.1088/ 1367-2630/12/6/063025.

[59] Koos, E. \& Willenbacher, N. Capillary forces in suspension rheology. Science 331, 897 (2011). URL http://dx.doi.org/10.1126/science.1199243.

[60] Goldhirsch, I. \& Zanetti, G. Clustering instability in dissipative gases. Phys. Rev. Lett. 70, 1619 (1993). URL http://dx.doi.org/10.1103/PhysRevLett.70.1619.

[61] Brilliantov, N. V. \& Pöschel, T. Kinetic Theory of Granular Gases (Oxford University Press, 2004). URL http://dx.doi.org/10.1093/acprof:oso/ 9780198530381.001 .0001

[62] Brzinski, T. A. \& Daniels, K. E. Sounds of failure: Passive acoustic measurements of excited vibrational modes. Physical Review Letters 120, 218003 (2018). URL http://dx.doi.org/10.1103/PhysRevLett.120.218003. 
[63] O'Hern, C. S., Silbert, L. E., Liu, A. J. \& Nagel, S. R. Jamming at zero temperature and zero applied stress: The epitome of disorder. Physical Review E 68, 011306 (2003). URL http://dx.doi.org/10.1103/PhysRevE.68.011306.

[64] Herminghaus, S. Wet Granular Matter (World Scientific, 2012). URL http://dx. doi.org/10.1142/8575.

[65] Gonzalez, S., Thornton, A. \& Luding, S. Free cooling phase-diagram of hard-spheres with short- and long-range interactions. The European Physical Journal Special Topics 223, 2205 (2014). URL http://dx.doi.org/10.1140/epjst/e2014-02259-x.

[66] Murphy, E. \& Subramaniam, S. Freely cooling granular gases with short-ranged attractive potentials. Physics of Fluids 27, 043301 (2015). URL http://dx.doi. org/10.1063/1.4916674

[67] Cundall, P. A. \& Strack, O. D. L. A discrete numerical model for granular assemblies. Géotechnique 29, 47 (1979). URL http://dx.doi.org/10.1680/geot.1979.29.1. 47.

[68] Aspelmeier, T., Giese, G. \& Zippelius, A. Cooling dynamics of a dilute gas of inelastic rods: A many particle simulation. Physical Review E 57, 857 (1998). URL http://dx.doi.org/10.1103/PhysRevE.57.857

[69] Zhu, H., Zhou, Z., Yang, R. \& Yu, A. Discrete particle simulation of particulate systems: Theoretical developments. Chemical Engineering Science 62, 3378 (2007). URL http://dx.doi .org/10.1016/j.ces.2006.12.089

[70] Hertz, H. Über die berührung fester elastischer körper. Journal für die reine und angewandte Mathematik (Crelle's Journal) 92, 156 (1882). URL http://dx.doi. org/10.1515/crll.1882.92.156

[71] Mindlin, R. \& Deresiewicz, H. Elastic spheres in contact under varying oblique forces. Journal of Applied Mechanics 20, 327 (1953).

[72] Vu-Quoc, L. \& Zhang, X. An accurate and efficient tangential force-displacement model for elastic frictional contact in particle-flow simulations. Mechanics of Materials 31, 235 (1999). URL http://dx.doi .org/10.1016/S0167-6636(98)00064-7.

[73] Di Renzo, A. \& Di Maio, F. P. Comparison of contact-force models for the simulation of collisions in dem-based granular flow codes. Chemical Engineering Science 59, 525 (2004). URL http://dx.doi.org/10.1016/j.ces.2003.09.037. 
[74] Walton, O. R. Numerical simulation of inclined chute flows of monodisperse, inelastic, frictional spheres. Mechanics of Materials 16, 239 (1993). URL http://dx.doi. org/10.1016/0167-6636(93)90048-V

[75] Thornton, C. \& Yin, K. Impact of elastic spheres with and without adhesion. Powder Technology 65, 153 (1991). URL http://dx.doi.org/10.1016/0032-5910(91) 80178-L.

[76] Langston, P., Tüzün, U. \& Heyes, D. Continuous potential discrete particle simulations of stress and velocity fields in hoppers: transition from fluid to granular flow. Chemical Engineering Science 49, 1259 (1994). URL http://dx.doi.org/10.1016/ 0009-2509(94)85095-X.

[77] Rumpf, H. The strength of granules and agglomerates (Interscience, New York, 1962).

[78] Israelachvili, J. N. Intermolecular and Surface Forces (Academic Press, London, 2000).

[79] Hamaker, H. The london - van der waals attraction between spherical particles. Physica 4, 1058 (1937). URL http://dx.doi.org/10.1016/S0031-8914(37)80203-7.

[80] Lifshitz, E. M. The theory of molecular attractive forces between solids. Journal of Experimental and Theoretical Physics (Soviet) 2, 73 (1956).

[81] Krupp, H. Particle adhesion theory and experiment. Advances in Colloid and Interface Science 1, 111 (1967). URL http://dx.doi.org/10.1016/0001-8686(67) 80004-6.

[82] Yang, R. Y., Zou, R. P. \& Yu, A. B. Computer simulation of the packing of fine particles. Physical Review E 62, 3900 (2000). URL http://dx.doi.org/10.1103/ PhysRevE.62.3900.

[83] Yen, K. Z. Y. \& Chaki, T. K. A dynamic simulation of particle rearrangement in powder packings with realistic interactions. Journal of Applied Physics 71, 3164 (1992). URL http://dx.doi.org/10.1063/1.350958.

[84] Atkinson, K. E. An Introduction to Numerical Analysis (2nd Edition) (Wiley, New York, 1989).

[85] Verlet, L. Computer "experiments" on classical fluids. i. thermodynamical properties of -jones molecules. Physical Review 159, 98 (1967). URL http://dx.doi.org/10. 1103/PhysRev.159.98. 
[86] VERLET, L. Computer "experiments" on classical fluids. ii. equilibrium correlation functions. Physical Review 165, 201 (1968). URL http://dx.doi.org/10.1103/ PhysRev.165.201.

[87] Hockney, R. W. \& Eastwood, J. W. Computer simulation using particles (McGrawHill, New Yock, 1981).

[88] Knuth, D. E. The art of computer programming (2nd Edition) (Addison Wesley, 1973).

[89] Jin, Y. \& Makse, H. A. A first-order phase transition defines the random close packing of hard spheres. Physica A 389, 5362 (2010).

[90] Steinhardt, P. J., Nelson, D. R. \& Ronchetti, M. Bond-orientational order in liquids and glasses. Physical Review B 28, 784 (1983). URL http://dx.doi.org/10.1103/ PhysRevB.28.784.

[91] Rein ten Wolde, P., Ruiz-Montero, M. J. \& Frenkel, D. Numerical calculation of the rate of crystal nucleation in a lennard-jones system at moderate undercooling. The Journal of Chemical Physics 104, 9932 (1996).

[92] Filion, L., Ni, R., Frenkel, D. \& Dijkstra, M. Simulation of nucleation in almost hard-sphere colloids: The discrepancy between experiment and simulation persists. The Journal of Chemical Physics 134, 134901 (2011). URL http://dx.doi.org/ $10.1063 / 1.3572059$

[93] Panaitescu, A., Reddy, K. A. \& Kudrolli, A. Nucleation and crystal growth in sheared granular sphere packings. Physical Review Letters 108, 108001 (2012).

[94] Rietz, F., Radin, C., Swinney, H. L. \& Schröter, M. Nucleation in sheared granular matter. Physical Review Letters 120, 055701 (2018). URL http://dx.doi.org/10. 1103/PhysRevLett.120.055701

[95] Watanabe, H., Ito, N. \& Hu, C.-K. Phase diagram and universality of the lennardjones gas-liquid system. The Journal of Chemical Physics 136, 204102 (2012). URL http://dx.doi.org/10.1063/1.4720089

[96] Sack, A., Heckel, M., Kollmer, J. E., Zimber, F. \& Pöschel, T. Energy dissipation in driven granular matter in the absence of gravity. Physical Review Letters 111, 018001 (2013). 



\section{Acknowlegements}

I would never have completed this thesis without the help from so many people.

My first appreciation goes to Prof. Stephan Herminghaus and Prof. Marco G. Mazza for giving me the chance to do my $\mathrm{PhD}$ study at MPI-DS and for giving me financial support. Many thanks also go to Prof. Annette Zippelius for being one of my thesis committee members and for all the enlightening discussions during the committee meetings.

I would like to express my special gratitude to Marco, the person who always worked together with me and taught me not only about how to do physics research but also about how to efficiently communicate and cooperate with others. Thanks for never giving up on me in these years, and thanks for helping me finish this thesis in such a short time, especially during his move to England.

My thank also goes to Barbara Kutz, Monika Teuteberg, whose efficient scientific management works made my office work at the institute so easy. And thanks Thomas Eggers for all the help about the desktop and python packages.

Thanks all the people who worked and are working at the Nonequilibrium Soft Matter group. I enjoyed so much all the office time, the group meetings, group lunches and the ping-pong time after lunches we went through together. Special thanks to James, who taught and encouraged me a lot during the tough beginning times. Also special thanks to Rebekka, whose help made my life aboard much easier. And the communication with her about coding tricks and girly stories made my office time so enjoyable. So many thanks to Fabian, who kept encourage me during the hard time and came to my defence rehearsals and provided me so many productive suggestions. I also want to thank my officemate and friend Chenyu, who pushed me day and night to finish this thesis. Thanks to her for all the inspiring chatting with me which helped me going through the most frustrating time.

My memories about Göttingen would not be that wonderful without all the friends I met there. Thanks so much for all the running time, badminton time, dinner time and 
shopping time together.

So many thanks to my mom who came to Germany three times, 360 days in total, to take care of me after the delivery and then take care my son when I was focusing on my work and thesis. Thanks to my father for the understanding and supports like always. Besides, the trust from my younger brother and two younger sisters are always the driving force which pushes me to look forward.

The last but not the least, I am so grateful to my husband Shijie and my son Keqian. Shijie was doing much more than a husband. He is the person who always stood by me to go through all the joyfulness and frustration. He also helped and taught me a lot about coding and physics research. Thanks for the coming of my lovely son Keqian and all the happiness he brought to us. To be a mom makes me more and more responsible and determined. These two closest men keep making me a better person. 


\section{Erklärung}

1. Ich habe diese Arbeit selbständig und ohne unerlaubte Hilfe angefertigt und dabei nur die angegebenen Quellen benutzt.

2. Eine entsprechende Promotion wurde an keiner anderen Hochschule im In- oder Ausland beantragt; die eingereichte Dissertation oder Teile von ihr wurden nicht für ein anderes Promotionsvorhaben verwendet.

Göttingen, August 21st, 2018

Qiong Bai 